\title{
Transverse-momentum distributions in a diquark spectator model
}

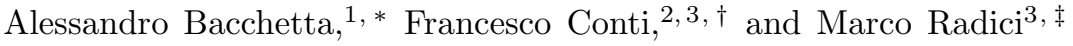 \\ ${ }^{1}$ Theory Center, Jefferson Lab, 12000 Jefferson Av., Newport News, VA 23606, USA \\ ${ }^{2}$ Dipartimento di Fisica Nucleare e Teorica, Università di Pavia, I-27100 Pavia, Italy \\ ${ }^{3}$ Istituto Nazionale di Fisica Nucleare, Sezione di Pavia, I-27100 Pavia, Italy
}

\begin{abstract}
All the leading-twist parton distribution functions are calculated in a spectator model of the nucleon, using scalar and axial-vector diquarks. Single gluon rescattering is used to generate T-odd distribution functions. Different choices for the diquark polarization states are considered, as well as a few options for the form factor at the nucleon-quark-diquark vertex. The results are listed in analytic form and interpreted in terms of light-cone wave functions. The model parameters are fixed by reproducing the phenomenological parametrization of unpolarized and helicity parton distributions at the lowest available scale. Predictions for the other parton densities are given and, whenever possible, compared with available phenomenological parametrizations.
\end{abstract}

PACS numbers: 12.39.-x, 13.60.-r, 13.88.+e

\section{INTRODUCTION}

Partonic transverse-momentum distributions (TMDs) — also called unintegrated PDFs — describe the probability to find in a hadron a parton with longitudinal momentum fraction $x$ and transverse momentum $p_{T}$ with respect to the direction of the parent hadron momentum [1]. They give a three-dimensional view of the parton distribution in momentum space, complementary to what can be obtained through generalized parton distributions [2, 3, , 4, , 5, , 6].

In the last years a lot of theoretical and experimental activity related to TMDs has taken place. Crucial steps were made in the understanding of factorization theorems involving TMDs $\left(k_{T}\right.$ factorization) 7 , 8]. Some of the properties of TMDs have been investigated from the theoretical standpoint. For instance, positivity bounds were presented in Ref. [9]. Relations among these functions in the large- $N_{c}$ limit of QCD were put forward in Ref. [10]. Their behavior at large $x$ was studied in Ref. [11], and at high transverse momentum in Ref. [12]. Last but not least, it was also demonstrated [13] that TMDs that are odd under naïve time-reversal transformations (for brevity, T-odd) can be nonzero and must be included in the complete list of leading-twist TMDs (see, e.g., Ref. [14, 15]). Their universality properties are different from the standard PDFs [16].

In the meanwhile, several azimuthal asymmetries were measured in semi-inclusive deep inelastic scattering (SIDIS) and elsewhere (see Ref. [17] and references therein), and more experimental measurements are planned. However, not much phenomenological information concerning TMDs is available as yet (see, e.g., Ref. [18] and references therein). The analysis of azimuthal spin asymmetries both in hadron-hadron collisions and in SIDIS led to the extraction of the Sivers function [19], denoted as $f_{1 T}^{\perp}$, a T-odd TMD that describes how the parton distribution is distorted by the transverse polarization of the parent hadron (see Ref. [20] for a comparison of various parametrizations). A recent attempt to extract the T-odd Boer-Mulders function, $h_{1}^{\perp}$ [21], a T-odd TMD describing the distribution of transversely polarized partons in an unpolarized hadron, was presented in Ref. [22]. All of the above studies assume a flavor-independent Gaussian distribution of the transverse momentum, although there is no compelling reason for this choice.

In this context, building a relatively simple model to compute TMDs and to allow for numerical estimates is of great importance. From the theoretical side, this can help understanding some of the essential features of TMDs, for instance their relation to the orbital angular momentum of partons (see, e.g., Refs. [13, 23, 24, 25, 26, 27, 28, 29]). From the experimental side, a model could be useful to estimate the size of observables in different processes and kinematical regimes [30, 31, 32, 33, 34, 35, 36] and to set up Monte Carlo simulations [37, 38, 39, 40, 41].

Although many model calculations of integrated PDFs are available, there are not so many for TMDs. In Ref. [42] all the leading-twist T-even functions were calculated in a spectator model with scalar and axial-vector diquarks. Recently, an analogous calculation has been performed in a light-cone quark model [43]. T-odd functions were calculated in the spectator model with scalar diquarks [13, 30, 44, 45], with scalar and vector diquarks [36, 46], in the

\footnotetext{
*Electronic address: alessandro.bacchetta@jlab.org

${ }^{\dagger}$ Electronic address: francesco.conti@pv.infn.it

${ }^{\ddagger}$ Electronic address: marco.radici@pv.infn.it
} 
MIT bag model [47, 48], in a constituent quark model [49] and in the spectator model for the pion [50]. A complete calculation of all the leading-twist TMDs in a spectator model with scalar diquarks was presented in Ref. [27].

In this work, we choose a more phenomenological approach. We consider also axial-vector diquarks (in the following often called simply vector diquarks), necessary for a realistic flavor analysis, and we further distinguish between isoscalar ( $u d$-like) and isovector ( $u u$-like) spectators. We generate the relative phase necessary to produce T-odd structures by approximating the gauge link operator with a one gluon-exchange interaction. We consider several choices of form factors at the nucleon-quark-diquark vertex and several choices for the polarization states of the diquark. All results are presented in analytic form and interpreted also in terms of overlaps of light-cone wave functions, leading to a detailed analysis of the quantum numbers of the quark-diquark system. The free parameters of the model are fixed by reproducing the phenomenological parametrization of unpolarized and longitudinally polarized parton distributions at the lowest available scale.

The paper is organized as follows. In Sec. II] the analytic form for all the leading-twist TMDs is discussed for the dipolar nucleon-diquark-quark form factor and for the light-cone choice of the diquark propagator, postponing the results for the other explored combinations to the Appendices $\mathrm{A}$ (T-even TMDs) and B] (T-odd TMDs). In Sec. III numerical results are shown and compared with phenomenological parametrizations, whenever available in the literature. In Sec. IV] some conclusions are drawn.

\section{ANALYTICAL RESULTS FOR TRANSVERSE-MOMENTUM-DEPENDENT PARTON DENSITIES}

In this section we present the fundamentals of the model and we give in analytical form the results for the light-cone wave functions (LCWFs) and the TMDs obtained in the model.

\section{A. General framework}

In the following we will make use of light-cone coordinates. We introduce the light-like vectors $n_{ \pm}$satisfying $n_{ \pm}^{2}=0, n_{+} \cdot n_{-}=1$, and we describe a generic 4 -vector $a$ as

$$
a=\left[a^{-}, a^{+}, \boldsymbol{a}_{T}\right]
$$

where $a^{ \pm}=a \cdot n_{\mp}$. We will make use of the transverse tensor $\epsilon_{T}^{i j}=\epsilon^{\mu \nu i j} n_{+\mu} n_{-\nu}$, whose only nonzero components are $\epsilon_{T}^{12}=-\epsilon_{T}^{21}=1$. We choose a frame where the hadron momentum $P$ has no transverse components, i.e.,

$$
P=\left[\frac{M^{2}}{2 P^{+}}, P^{+}, \mathbf{0}\right]
$$

The quark momentum can be written as

$$
p=\left[\frac{p^{2}+\boldsymbol{p}_{T}^{2}}{2 x P^{+}}, x P^{+}, \boldsymbol{p}_{T}\right] .
$$

In a hadronic state $|P, S\rangle$ with momentum $P$ and spin $S$, the density of quarks can be defined starting from the quark-quark correlator (see, e.g., Ref. [15])

$$
\Phi\left(x, \boldsymbol{p}_{T} ; S\right)=\left.\int \frac{d \xi^{-} d \boldsymbol{\xi}_{T}}{(2 \pi)^{3}} e^{i p \cdot \xi}\left\langle P, S\left|\bar{\psi}(0) \mathcal{U}_{[0, \xi]} \psi(\xi)\right| P, S\right\rangle\right|_{\xi^{+}=0}
$$

where

$$
U_{[0, \xi]}=\mathcal{P} e^{-i g \int_{0}^{\xi} d w \cdot A(w)}
$$

is the so-called gauge link operator, or Wilson line, connecting the two different space-time points $0, \xi$, by all possible ordered paths followed by the gluon field $A$, which couples to the quark field $\psi$ through the coupling $g$. The gauge link ensures that the matrix element of Eq. (4) is color-gauge invariant and arises from the interaction of the outgoing quark field with the spectators inside the hadron. The leading contributions of the path $[0, \xi]$ in space-time are selected by the hard process in which the parton distributions appear, thus breaking standard universality of the parton densities. For instance, in SIDIS the gauge link path in light-cone coordinates runs along

$$
[0, \xi] \equiv\left(0,0, \mathbf{0}_{T}\right) \rightarrow\left(0, \infty, \mathbf{0}_{T}\right) \rightarrow\left(0, \infty, \infty_{T}\right) \rightarrow\left(0, \infty, \boldsymbol{\xi}_{T}\right) \rightarrow\left(0, \xi^{-}, \boldsymbol{\xi}_{T}\right)
$$


while in the Drell-Yan case it runs in the opposite direction through $-\infty$. This fact leads to a sign difference in T-odd parton densities, as mentioned for the first time in Ref. [16].

Similarly to Ref. [42], we evaluate the correlator of Eq. (4) in the spectator approximation, i.e. we insert a completeness relation and at tree-level we truncate the sum over final states to a single on-shell spectator state with mass $M_{X}$, thus getting the analytic form

$$
\left.\Phi\left(x, \boldsymbol{p}_{T}, S\right) \sim \frac{1}{(2 \pi)^{3}} \frac{1}{2(1-x) P^{+}} \overline{\mathcal{M}}^{(0)}(S) \mathcal{M}^{(0)}(S)\right|_{p^{2}=\tau\left(x, \boldsymbol{p}_{T}\right)},
$$

where $p$ is the momentum of the active quark, $m$ its mass, and the on-shell condition $(P-p)^{2}=M_{X}^{2}$ for the spectator implies for the quark the off-shell condition

$$
p^{2} \equiv \tau\left(x, \boldsymbol{p}_{T}\right)=-\frac{\boldsymbol{p}_{T}^{2}+L_{X}^{2}\left(m^{2}\right)}{1-x}+m^{2}, \quad L_{X}^{2}\left(m^{2}\right)=x M_{X}^{2}+(1-x) m^{2}-x(1-x) M^{2}
$$

with $M$ the hadron mass.

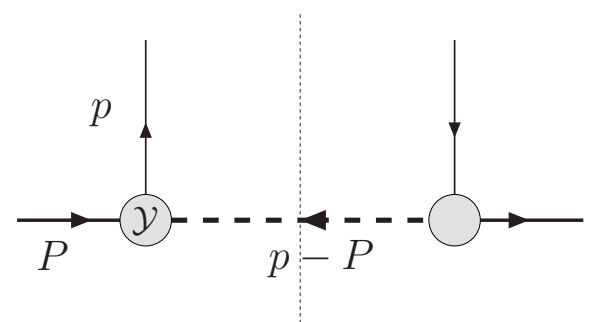

FIG. 1: Tree-level cut diagram for the calculation of T-even leading-twist parton densities. The dashed line indicates both scalar and axial-vector diquarks.

We assume the spectator to be point-like, with the quantum numbers of a diquark. Hence, the proton can couple to a quark and to a spectator diquark with spin 0 (scalar $X=s$ ) or spin 1 (axial-vector $X=a$ ), as well as with isospin 0 (isoscalar $u d$-like system) or isospin 1 (isovector $u u$-like system). Therefore, the tree-level "scattering amplitude" $\mathcal{M}^{(0)}$ is given by (see Fig. 1)

$$
\mathcal{M}^{(0)}(S)=\langle P-p|\psi(0)| P, S\rangle= \begin{cases}\frac{i}{\not p-m} \mathcal{Y}_{s} U(P, S) & \text { scalar diquark } \\ \frac{i}{\not p-m} \varepsilon_{\mu}^{*}\left(P-p, \lambda_{a}\right) \mathcal{Y}_{a}^{\mu} U(P, S) & \text { axial-vector diquark }\end{cases}
$$

and is actually a Dirac spinor because of the understood spinorial indices of the quark field $\psi$. The $\varepsilon_{\mu}\left(P-p, \lambda_{a}\right)$ is the 4 -vector polarization of the spin-1 vector diquark with momentum $P-p$ and helicity states $\lambda_{a}$. When summing over all polarizations states, several choices have been used for $d^{\mu \nu}=\sum_{\lambda_{a}} \varepsilon_{\left(\lambda_{a}\right)}^{* \mu} \varepsilon_{\left(\lambda_{a}\right)}^{\nu}$ :

$$
d^{\mu \nu}(P-p)=\left\{\begin{array}{cc}
-g^{\mu \nu}+\frac{(P-p)^{\mu} n_{-}^{\nu}+(P-p)^{\nu} n_{-}^{\mu}}{(P-p) \cdot n_{-}}-\frac{M_{a}^{2}}{\left[(P-p) \cdot n_{-}\right]^{2}} n_{-}^{\mu} n_{-}^{\nu} & \text { (see Ref. [51]) } \\
-g^{\mu \nu}+\frac{(P-p)^{\mu}(P-p)^{\nu}}{M_{a}^{2}} & \text { (see Ref. [36]) } \\
-g^{\mu \nu}+\frac{P^{\mu} P^{\nu}}{M_{a}^{2}} & \text { (see Ref. [42]) } \\
-g^{\mu \nu} & \text { (see Ref. [46]). }
\end{array}\right.
$$

The different forms for the diquark propagator correspond to different physical theories and lead to different results for the parton distribution functions. We have analyzed all of them except for the third one, which was extensively studied already in Ref. [42]. However, we think that the first one is preferable to the others. The motivation is that in the spectator model we have to take into account that the diquarks have an electric charge and can couple to the virtual photon in DIS. In other words, in this model the quarks are not the only charged partons in the proton: the diquarks are also charged partons and they have spin different from $\frac{1}{2}$. The scalar diquark couples only 
to longitudinally polarized photons and gives contribution to the structure function $F_{L}$. This leads to a violation of the Callan-Gross relation, but leaves unchanged the (leading-order) interpretation of the structure function $F_{T}$ as a charge-weighted sum of quark distribution functions. This seems the best way to reduce the phenomenological impact of the problem represented by the presence of the diquarks. For the vector diquark, we checked that the same situation occurs when only the light-cone transverse polarization states of the diquark are propagated, i.e., when the first choice of Eq. (10) for the polarization sum is used. In the other cases, the diquark would give a contribution also to the structure function $F_{T}$. On top of this, we remark that the last choice of Eq. (10) for the polarization sum introduces unphysical polarization states of the vector diquark (see discussion in next section). In conclusion, in the following we shall consider only light-cone transverse polarizations of the diquark, make only a few comments on the other choices, and leave the complete list of results in the Appendices.

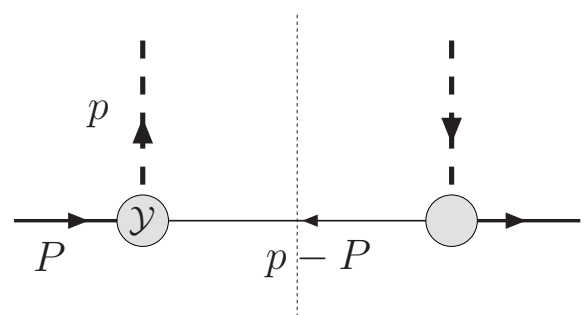

FIG. 2: Tree-level cut diagram for the calculation of T-even leading-twist parton densities for an active scalar or vector diquark (dashed line), with a spectator quark (solid line).

Equation (9) can be further elaborated by choosing the nucleon-quark-diquark vertex $\mathcal{Y}$. We choose the scalar and vector vertices to be

$$
\mathcal{Y}_{s}=i g_{s}\left(p^{2}\right) \mathbb{1}, \quad \mathcal{Y}_{a}^{\mu}=i \frac{g_{a}\left(p^{2}\right)}{\sqrt{2}} \gamma^{\mu} \gamma_{5}
$$

where $g_{X}\left(p^{2}\right)$ is a suitable form factor. Other choices are possible (see, e.g., Refs 36, 42]), but we limit ourselves to these ones, which are the simplest. For the form factor, we explored three possible choices:

$$
g_{X}\left(p^{2}\right)=\left\{\begin{array}{cl}
g_{X}^{p . l .} & \text { point-like } \\
g_{X}^{d i p} \frac{p^{2}-m^{2}}{\left|p^{2}-\Lambda_{X}^{2}\right|^{2}} & \text { dipolar, } \\
g_{X}^{\exp } e^{\left(p^{2}-m^{2}\right) / \Lambda_{X}^{2}} & \text { exponential }
\end{array}\right.
$$

where $g_{X}$ and $\Lambda_{X}$ are appropriate coupling constants and cutoffs, respectively, to be considered as free parameters of the model together with the mass of the diquark $M_{X}$. All these parameters can in principle be different for each type of diquark. Only the point-like coupling can be derived from a specific Lagrangian with protons, quarks and diquarks as fundamental degrees of freedom, and meant to effectively describe QCD in the nonperturbative regime. Since our interest here is mainly phenomenological, we prefer to introduce form factors. They smoothly suppress the influence of high $\boldsymbol{p}_{T}$ — where our theory cannot be trusted — and eliminate the logarithmic divergences arising after $\boldsymbol{p}_{T}$ integration when using a point-like coupling. For later use, we note that the dipolar form factor can be usefully rewritten, using Eq. (8), as

$$
g_{X}\left(p^{2}\right)=g_{X}^{d i p} \frac{p^{2}-m^{2}}{\left|p^{2}-\Lambda_{X}^{2}\right|^{2}}=g_{X}^{d i p} \frac{\left(p^{2}-m^{2}\right)(1-x)^{2}}{\left(\boldsymbol{p}_{T}^{2}+L_{X}^{2}\left(\Lambda_{X}^{2}\right)\right)^{2}} .
$$

In summary, we have analyzed in total nine combinations of nucleon-quark-diquark form factors and forms for the diquark propagator. As mentioned above, we will discuss analytical and numerical results involving the dipolar form factor and the first choice of Eq. (10) (transverse diquark polarizations only), listing the formulae for the other cases in the Appendices $\mathrm{A}$ and $\mathrm{B}$. To keep the notation lighter, we will denote the coupling $g_{X}^{\text {dip }}$ simply as $g_{X}$ from now on. 


\section{B. Light-cone wave functions}

A convenient way to compute parton distribution functions is by making use of light-cone wave functions (LCWFs), as done for instance in Ref. [51]. For the scalar diquark, LCWFs can be defined as

$$
\psi_{\lambda_{q}}^{\lambda_{N}}\left(x, \boldsymbol{p}_{T}\right)=\sqrt{\frac{p^{+}}{(P-p)^{+}}} \frac{\bar{u}\left(p, \lambda_{q}\right)}{p^{2}-m^{2}} \mathcal{Y}_{s} U\left(P, \lambda_{N}\right),
$$

where the indices $\lambda_{N}, \lambda_{q}$, refer to the helicity of the nucleon and of the quark, respectively, and are constrained by angular momentum conservation to the "spin sum rule" $\lambda_{N}=\lambda_{q}+L_{z}$, where $L_{z}$ is the projection of the relative orbital angular momentum between the quark and the diquark. We use the conventions of Ref. [52] (see also Ref. [27]). In standard representation, the spinors can be written as

$$
u(p,+)=\frac{1}{\sqrt{2^{3 / 2} p^{+}}}\left(\begin{array}{c}
\sqrt{2} p^{+}+m \\
p_{x}+i p_{y} \\
\sqrt{2} p^{+}-m \\
p_{x}+i p_{y}
\end{array}\right), \quad u(p,-)=\frac{1}{\sqrt{2^{3 / 2} p^{+}}}\left(\begin{array}{c}
-p_{x}+i p_{y} \\
\sqrt{2} p^{+}+m \\
p_{x}-i p_{y} \\
-\sqrt{2} p^{+}+m
\end{array}\right)
$$

and similarly for the nucleon spinors (changing $p, m$, to $P, M$, respectively). We obtain

$$
\begin{array}{rlrl}
\psi_{+}^{+}\left(x, \boldsymbol{p}_{T}\right) & =(m+x M) \phi / x & & \left(L_{z}=0\right), \\
\psi_{-}^{+}\left(x, \boldsymbol{p}_{T}\right) & =-\left(p_{x}+i p_{y}\right) \phi / x & & \left(L_{z}=+1\right), \\
\psi_{+}^{-}\left(x, \boldsymbol{p}_{T}\right) & =-\left[\psi_{-}^{+}\left(x, \boldsymbol{p}_{T}\right)\right]^{*} & & \left(L_{z}=-1\right), \\
\psi_{-}^{-}\left(x, \boldsymbol{p}_{T}\right) & =\psi_{+}^{+}\left(x, \boldsymbol{p}_{T}\right) & & \left(L_{z}=0\right), \\
\phi\left(x, \boldsymbol{p}_{T}^{2}\right) & =-\frac{g_{s}}{\sqrt{1-x}} \frac{x(1-x)}{\boldsymbol{p}_{T}^{2}+L_{s}^{2}\left(m^{2}\right)}, &
\end{array}
$$

which correspond to Eqs. (44,46) of Ref. [51].

For the vector diquark, LCWFs can be defined as

$$
\psi_{\lambda_{q} \lambda_{a}}^{\lambda_{N}}\left(x, \boldsymbol{p}_{T}\right)=\sqrt{\frac{p^{+}}{(P-p)^{+}}} \frac{\bar{u}\left(p, \lambda_{q}\right)}{p^{2}-m^{2}} \varepsilon_{\mu}^{*}\left(P-p, \lambda_{a}\right) \mathcal{Y}_{a}^{\mu} U\left(P, \lambda_{N}\right),
$$

where the index $\lambda_{a}$ refers to the helicity of the vector diquark and is constrained by $\lambda_{N}=\lambda_{q}+\lambda_{a}+L_{z}$. The light-cone transverse polarization vectors are given by [51]

$$
\begin{aligned}
& \varepsilon(P-p,+)=\left[-\frac{(P-p)_{x}+i(P-p)_{y}}{\sqrt{2}(P-p)^{+}}, 0,-\frac{1}{\sqrt{2}},-\frac{i}{\sqrt{2}}\right]=\left[\frac{p_{x}+i p_{y}}{\sqrt{2}(1-x) P^{+}}, 0,-\frac{1}{\sqrt{2}},-\frac{i}{\sqrt{2}}\right], \\
& \varepsilon(P-p,-)=\left[-\frac{p_{x}-i p_{y}}{\sqrt{2}(1-x) P^{+}}, 0, \frac{1}{\sqrt{2}},-\frac{i}{\sqrt{2}}\right] .
\end{aligned}
$$

They satisfy the usual properties ${ }^{1} \varepsilon( \pm) \cdot \varepsilon^{*}( \pm)=-1, \varepsilon( \pm) \cdot \varepsilon^{*}(\mp)=0$, and $(P-p) \cdot \varepsilon( \pm)=0$. They are consistent with the polarization sum being expressed by the first option in Eq. (10). The LCWFs become

$$
\begin{aligned}
\psi_{++}^{+}\left(x, \boldsymbol{p}_{T}\right) & =\frac{p_{x}-i p_{y}}{1-x} \phi / x & & \left(L_{z}=-1\right), \\
\psi_{+-}^{+}\left(x, \boldsymbol{p}_{T}\right) & =-x \frac{p_{x}+i p_{y}}{1-x} \phi / x & & \left(L_{z}=+1\right), \\
\psi_{-+}^{+}\left(x, \boldsymbol{p}_{T}\right) & =(m+x M) \phi / x & & \left(L_{z}=0\right), \\
\psi_{--}^{+}\left(x, \boldsymbol{p}_{T}\right) & =0 & & \left(L_{z}=+2\right), \\
\psi_{++}^{-}\left(x, \boldsymbol{p}_{T}\right) & =0 & & \left(L_{z}=-2\right),
\end{aligned}
$$

\footnotetext{
${ }^{1}$ Note that $(\boldsymbol{P}-\boldsymbol{p}) \cdot \boldsymbol{\varepsilon}( \pm) \neq 0$, since $\varepsilon( \pm)$ do not describe transverse polarization with respect to the diquark momentum.
} 


$$
\begin{array}{rlrl}
\psi_{+-}^{-}\left(x, \boldsymbol{p}_{T}\right) & =-\psi_{-+}^{+}\left(x, \boldsymbol{p}_{T}\right) & & \left(L_{z}=0\right), \\
\psi_{-+}^{-}\left(x, \boldsymbol{p}_{T}\right) & =\left[\psi_{+-}^{+}\left(x, \boldsymbol{p}_{T}\right)\right]^{*} & & \left(L_{z}=-1\right), \\
\psi_{--}^{-}\left(x, \boldsymbol{p}_{T}\right) & =\left[\psi_{++}^{+}\left(x, \boldsymbol{p}_{T}\right)\right]^{*} & & \left(L_{z}=+1\right), \\
\phi\left(x, \boldsymbol{p}_{T}^{2}\right) & =-\frac{g_{a}}{\sqrt{1-x}} \frac{x(1-x)}{\boldsymbol{p}_{T}^{2}+L_{a}^{2}\left(m^{2}\right)}, &
\end{array}
$$

and are analogous to Eqs. $(21,24)$ in Ref. [51], the differences being due to the fact that here the diquark is an axial-vector particle rather than a vector one. Note that in our model we can only have wavefunctions with at most one unit of orbital angular momentum ( $p$ wave). The LCWFs with two units of orbital angular momentum $(d$-wave), $\psi_{--}^{+}$and $\psi_{++}^{-}$, vanish.

If we add to $\varepsilon(P-p, \pm)$ also the third longitudinal polarization vector

$$
\varepsilon(P-p, 0)=\frac{1}{M_{a}}\left[\frac{p_{T}^{2}-M_{a}^{2}}{2(1-x) P^{+}},(1-x) P^{+},-p_{x},-p_{y}\right],
$$

satisfying $^{2} \varepsilon(0) \cdot \varepsilon^{*}(0)=-1, \varepsilon(0) \cdot \varepsilon^{*}( \pm)=0$, and $(P-p) \cdot \varepsilon(0)=0$, the corresponding additional LCWFs are

$$
\begin{array}{rlrl}
\psi_{+0}^{+}\left(x, \boldsymbol{p}_{T}\right) & =\frac{\boldsymbol{p}_{T}^{2}-x M_{a}^{2}-m M(1-x)^{2}}{\sqrt{2}(1-x) M_{a}} \phi / x & \left(L_{z}=0\right), \\
\psi_{-0}^{+}\left(x, \boldsymbol{p}_{T}\right)=\frac{(m+M)\left(p_{x}+i p_{y}\right)}{\sqrt{2} M_{a}} \phi / x & \left(L_{z}=+1\right), \\
\psi_{+0}^{-}\left(x, \boldsymbol{p}_{T}\right)=\left[\psi_{-0}^{+}\left(x, \boldsymbol{p}_{T}\right)\right]^{*} \phi / x & \left(L_{z}=-1\right), \\
\psi_{-0}^{-}\left(x, \boldsymbol{p}_{T}\right)=-\psi_{+0}^{+}\left(x, \boldsymbol{p}_{T}\right) \phi / x & \left(L_{z}=0\right) .
\end{array}
$$

From the above combinations we deduce, for example, that the proton with positive helicity $+\frac{1}{2}$ can be in a state with probability density proportional to $\left|\psi_{-}^{+}\right|^{2}$, where the quark has opposite helicity and $L_{z}=+1$ orbital angular momentum with respect to a scalar diquark. This configuration is relativistically enhanced with respect to $\left|\psi_{+}^{+}\right|^{2}$ with $L_{z}=0$, where proton and quark helicities are aligned; thus, it suggests a possible explanation of the proton "spin puzzle" in terms of the relativistic aspects of the motion of quarks inside hadrons [51].

For the purpose of this work, it is also important to note that a nonvanishing relative orbital angular momentum between the quark and the diquark implies that the partons do not necessarily occupy the lowest-energy available orbital (with quantum numbers $J^{P}=\frac{1}{2}^{+}$and $L_{z}=0$ ). Hence, in this version of the spectator diquark model the nucleon wave function does not show a $\mathrm{SU}(4)=\mathrm{SU}(2) \otimes \mathrm{SU}(2)$ spin-isospin symmetry, contrary to what is usually assumed [42].

Finally, we mention that the completeness relation for the last choice of the polarization sum in Eq. (10) should be written

$$
\sum_{\lambda_{a}= \pm, 0} \varepsilon^{* \mu}\left(P-p, \lambda_{a}\right) \varepsilon^{\nu}\left(P-p, \lambda_{a}\right)-\varepsilon^{* \mu}(P-p, t) \varepsilon^{\nu}(P-p, t)=-g^{\mu \nu},
$$

where the unphysical time-like polarization state $\varepsilon^{\mu}(P-p, t)=(P-p)^{\mu} / M_{a}$ appears. The associated LCWFs read

$$
\begin{aligned}
\psi_{+t}^{+}\left(x, \boldsymbol{p}_{T}\right) & =\frac{\boldsymbol{p}_{T}^{2}+x M_{a}^{2}-m M(1-x)^{2}}{\sqrt{2}(1+x) M_{a}} \phi / x & \left(L_{z}=0\right), \\
\psi_{-t}^{+}\left(x, \boldsymbol{p}_{T}\right) & =\frac{(m+M)\left(p_{x}+i p_{y}\right)}{\sqrt{2} M_{a}} \phi / x & \left(L_{z}=+1\right), \\
\psi_{+t}^{-}\left(x, \boldsymbol{p}_{T}\right) & =\left[\psi_{-t}^{+}\left(x, \boldsymbol{p}_{T}\right)\right]^{*} \phi / x & \left(L_{z}=-1\right), \\
\psi_{-t}^{-}\left(x, \boldsymbol{p}_{T}\right) & =-\psi_{+t}^{+}\left(x, \boldsymbol{p}_{T}\right) \phi / x & \left(L_{z}=0\right) .
\end{aligned}
$$

\footnotetext{
${ }^{2}$ Note that $\boldsymbol{\varepsilon}(0)$ is not parallel to $(\boldsymbol{P}-\boldsymbol{p})$ because it describes longitudinal polarization states in the light-cone.
} 


\section{T-even functions}

The simplest example of T-even parton density is the unpolarized quark distribution $f_{1}\left(x, \boldsymbol{p}_{T}\right)$, defined as

$$
\begin{aligned}
f_{1}\left(x, \boldsymbol{p}_{T}\right) & =\frac{1}{4} \operatorname{Tr}\left[\left(\Phi\left(x, \boldsymbol{p}_{T}, S\right)+\Phi\left(x, \boldsymbol{p}_{T},-S\right)\right) \gamma^{+}\right]+\text {h.c. } \\
& =\frac{1}{4} \frac{1}{(2 \pi)^{3}} \frac{1}{2(1-x) P^{+}} \operatorname{Tr}\left[\left(\overline{\mathcal{M}}^{(0)}(S) \mathcal{M}^{(0)}(S)+\overline{\mathcal{M}}^{(0)}(-S) \mathcal{M}^{(0)}(-S)\right) \gamma^{+}\right]+\text {h.c. }
\end{aligned}
$$

By inserting in $\mathcal{M}^{(0)}$ of Eq. (9) the rules (11) for the nucleon-quark-diquark vertex, the dipolar form factor of Eq. (13), and the first choice in Eq. (10) for the sum of the polarization states of the diquark (transverse polarizations only), we get

$$
\begin{aligned}
f_{1}^{q(s)}\left(x, \boldsymbol{p}_{T}\right) & =\frac{g_{s}^{2}}{(2 \pi)^{3}} \frac{\left[(m+x M)^{2}+\boldsymbol{p}_{T}^{2}\right](1-x)^{3}}{2\left[\boldsymbol{p}_{T}^{2}+L_{s}^{2}\left(\Lambda_{s}^{2}\right)\right]^{4}} \\
f_{1}^{q(a)}\left(x, \boldsymbol{p}_{T}\right) & =\frac{g_{a}^{2}}{(2 \pi)^{3}} \frac{\left[\boldsymbol{p}_{T}^{2}\left(1+x^{2}\right)+(m+x M)^{2}(1-x)^{2}\right](1-x)}{2\left[\boldsymbol{p}_{T}^{2}+L_{a}^{2}\left(\Lambda_{a}^{2}\right)\right]^{4}}
\end{aligned}
$$

The same result can be recovered through the alternative definition

$$
\begin{aligned}
& f_{1}^{q(s)}\left(x, \boldsymbol{p}_{T}^{2}\right)=\frac{1}{16 \pi^{3}} \frac{1}{2} \sum_{\lambda_{N}= \pm} \sum_{\lambda_{q}= \pm}\left|\psi_{\lambda_{q}}^{\lambda_{N}}\right|^{2}=\frac{1}{16 \pi^{3}}\left(\left|\psi_{+}^{+}\right|^{2}+\left|\psi_{-}^{+}\right|^{2}\right) \\
& f_{1}^{q(a)}\left(x, \boldsymbol{p}_{T}^{2}\right)=\frac{1}{16 \pi^{3}} \frac{1}{2} \sum_{\lambda_{N}= \pm} \sum_{\lambda_{q}= \pm} \sum_{\lambda_{a}= \pm}\left|\psi_{\lambda_{q} \lambda_{a}}^{\lambda_{N}}\right|^{2}=\frac{1}{16 \pi^{3}}\left(\left|\psi_{++}^{+}\right|^{2}+\left|\psi_{+-}^{+}\right|^{2}+\left|\psi_{-+}^{+}\right|^{2}+\left|\psi_{--}^{+}\right|^{2}\right)
\end{aligned}
$$

and replacing the results for the LCWFs using Eqs. (20) and (32) for the scalar and vector diquark, respectively.

If we use, instead, the second option of Eq. (10) for the sum over polarizations of the vector diquark (transverse and longitudinal polarizations), we obtain

$$
f_{1}^{q(a)}\left(x, \boldsymbol{p}_{T}\right)+\frac{1}{16 \pi^{3}}\left(\left|\psi_{+0}^{+}\right|^{2}+\left|\psi_{-0}^{+}\right|^{2}\right) .
$$

The complete expression is given in Eq. (A22) and corresponds to Eq. (10) of Ref. [36] with $R_{g}=0$.

Finally, the results with the last choice of Eq. (10) (transverse, longitudinal, and time-like polarizations) can be written as

$$
f_{1}^{q(a)}\left(x, \boldsymbol{p}_{T}\right)+\frac{1}{16 \pi^{3}}\left(\left|\psi_{+0}^{+}\right|^{2}+\left|\psi_{-0}^{+}\right|^{2}\right)-\frac{1}{16 \pi^{3}}\left(\left|\psi_{+t}^{+}\right|^{2}+\left|\psi_{-t}^{+}\right|^{2}\right) .
$$

Note that the contribution of the diquark time-like polarization states enters with an overall negative sign. The complete expression is given in Eq. (A26) and corresponds to Eq. (8) of Ref. [46].

Turning back to our preferred choice, i.e. the first option of Eq. (10) (light-cone transverse polarizations only), we now compute all other T-even, leading-twist TMDs. Their definition in terms of traces of the quark-quark correlator can be derived from, e.g., Eqs. (3.19) and ff. in Ref. [15]. To write them in terms of LCWFs, we need to introduce the polarization state in a generic direction $\hat{\boldsymbol{S}}_{T}=\left(\cos \phi_{S}, \sin \phi_{S}\right)$ in the transverse plane

$$
\begin{aligned}
& U(P, \uparrow)=\frac{1}{\sqrt{2}}\left(U(P,+)+e^{i \phi_{S}} U(P,-)\right), \\
& U(P, \downarrow)=\frac{1}{\sqrt{2}}\left(U(P,+)+e^{i\left(\phi_{S}+\pi\right)} U(P,-)\right) .
\end{aligned}
$$

For $\phi_{S}=0, \pi / 2$, we recover the (positive) polarizations along the $\hat{x}$ and $\hat{y}$ axis, respectively [53]. For the quark, we will use similar decompositions and use the notation $\hat{\boldsymbol{S}}_{q T}$ and $\phi_{S_{q}}$, i.e.,

$$
\begin{aligned}
& \bar{u}(p, \uparrow)=\frac{1}{\sqrt{2}}\left(\bar{u}(p,+)+e^{-i \phi_{S_{q}}} \bar{u}(p,-)\right), \\
& \bar{u}(p, \downarrow)=\frac{1}{\sqrt{2}}\left(\bar{u}(p,+)+e^{-i\left(\phi_{S_{q}}+\pi\right)} \bar{u}(p,-)\right) .
\end{aligned}
$$


With this conventions and keeping in mind that $\lambda_{X}$ is absent for the scalar diquark and $\lambda_{X}= \pm$ for the vector diquark, we can write the TMDs in the following way

$$
\begin{aligned}
g_{1 L}\left(x, \boldsymbol{p}_{T}\right) & =\frac{1}{16 \pi^{3}} \sum_{\lambda_{X}}\left(\left|\psi_{+\lambda_{X}}^{+}\right|^{2}-\left|\psi_{-\lambda_{X}}^{+}\right|^{2}\right) \\
\frac{\boldsymbol{p}_{T} \cdot \hat{\boldsymbol{S}}_{T}}{M} g_{1 T}\left(x, \boldsymbol{p}_{T}\right) & =\frac{1}{16 \pi^{3}} \sum_{\lambda_{X}}\left(\left|\psi_{+\lambda_{X}}^{\uparrow}\right|^{2}-\left|\psi_{-\lambda_{X}}^{\uparrow}\right|^{2}\right) \\
\frac{\boldsymbol{p}_{T} \cdot \hat{\boldsymbol{S}}_{q T}}{M} h_{1 L}^{\perp}\left(x, \boldsymbol{p}_{T}\right) & =\frac{1}{16 \pi^{3}} \sum_{\lambda_{X}}\left(\left|\psi_{\uparrow \lambda_{X}}^{+}\right|^{2}-\left|\psi_{\downarrow \lambda_{X}}^{+}\right|^{2}\right), \\
\hat{\boldsymbol{S}}_{T} \cdot \hat{\boldsymbol{S}}_{q_{T}} h_{1 T}\left(x, \boldsymbol{p}_{T}\right) & +\frac{\boldsymbol{p}_{T} \cdot \hat{\boldsymbol{S}}_{T}}{M} \frac{\boldsymbol{p}_{T} \cdot \hat{\boldsymbol{S}}_{q_{T}}}{M} h_{1 T}^{\perp}\left(x, \boldsymbol{p}_{T}\right)=\frac{1}{16 \pi^{3}} \sum_{\lambda_{X}}\left(\left|\psi_{\uparrow \lambda_{X}}^{\uparrow}\right|^{2}-\left|\psi_{\downarrow \lambda_{X}}^{\uparrow}\right|^{2}\right) .
\end{aligned}
$$

The above results automatically fulfill positivity bounds [9].

The explicit expressions are

$$
\begin{aligned}
g_{1 L}^{q(s)}\left(x, \boldsymbol{p}_{T}^{2}\right) & =\frac{g_{s}^{2}}{(2 \pi)^{3}} \frac{\left[(m+x M)^{2}-\boldsymbol{p}_{T}^{2}\right](1-x)^{3}}{2\left[\boldsymbol{p}_{T}^{2}+L_{s}^{2}\left(\Lambda_{s}^{2}\right)\right]^{4}} \\
g_{1 L}^{q(a)}\left(x, \boldsymbol{p}_{T}^{2}\right) & =\frac{g_{a}^{2}}{(2 \pi)^{3}} \frac{\left[\boldsymbol{p}_{T}^{2}\left(1+x^{2}\right)-(m+x M)^{2}(1-x)^{2}\right](1-x)}{2\left[\boldsymbol{p}_{T}^{2}+L_{a}^{2}\left(\Lambda_{a}^{2}\right)\right]^{4}} \\
g_{1 T}^{q(s)}\left(x, \boldsymbol{p}_{T}^{2}\right) & =\frac{g_{s}^{2}}{(2 \pi)^{3}} \frac{M(m+x M)(1-x)^{3}}{\left[\boldsymbol{p}_{T}^{2}+L_{s}^{2}\left(\Lambda_{s}^{2}\right)\right]^{4}} \\
g_{1 T}^{q(a)}\left(x, \boldsymbol{p}_{T}^{2}\right) & =\frac{g_{a}^{2}}{(2 \pi)^{3}} \frac{x(m+x M)(1-x)^{2}}{\left[\boldsymbol{p}_{T}^{2}+L_{a}^{2}\left(\Lambda_{a}^{2}\right)\right]^{4}} \\
h_{1 L}^{\perp q(s)}\left(x, \boldsymbol{p}_{T}^{2}\right) & =-\frac{g_{s}^{2}}{(2 \pi)^{3}} \frac{M(m+x M)(1-x)^{3}}{\left[\boldsymbol{p}_{T}^{2}+L_{s}^{2}\left(\Lambda_{s}^{2}\right)\right]^{4}} \\
h_{1 L}^{\perp q(a)}\left(x, \boldsymbol{p}_{T}^{2}\right) & =\frac{g_{a}^{2}}{(2 \pi)^{3}} \frac{M(m+x M)(1-x)^{2}}{\left[\boldsymbol{p}_{T}^{2}+L_{a}^{2}\left(\Lambda_{a}^{2}\right)\right]^{4}} \\
h_{1 T}^{\perp q(a)}\left(x, \boldsymbol{p}_{T}^{2}\right) & =0 . \frac{g_{s}^{2}}{(2 \pi)^{3}} \frac{M^{2}(1-x)^{3}}{\left[\boldsymbol{p}_{T}^{2}+L_{s}^{2}\left(\Lambda_{s}^{2}\right)\right]^{4}}, \\
h_{1 T}^{q(s)}\left(x, \boldsymbol{p}_{T}^{2}\right) & =\frac{g_{s}^{2}}{(2 \pi)^{3}} \frac{\left[\boldsymbol{p}_{T}^{2}+(m+x M)^{2}\right](1-x)^{3}}{2\left[\boldsymbol{p}_{T}^{2}+L_{s}^{2}\left(\Lambda_{s}^{2}\right)\right]^{4}} \\
h_{1 T}^{q(a)}\left(x, \boldsymbol{p}_{T}^{2}\right) & =-\frac{g_{a}^{2}}{(2 \pi)^{3}} \frac{\boldsymbol{p}_{T}^{2} x(1-x)}{\left[\boldsymbol{p}_{T}^{2}+L_{a}^{2}\left(\Lambda_{a}^{2}\right)\right]^{4}},
\end{aligned}
$$

From the last two formulae we deduce also the expressions for the transversity distribution:

$$
\begin{aligned}
& h_{1}^{q(s)}\left(x, \boldsymbol{p}_{T}^{2}\right)=h_{1 T}^{q(s)}\left(x, \boldsymbol{p}_{T}^{2}\right)+\frac{\boldsymbol{p}_{T}^{2}}{2 M^{2}} h_{1 T}^{\perp q(s)}\left(x, \boldsymbol{p}_{T}^{2}\right)=\frac{g_{s}^{2}}{(2 \pi)^{3}} \frac{(m+x M)^{2}(1-x)^{3}}{2\left[\boldsymbol{p}_{T}^{2}+L_{s}^{2}\left(\Lambda_{s}^{2}\right)\right]^{4}} \\
& h_{1}^{q(a)}\left(x, \boldsymbol{p}_{T}^{2}\right)=-\frac{g_{a}^{2}}{(2 \pi)^{3}} \frac{\boldsymbol{p}_{T}^{2} x(1-x)}{\left[\boldsymbol{p}_{T}^{2}+L_{a}^{2}\left(\Lambda_{a}^{2}\right)\right]^{4}} .
\end{aligned}
$$

Note that the functions $g_{1 T}$ and $h_{1 L}^{\perp}$ arise from the interference of LCWFs with $\left|L_{z}\right|=1$ and $L_{z}=0$. The function $h_{1 T}^{\perp}$ requires the interference of two LCWFs that differ by two units of $L_{z}$. This condition is necessary but not sufficient to have $h_{1 T}^{\perp} \neq 0$. In fact, the vector diquark spectator gives $h_{1 T}^{\perp}=0$ even if LCWFs with $L_{z}= \pm 1$ are present. 
Some interesting relations can be evinced from the above expressions. For example, the transversity with scalar diquark saturates the Soffer bound, while for axial-vector diquarks the relation is more involved:

$$
\begin{aligned}
& h_{1}^{q(s)}\left(x, \boldsymbol{p}_{T}^{2}\right)=\frac{1}{2}\left(f_{1}^{q(s)}\left(x, \boldsymbol{p}_{T}^{2}\right)+g_{1}^{q(s)}\left(x, \boldsymbol{p}_{T}^{2}\right)\right), \\
& h_{1}^{q(a)}\left(x, \boldsymbol{p}_{T}^{2}\right)=-\frac{x}{1+x^{2}} \frac{1}{2}\left(f_{1}^{q(a)}\left(x, \boldsymbol{p}_{T}^{2}\right)+g_{1}^{q(a)}\left(x, \boldsymbol{p}_{T}^{2}\right)\right) .
\end{aligned}
$$

When restricting to the results with scalar diquark, the $g_{1 T}$ distribution is connected to two other partners by the relations

$$
g_{1 T}^{q(s)}\left(x, \boldsymbol{p}_{T}^{2}\right)=-h_{1 L}^{\perp q(s)}\left(x, \boldsymbol{p}_{T}^{2}\right), \quad \quad g_{1 T}^{q(s)}\left(x, \boldsymbol{p}_{T}^{2}\right)=\frac{2 M}{m+x M} h_{1}^{q(s)}\left(x, \boldsymbol{p}_{T}^{2}\right),
$$

while for axial-vector diquarks we have

$$
g_{1 T}^{q(a)}\left(x, \boldsymbol{p}_{T}^{2}\right)=x h_{1 L}^{\perp q(a)}\left(x, \boldsymbol{p}_{T}^{2}\right) .
$$

This relation is however different when considering spectator diquarks with more degrees of freedom (see App. A). Our results seem to indicate that no general relation exists between $g_{1 T}$ and $h_{1 L}^{\perp}$, contrary to what is proposed in Ref. [43]. The reason is connected to the difference between LCWFs with $L_{z}=1$ and $L_{z}=-1$, as in Eqs. (24) and (25). We also observe that in the vector-diquark case $g_{1 L}-h_{1}$ and $h_{1 T}^{\perp}$ are not simply related through the relation suggested in Ref. [54]. We are led to conclude that such a relation is not general.

The $\boldsymbol{p}_{T}$-integrated results are

$$
\begin{aligned}
& f_{1}^{q(s)}(x)=\frac{g_{s}^{2}}{(2 \pi)^{2}} \frac{\left[2(m+x M)^{2}+L_{s}^{2}\left(\Lambda_{s}^{2}\right)\right](1-x)^{3}}{24 L_{s}^{6}\left(\Lambda_{s}^{2}\right)} \\
& f_{1}^{q(a)}(x)=\frac{g_{a}^{2}}{(2 \pi)^{2}} \frac{\left[2(m+x M)^{2}(1-x)^{2}+\left(1+x^{2}\right) L_{a}^{2}\left(\Lambda_{a}^{2}\right)\right](1-x)}{24 L_{a}^{6}\left(\Lambda_{a}^{2}\right)} \\
& g_{1}^{q(s)}(x)=\frac{g_{s}^{2}}{(2 \pi)^{2}} \frac{\left[2(m+x M)^{2}-L_{s}^{2}\left(\Lambda_{s}^{2}\right)\right](1-x)^{3}}{24 L_{s}^{6}\left(\Lambda_{s}^{2}\right)} \\
& g_{1}^{q(a)}(x)=-\frac{g_{a}^{2}}{(2 \pi)^{2}} \frac{\left[2(m+x M)^{2}(1-x)^{2}-\left(1+x^{2}\right) L_{a}^{2}\left(\Lambda_{a}^{2}\right)\right](1-x)}{24 L_{a}^{6}\left(\Lambda_{a}^{2}\right)} \\
& h_{1}^{q(s)}(x)=\frac{g_{s}^{2}}{(2 \pi)^{2}} \frac{(m+x M)^{2}(1-x)^{3}}{12 L_{s}^{6}\left(\Lambda_{s}^{2}\right)} \\
& h_{1}^{q(a)}(x)=-\frac{g_{a}^{2}}{(2 \pi)^{2}} \frac{x(1-x)}{12 L_{a}^{4}\left(\Lambda_{a}^{2}\right)} .
\end{aligned}
$$

\section{T-odd functions}

The two leading-twist T-odd structures are the Sivers and Boer-Mulders distributions. They are defined as

$$
\begin{aligned}
\frac{\varepsilon_{T}^{i j} p_{T i} S_{T j}}{M} f_{1 T}^{\perp}\left(x, \boldsymbol{p}_{T}^{2}\right) & =-\frac{1}{4} \operatorname{Tr}\left[\left(\Phi\left(x, \boldsymbol{p}_{T}, S\right)-\Phi\left(x, \boldsymbol{p}_{T},-S\right)\right) \gamma^{+}\right]+\text {h.c. }, \\
\frac{\varepsilon_{T}^{i j} p_{T j}}{M} h_{1}^{\perp}\left(x, \boldsymbol{p}_{T}^{2}\right) & =\frac{1}{4} \operatorname{Tr}\left[\left(\Phi\left(x, \boldsymbol{p}_{T}, S\right)+\Phi\left(x, \boldsymbol{p}_{T},-S\right)\right) i \sigma^{i+} \gamma_{5}\right]+\text { h.c. } .
\end{aligned}
$$

At tree level, these expressions vanish because there is no residual interaction between the active quark and the spectators; equivalently, there is no interference between two competing channels producing the complex amplitude whose imaginary part gives the T-odd contribution. We can generate such structures by considering the interference between the tree-level scattering amplitude and the single-gluon-exchange scattering amplitude in eikonal approximation, as shown in Fig. 3 (the Hermitean conjugate partner must also be considered). This corresponds just to the leading-twist one-gluon-exchange approximation of the gauge link operator of Eq. (5) [55]. 


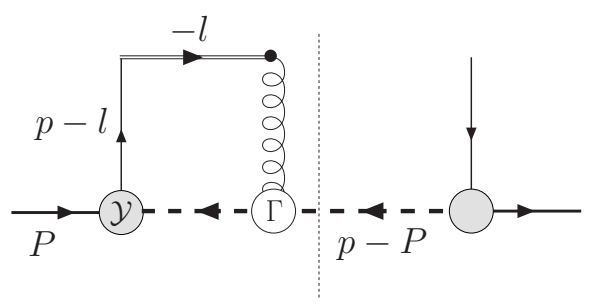

FIG. 3: Interference between the one-gluon exchange diagram in eikonal approximation and the tree level diagram in the spectator model. The Hermitean conjugate diagram is not shown.

For the moment, we use Abelian gluons. The QCD color structure will be recovered at the end. The Feynman rules to be used for the eikonal vertex and propagator are [1, 56]

$$
\mathcal{g}_{\rho}=-i e_{c} n_{-}^{\rho}, \quad \Longrightarrow=\frac{i}{-l^{+}+i \epsilon},
$$

where $e_{c}$ is the color charge of the quark and the sign of $i \epsilon$ for the eikonal line corresponds to the gauge link of SIDIS. In cut diagrams one must take the complex conjugate of these expressions for vertices and propagators on the right of the final-state cut.

The explicit form of the contribution $\Phi^{(1)}$ to the correlation function corresponding to Fig. 3 is

$$
\left.\Phi^{(1)}\left(x, \boldsymbol{p}_{T}, S\right) \sim \frac{1}{(2 \pi)^{3}} \frac{1}{2(1-x) P^{+}}\left(\overline{\mathcal{M}}^{(0)}(S) \mathcal{M}^{(1)}(S)+\overline{\mathcal{M}}^{(1)}(S) \mathcal{M}^{(0)}(S)\right)\right|_{p^{2}=\tau\left(x, \boldsymbol{p}_{T}\right)},
$$

where $\tau\left(x, \boldsymbol{p}_{T}\right)$ is defined in Eq. (8) and

$$
\mathcal{M}^{(1)}(S)= \begin{cases}-\int \frac{d^{4} l}{(2 \pi)^{4}} \frac{i e_{c} \Gamma_{s \rho} n_{-}^{\rho}(\not p-\not p+m) \mathcal{Y}_{s} U(P, S)}{\left(D_{1}+i \varepsilon\right)\left(D_{2}-i \varepsilon\right)\left(D_{3}+i \varepsilon\right)\left(D_{4}+i \varepsilon\right)} & \text { scalar diquark, } \\ -\int \frac{d^{4} l}{(2 \pi)^{4}} \frac{i e_{c} \varepsilon_{\sigma}^{*}\left(P-p, \lambda_{a}\right) \Gamma_{a \rho}^{\nu \sigma} n_{-}^{\rho}(\not p-\not p+m) d_{\mu \nu}(p-l-P) \mathcal{Y}_{a}^{\mu} U(P, S)}{\left(D_{1}+i \varepsilon\right)\left(D_{2}-i \varepsilon\right)\left(D_{3}+i \varepsilon\right)\left(D_{4}+i \varepsilon\right)} & \text { axial-vector diquark, }\end{cases}
$$

where for convenience we have introduced the notation

$$
\begin{aligned}
& D_{1}=l^{2}-m_{g}^{2}, \\
& D_{2}=l^{+}, \\
& D_{3}=(p-l)^{2}-m^{2}, \\
& D_{4}=(P-p+l)^{2}-M_{X}^{2} .
\end{aligned}
$$

In order to explicitly calculate $\mathcal{M}^{(1)}$, we need to model the gluon vertex with the scalar $\left(\Gamma_{s}\right)$ and axial vector $\left(\Gamma_{a}\right)$ diquark in Fig. 3.

$$
\begin{aligned}
\Gamma_{s}^{\rho} & =i e_{c}(2 P-2 p+l)^{\rho} \\
\Gamma_{a \rho}^{\nu \sigma} & =-i e_{c}\left[(2 P-2 p+l)_{\rho} g^{\nu \sigma}-\left(P-p+\left(1+\kappa_{a}\right) l\right)^{\sigma} g_{\rho}^{\nu}-\left(P-p-\kappa_{a} l\right)^{\nu} g_{\rho}^{\sigma}\right],
\end{aligned}
$$

where $e_{c}$ is the diquark color charge, which is the same for scalar and vector ones and identical to that of the quark; $\kappa_{a}$ is the diquark anomalous chromomagnetic moment. The structure of the vector diquark-gluon vertex resembles the one for the coupling between the photon and a spin-1 particle (see, e.g., Ref. [57]); for $\kappa_{a}=1$ the standard point-like photon- $W$ coupling is recovered (see, e.g., Ref. [58]).

The Sivers and Boer-Mulders functions can then be computed as

$$
\begin{aligned}
\frac{\varepsilon_{T}^{i j} p_{T i} S_{T j}}{M} f_{1 T}^{\perp}\left(x, \boldsymbol{p}_{T}^{2}\right) & =-\frac{1}{4} \frac{1}{(2 \pi)^{3}} \frac{1}{2(1-x) P^{+}} \operatorname{Tr}\left[\left(\mathcal{M}^{(1)}(S) \overline{\mathcal{M}}^{(0)}(S)-\mathcal{M}^{(1)}(-S) \overline{\mathcal{M}}^{(0)}(-S)\right) \gamma^{+}\right]+\text {h.c. }, \\
\frac{\varepsilon_{T}^{i j} p_{T j}}{M} h_{1}^{\perp}\left(x, \boldsymbol{p}_{T}^{2}\right) & =\frac{1}{4} \frac{1}{(2 \pi)^{3}} \frac{1}{2(1-x) P^{+}} \operatorname{Tr}\left[\left(\mathcal{M}^{(1)}(S) \overline{\mathcal{M}}^{(0)}(S)+\mathcal{M}^{(1)}(-S) \overline{\mathcal{M}}^{(0)}(-S)\right) i \sigma^{i+} \gamma_{5}\right]+\text { h.c. } .
\end{aligned}
$$


Again, results have been produced for the three different choices of both Eq. (12) for the form factors at the nucleon-quark-diquark vertex, as well as of the axial-vector diquark propagator on each side of the diquark-gluon vertex in Fig. 3. Consistently with the case of T-even parton densities, here we show the results for the dipolar form factor of Eq. (13) and for the light-cone transverse polarizations of the vector diquark, i.e. the first choice in Eq. (10), the other combinations being listed in App. B. Combining the rules (11) with the (86) ones, we can rewrite Eq. (87) and (88) as

$$
\begin{aligned}
f_{1 T}^{\perp q(s)}\left(x, \boldsymbol{p}_{T}^{2}\right) & =-\frac{g_{s}}{4} \frac{1}{(2 \pi)^{3}} \frac{M e_{c}^{2}}{2(1-x) P^{+}} \frac{(1-x)^{2}}{\left[\boldsymbol{p}_{T}^{2}+L_{s}^{2}\left(\Lambda_{s}^{2}\right)\right]^{2}} 2 \operatorname{Im} J_{1}^{s} \\
f_{1 T}^{\perp q(a)}\left(x, \boldsymbol{p}_{T}^{2}\right) & =\frac{g_{a}}{4} \frac{1}{(2 \pi)^{3}} \frac{M e_{c}^{2}}{4(1-x) P^{+}} \frac{(1-x)^{2}}{\left[\boldsymbol{p}_{T}^{2}+L_{a}^{2}\left(\Lambda_{a}^{2}\right)\right]^{2}} 2 \operatorname{Im} J_{1}^{a} \\
h_{1}^{\perp q(s)}\left(x, \boldsymbol{p}_{T}^{2}\right) & =f_{1 T}^{\perp q(s)}\left(x, \boldsymbol{p}_{T}^{2}\right) \\
h_{1}^{\perp q(a)}\left(x, \boldsymbol{p}_{T}^{2}\right) & =-\frac{1}{x} f_{1 T}^{\perp q(a)}\left(x, \boldsymbol{p}_{T}^{2}\right) .
\end{aligned}
$$

Note that for scalar diquarks the spectator model gives the same result for the Sivers and the Boer-Mulders functions, independent of the choice of the nucleon-quark-diquark form factor (see App. B).

In Eqs. (89) and ff., the expressions $J_{1}$ contain the integral over the loop momentum, the denominators $D_{1,2,3,4}$ defined in Eq. (85), and the evaluation of the trace of the projected amplitude. For instance (see App. B 1)

$$
J_{1}^{s}=\int \frac{d^{4} l}{(2 \pi)^{4}} \frac{g_{s}\left((p-l)^{2}\right)}{\left(D_{1}+i \varepsilon\right)\left(D_{2}-i \varepsilon\right)\left(D_{3}+i \varepsilon\right)\left(D_{4}+i \varepsilon\right)} 4 i\left(l^{+}+2(1-x) P^{+}\right)\left(l^{+} M-P^{+}(m+x M) \frac{\boldsymbol{l}_{T} \cdot \boldsymbol{p}_{T}}{\boldsymbol{p}_{T}^{2}}\right) .
$$

To calculate its imaginary part, it is sufficient to make the replacements

$$
\frac{1}{D_{2}-i \varepsilon} \rightarrow 2 \pi i \delta\left(D_{2}\right), \quad \frac{1}{D_{4}+i \varepsilon} \rightarrow-2 \pi i \delta\left(D_{4}\right)
$$

which corresponds to applying the Cutkosky rules [59], cutting the diquark propagator $\left(D_{4}\right)$ and the eikonalized quark one $\left(D_{2}\right)$. We then get

$$
\begin{aligned}
2 \operatorname{Im} J_{1}^{s} & =\int \frac{d^{4} l}{(2 \pi)^{4}} \frac{g_{s}\left((p-l)^{2}\right)}{D_{1} D_{3}} 4\left(l^{+}+2(1-x) P^{+}\right)\left(l^{+} M-P^{+}(m+x M) \frac{\boldsymbol{l}_{T} \cdot \boldsymbol{p}_{T}}{\boldsymbol{p}_{T}^{2}}\right)(2 \pi i) \delta\left(D_{2}\right)(-2 \pi i) \delta\left(D_{4}\right) \\
& =-4 P^{+}(m+x M)(1-x) g_{s} \mathcal{I}_{1} .
\end{aligned}
$$

The calculation of $\mathcal{I}_{1}$ depends on the form factor used. Their calculation can be found in App. C. For the case of the dipolar form factor we obtain

$$
-4 P^{+}(m+x M)(1-x) g_{s} \mathcal{I}_{1}^{d i p}=g_{s} \frac{P^{+}(m+x M)(1-x)^{2}}{\pi L_{s}^{2}\left(\Lambda_{s}^{2}\right)\left[\boldsymbol{p}_{T}^{2}+L_{s}^{2}\left(\Lambda_{s}^{2}\right)\right]} .
$$

If the T-odd structures were deduced from the Drell-Yan amplitude, the $\mathcal{M}^{(1)}$ of Eq. (84) would involve a $\left(l^{+}+i \varepsilon\right)$ propagator, leading to the opposite sign in the cutting rule for $D_{2}$. In the spectator model, this is the origin of the predicted sign change for $f_{1 T}^{\perp}$ and $h_{1}^{\perp}$ when extracting them in Drell-Yan spin asymmetries rather than in SIDIS ones [16]. Analogously to Eq. (95), we obtain

$$
2 \operatorname{Im} J_{1}^{a}=-8 P^{+} x(m+x M) g_{a} \mathcal{I}_{1}^{d i p}=g_{a} \frac{2 P^{+} x(1-x)(m+x M)}{\pi L_{a}^{2}\left(\Lambda_{a}^{2}\right)\left[\boldsymbol{p}_{T}^{2}+L_{a}^{2}\left(\Lambda_{a}^{2}\right)\right]} .
$$

By inserting these results in the model expressions of Eqs. (89) to (92), we come to the final form of the Sivers and Boer-Mulders functions with scalar and axial vector diquarks: 


$$
\begin{aligned}
& f_{1 T}^{\perp q(s)}\left(x, \boldsymbol{p}_{T}^{2}\right)=-\frac{g_{s}^{2}}{4} \frac{M e_{c}^{2}}{(2 \pi)^{4}} \frac{(1-x)^{3}(m+x M)}{L_{s}^{2}\left(\Lambda_{s}^{2}\right)\left[\boldsymbol{p}_{T}^{2}+L_{s}^{2}\left(\Lambda_{s}^{2}\right)\right]^{3}} \\
& f_{1 T}^{\perp q(a)}\left(x, \boldsymbol{p}_{T}^{2}\right)=\frac{g_{a}^{2}}{4} \frac{M e_{c}^{2}}{(2 \pi)^{4}} \frac{(1-x)^{2} x(m+x M)}{L_{a}^{2}\left(\Lambda_{a}^{2}\right)\left[\boldsymbol{p}_{T}^{2}+L_{a}^{2}\left(\Lambda_{a}^{2}\right)\right]^{3}} \\
& h_{1}^{\perp q(s)}\left(x, \boldsymbol{p}_{T}^{2}\right)=f_{1 T}^{\perp q(s)}\left(x, \boldsymbol{p}_{T}^{2}\right) \\
& h_{1}^{\perp q(a)}\left(x, \boldsymbol{p}_{T}^{2}\right)=-\frac{1}{x} f_{1 T}^{\perp q(a)}\left(x, \boldsymbol{p}_{T}^{2}\right) .
\end{aligned}
$$

To connect the "Abelian" version of the gluon interaction to the QCD color interaction we shall apply the replacement 13

$$
e_{c}^{2} \rightarrow 4 \pi C_{F} \alpha_{s} .
$$

The Sivers and Boer-Mulders functions obtained in our model behave as $1 / \boldsymbol{p}_{T}^{6}$ at high $\boldsymbol{p}_{T}^{2}$, similarly to the $f_{1}$ in Eq. (44). As observed also in Ref. [60], this leads to a breaking of the positivity bounds [9] for sufficiently high values of $\boldsymbol{p}_{T}^{2}$. This problem is due to the fact that the T-odd functions have been calculated at order $\alpha_{S}^{1}$, while the T-even functions at order $\alpha_{S}^{0}$. At high $\boldsymbol{p}_{T}^{2}$, QCD radiative corrections generate a $1 / \boldsymbol{p}_{T}^{2}$ tail for $f_{1}$ and a $1 / \boldsymbol{p}_{T}^{4}$ tail for $f_{1 T}^{\perp}[12]$. Our model is supposed to be valid for $\boldsymbol{p}_{T}^{2} \sim M^{2}$ and for reasonable choices of the parameters no problems with positivity occurr in this region.

Often the following transverse-momentum moments of the Sivers and Boer-Mulders functions are used:

$$
\begin{aligned}
f_{1 T}^{\perp(1)}(x) & =\int d \boldsymbol{p}_{T} \frac{\boldsymbol{p}_{T}^{2}}{2 M^{2}} f_{1 T}^{\perp}\left(x, \boldsymbol{p}_{T}^{2}\right) \\
f_{1 T}^{\perp(1 / 2)}(x) & =\int d \boldsymbol{p}_{T} \frac{\left|\boldsymbol{p}_{T}\right|}{2 M} f_{1 T}^{\perp}\left(x, \boldsymbol{p}_{T}^{2}\right) .
\end{aligned}
$$

In our model, they turn out to be

$$
\begin{aligned}
& f_{1 T}^{\perp q(s)(1)}(x)=-\frac{g_{s}^{2}}{32} \frac{e_{c}^{2}}{(2 \pi)^{3} M} \frac{(m+x M)(1-x)^{3}}{\left[L_{s}^{2}\left(\Lambda_{s}^{2}\right)\right]^{2}} \\
& f_{1 T}^{\perp q(a)(1)}(x)=\frac{g_{a}^{2}}{32} \frac{e_{c}^{2}}{(2 \pi)^{3} M} \frac{x(m+x M)(1-x)^{2}}{\left[L_{a}^{2}\left(\Lambda_{a}^{2}\right)\right]^{2}}, \\
& h_{1}^{\perp q(s)(1)}(x)=f_{1 T}^{\perp q(s)(1)}(x) \\
& h_{1}^{\perp q(a)(1)}(x)=-\frac{1}{x} f_{1 T}^{\perp q(a)(1)}(x) . \\
& f_{1 T}^{\perp q(s)(1 / 2)}(x)=-\frac{g_{s}^{2}}{256} \frac{e_{c}^{2}}{(2 \pi)^{2}} \frac{(m+x M)(1-x)^{3}}{\left[L_{s}^{2}\left(\Lambda_{s}^{2}\right)\right]^{5 / 2}}, \\
& f_{1 T}^{\perp q(a)(1 / 2)}(x)=\frac{g_{a}^{2}}{256} \frac{e_{c}^{2}}{(2 \pi)^{2}} \frac{x(m+x M)(1-x)^{2}}{\left[L_{a}^{2}\left(\Lambda_{a}^{2}\right)\right]^{5 / 2}}, \\
& h_{1}^{\perp q(s)(1 / 2)}(x)=f_{1 T}^{\perp q(s)(1 / 2)}(x) \\
& h_{1}^{\perp q(a)(1 / 2)}(x)=-\frac{1}{x} f_{1 T}^{\perp q(a)(1 / 2)}(x) .
\end{aligned}
$$

\section{E. T-odd functions: overlap representation}

As already mentioned above, T-odd leading-twist parton distributions arise from the interference of two channels leading to the same final state; for the case considered here (and depicted in Fig. 3), the two channels are given by 
the tree-level and the single-gluon-exchange scattering amplitudes, respectively. In Ref. [61], it was suggested that T-odd parton densities can also be represented by overlaps of LCWFs, as for their T-even partners, provided that a suitable operator is included to describe the final-state interactions (FSI) produced by the gluon rescattering. So far, this representation was fully developed in a spectator model only for the Sivers function with scalar diquarks [24, 26]. Here, we generalize it to the case of axial-vector diquarks, as well as to the Boer-Mulders function. In this way, all leading-twist (T-even and T-odd) parton densities can be given by overlaps of LCWFs consistently within the model, contrary to the statement of Ref. [27].

Following Ref. [26], for a nucleon transverse polarization state described by Eqs. (50) and (51) along a generic direction $\hat{\boldsymbol{S}}_{T}=\left(\cos \phi_{S}, \sin \phi_{S}\right)$, and for an analogous quark state described by Eqs. (52) and (53) along $\hat{\boldsymbol{S}}_{q T}=$ $\left(\cos \phi_{S_{q}}, \sin \phi_{S_{q}}\right)$, we can rewrite the Sivers (80) and Boer-Mulders (81) functions according to the Trento Conventions [62] (keeping in mind that $\lambda_{X}$ is absent for the scalar diquark and $\lambda_{X}= \pm$ for the vector diquark) as

$$
\begin{aligned}
\frac{2\left(\hat{\boldsymbol{S}}_{T} \times \boldsymbol{p}_{T}\right) \cdot \hat{\boldsymbol{P}}}{M} f_{1 T}^{\perp}\left(x, \boldsymbol{p}_{T}^{2}\right)= & \int \frac{d \boldsymbol{p}_{T}^{\prime}}{16 \pi^{3}} G\left(x, \boldsymbol{p}_{T}, \boldsymbol{p}_{T}^{\prime}\right) \\
& \sum_{\lambda_{q}, \lambda_{X}}\left[\psi_{\lambda_{q} \lambda_{X}}^{\uparrow *}\left(x, \boldsymbol{p}_{T}\right) \psi_{\lambda_{q} \lambda_{X}}^{\uparrow}\left(x, \boldsymbol{p}_{T}^{\prime}\right)-\psi_{\lambda_{q} \lambda_{X}}^{\downarrow *}\left(x, \boldsymbol{p}_{T}\right) \psi_{\lambda_{q} \lambda_{X}}^{\downarrow}\left(x, \boldsymbol{p}_{T}^{\prime}\right)\right]+\text { h.c. }, \\
\frac{\left(\hat{\boldsymbol{S}}_{q_{T}} \times \boldsymbol{p}_{T}\right) \cdot \hat{\boldsymbol{P}}}{M} h_{1}^{\perp}\left(x, \boldsymbol{p}_{T}^{2}\right)= & \int \frac{d \boldsymbol{p}_{T}^{\prime}}{16 \pi^{3}} G\left(x, \boldsymbol{p}_{T}, \boldsymbol{p}_{T}^{\prime}\right) \\
& \frac{1}{2} \sum_{\lambda_{N}, \lambda_{X}}\left[\psi_{\uparrow \lambda_{X}}^{\lambda_{N} *}\left(x, \boldsymbol{p}_{T}\right) \psi_{\uparrow \lambda_{X}}^{\lambda_{N}}\left(x, \boldsymbol{p}_{T}^{\prime}\right)-\psi_{\downarrow \lambda_{X}}^{\lambda_{N} *}\left(x, \boldsymbol{p}_{T}\right) \psi_{\downarrow \lambda_{X}}^{\lambda_{N}}\left(x, \boldsymbol{p}_{T}^{\prime}\right)\right]+\text { h.c. . }
\end{aligned}
$$

The above equations should be considered as assumptions, since it is not known a priori if the FSI operator $G\left(x, \boldsymbol{p}_{T}, \boldsymbol{p}_{T}^{\prime}\right)$ can be isolated and is the same for all functions and all types of diquarks. In our model, it turns out to be actually the same in all cases. In order to determine it, we must insert here above the expressions for the LCWFs of Sec. II B, and compare the results with the ones from Eqs. (89) to (92), after replacing $\operatorname{Im} J_{1}^{s}$, $\operatorname{Im} J_{1}^{a}$ with Eqs. (95,97), respectively, while keeping the definition of $\mathcal{I}_{1}^{\text {dip }}$ (see App. $\mathrm{C}$ ). For the scalar diquark case, for example, we get

$$
f_{1 T}^{\perp q(s)}\left(x, \boldsymbol{p}_{T}^{2}\right)=\frac{g_{s}^{2}}{8 \pi^{3}} \frac{M(1-x)^{3}(m+x M)}{\left[\boldsymbol{p}_{T}^{2}+L_{s}^{2}\left(\Lambda_{s}^{2}\right)\right]^{2}} \int d \boldsymbol{p}_{T}^{\prime} \frac{\operatorname{Im} G\left(x, \boldsymbol{p}_{T}, \boldsymbol{p}_{T}^{\prime}\right)}{\left[\boldsymbol{p}_{T}^{\prime 2}+L_{s}^{2}\left(\Lambda_{s}^{2}\right)\right]^{2}} \frac{\left(\boldsymbol{p}_{T}-\boldsymbol{p}_{T}^{\prime}\right) \cdot \boldsymbol{p}_{T}}{\boldsymbol{p}_{T}^{2}} .
$$

The above expression is identical to Eq. (89), after inserting Eq. (96) and the definition (C3) of $\mathcal{I}_{1}^{\text {dip }}$ (with the harmless substitution $\boldsymbol{l}_{T}^{\prime} \leftrightarrow-\boldsymbol{l}_{T}^{\prime}$ ), provided that

$$
\operatorname{Im} G\left(x, \boldsymbol{p}_{T}, \boldsymbol{p}_{T}^{\prime}\right)=-\frac{e_{c}^{2}}{2(2 \pi)^{2}} \frac{1}{\left(\boldsymbol{p}_{T}-\boldsymbol{p}_{T}^{\prime}\right)^{2}}=-\frac{C_{F} \alpha_{s}}{2 \pi} \frac{1}{\left(\boldsymbol{p}_{T}-\boldsymbol{p}_{T}^{\prime}\right)^{2}},
$$

in agreement with the expression of Ref. [26]. Following similar steps, we recover the same result (115) also for the Sivers function with axial-vector diquarks, and for the Boer-Mulders function as well. The FSI operator $G\left(x, \boldsymbol{p}_{T}, \boldsymbol{p}_{T}^{\prime}\right)$ is indeed universal and describes a rescattering via one gluon-exchange, which corresponds to the expansion at first order of the gauge link operator of Eq. (5). Note that in other versions of the model (see App. B) the FSI cannot be as simple as Eq. (115), since we observe also a dependence on the vector diquark anomalous chromomagnetic moment $\kappa_{a}$, which is absent in the above equation. This does not imply that the FSI operator is not universal, but simply that it could have additional parts that are not interacting with scalar diquarks and transversely polarized vector diquarks.

We close this section by observing that in our model we can generalize the relation between the first $\boldsymbol{p}_{T}$-moment of the Sivers function and the nucleon anomalous magnetic moment $\kappa$, suggested in Ref. [26] in the simple scalar diquark picture. In fact, we define $\kappa$ in terms of the Dirac form factor using the overlap representation for the nucleon matrix element of the spin-flip electromagnetic current operator [51]:

$$
\begin{aligned}
\kappa & =\frac{e}{2 M} F_{2}(0) \\
& =-\left.\frac{1}{q_{x}-i q_{y}} \sum_{k, n, \lambda_{n}} e_{n} \int \frac{d \boldsymbol{p}_{T} d x}{16 \pi^{3}} \Psi_{k}^{+*}\left(x, \boldsymbol{p}_{T}^{\prime}, \lambda_{n}\right) \Psi_{k}^{-}\left(x, \boldsymbol{p}_{T}, \lambda_{n}\right)\right|_{\boldsymbol{p}_{T}^{\prime}=\boldsymbol{p}_{T}},
\end{aligned}
$$

where the sum runs upon the number of Fock states $k$, the number of constituents $n$ in each state $k$, and their helicities $\lambda_{n}$. Since in the diquark model of the nucleon initially at rest $\left(\boldsymbol{P}_{T}=0\right)$ there is only one Fock state with 
two constituents, and the kinematics of the diquark is constrained to the one of the valence quark, the wave functions $\Psi$ reduce to the usual LCWF [26]. The momentum conservation for the struck quark reads $\boldsymbol{p}_{T}^{\prime}=\boldsymbol{p}_{T}+(1-x) \boldsymbol{q}_{T}$. Distinguishing between $\kappa^{q(s)}$ and $\kappa^{q(a)}$ for scalar and axial-vector diquarks, respectively, Eq. (116) becomes

$$
\begin{aligned}
\kappa^{q(s)} & =-\left.\frac{1}{q_{x}-i q_{y}} \int \frac{d \boldsymbol{p}_{T} d x}{16 \pi^{3}} \sum_{\lambda_{q}}\left[\psi_{\lambda_{q}}^{+*}\left(x, \boldsymbol{p}_{T}^{\prime}\right) \psi_{\lambda_{q}}^{-}\left(x, \boldsymbol{p}_{T}\right)\right]\right|_{\boldsymbol{p}_{T}^{\prime}=\boldsymbol{p}_{T}} \\
& =\frac{g_{s}^{2}}{(2 \pi)^{2}} \int_{0}^{1} d x \frac{1-x}{12} \frac{(1-x)^{3}(m+x M)}{\left[L_{s}^{2}\left(\Lambda_{s}^{2}\right)\right]^{3}} \equiv \int_{0}^{1} d x \kappa^{q(s)}(x) \\
\kappa^{q(a)} & =-\left.\frac{1}{q_{x}-i q_{y}} \int \frac{d \boldsymbol{p}_{T} d x}{16 \pi^{3}} \sum_{\lambda_{q}, \lambda_{a}}\left[\psi_{\lambda_{q} \lambda_{a}}^{+*}\left(x, \boldsymbol{p}_{T}^{\prime}\right) \psi_{\lambda_{q} \lambda_{a}}^{-}\left(x, \boldsymbol{p}_{T}\right)\right]\right|_{\boldsymbol{p}_{T}^{\prime}=\boldsymbol{p}_{T}} \\
& =-\frac{g_{a}^{2}}{(2 \pi)^{2}} \int_{0}^{1} d x \frac{x}{12} \frac{(1-x)^{3}(m+x M)}{\left[L_{a}^{2}\left(\Lambda_{a}^{2}\right)\right]^{3}} \equiv \int_{0}^{1} d x \kappa^{q(a)}(x)
\end{aligned}
$$

By comparison with the first $\boldsymbol{p}_{T}$-moment of $f_{1 T}^{\perp q(s)}$ and $f_{1 T}^{\perp q(a)}$ in Eqs. (98) and (99), respectively,

$$
\begin{aligned}
f_{1 T}^{\perp q(s)}(x) & =\int d \boldsymbol{p}_{T} f_{1 T}^{\perp q(s)}\left(x, \boldsymbol{p}_{T}^{2}\right) \\
& =-\frac{g_{s}^{2}}{(2 \pi)^{2}} \frac{M C_{F} \alpha_{s}(1-x)^{3}(m+x M)}{\left[2 L_{s}^{2}\left(\Lambda_{s}^{2}\right)\right]^{3}} \\
f_{1 T}^{\perp q(a)}(x) & =\int d \boldsymbol{p}_{T} f_{1 T}^{\perp q(a)}\left(x, \boldsymbol{p}_{T}^{2}\right) \\
& =\frac{g_{a}^{2}}{(2 \pi)^{2}} \frac{M C_{F} \alpha_{s} x(1-x)^{2}(m+x M)}{\left[2 L_{a}^{2}\left(\Lambda_{a}^{2}\right)\right]^{3}}
\end{aligned}
$$

we deduce the relation

$$
f_{1 T}^{\perp q}(x)=-\frac{3}{2} M C_{F} \alpha_{s} \frac{\kappa^{q}(x)}{1-x}
$$

valid for both types of diquarks, from which we have

$$
\int_{0}^{1} d x(1-x) f_{1 T}^{\perp q}(x)=-\frac{3}{2} M C_{F} \alpha_{s} \kappa^{q}
$$

that generalize the findings of Ref. [24, 26].

\section{NUMERICAL RESULTS AND COMPARISON WITH VARIOUS PARAMETRIZATIONS}

In this section, after fixing the parameters of the model by fitting some known distribution functions, we show the numerical results of our model for a few selected TMDs.

\section{A. Choice of model parameters}

In order to fix the parameters of the model, we try to reproduce the parametrizations of parton distribution functions extracted from experimental data. When doing this, however, we have to face the problem of choosing a scale $Q^{2}$ at which our model can be compared to the parametrization. In principle, this scale should be considered as a further parameter of the model. However, we checked that the lowest possible value of $Q^{2}$ is always preferred by the fit. This is not surprising, since probably the model is applicable to a very low scale, beyond the limit of applicability of the perturbative QCD evolution equations. Therefore, we have decided to compare it to a parametrization at the lowest possible value of $Q^{2}$.

For the unpolarized distribution functions $f_{1}^{u}$ and $f_{1}^{d}$, we have chosen the parametrization of the ZEUS collaboration 63] (ZEUS2002) at $Q_{0}^{2}=0.3 \mathrm{GeV}^{2}$. This set of PDFs gives also an estimate of the errors, which is important to perform a $\chi^{2}$ fit. Other parametrizations either do not reach such low $Q^{2}$ or provide no error estimate. 
For the helicity distributions $g_{1}^{u}$ and $g_{1}^{d}$, we chose the leading-order (LO) version from Ref. 64] (GRSV2000) at $Q^{2}=0.26 \mathrm{GeV}^{2}$. Since this parametrization comes with no error estimate, we assigned a fixed relative error of $10 \%$ and $25 \%$ to the up and down quark distributions, respectively, which is reasonably similar to the error estimates of other parametrizations at higher $Q^{2}$ (see, e.g., Ref. [65]).

Finally, in order to perform the fit we arbitrarily chose to select from each parametrization 25 equally spaced points in the range $x=0.1$ to 0.7 .

The free parameters of the model include the quark mass $m$, the nucleon-quark-diquark coupling $g_{X}$, the diquark mass $M_{X}$, and the cutoff $\Lambda_{X}$ in the nucleon-quark-diquark form factor, for $X=s, a$ scalar and axial-vector diquarks. It turns out that in order to achieve a good fit we need also to make a distinction between the two isospin states of the vector diquark. Hence, we will use $g_{a} M_{a}$, and $\Lambda_{a}$, for the coupling, mass and cutoff of the vector isoscalar diquark with $I_{3}=0$ (corresponding to the $u d$ system), and $g_{a}^{\prime}, M_{a}^{\prime}$, and $\Lambda_{a}^{\prime}$, for the normalization, mass and cutoff of the vector isovector diquark with $I_{3}=1$ (corresponding to the $u u$ system).

In order to reduce the number of free parameters, we decided to fix the value of the constituent quark mass to $m=0.3 \mathrm{GeV}$. We checked that the results are not very sensitive to the value of this parameter.

To perform the fit, we need to discuss the relation between the functions $f_{1}^{q(s)}, f_{1}^{q(a)}$ and $f_{1}^{q\left(a^{\prime}\right)}$, computed in the model, and the functions $f_{1}^{u}$ and $f_{1}^{d}$ of the global fits. For ease of interpretation, it is better to use normalized versions of the $f_{1}^{q(X)}$. Therefore, we write $f_{1 \text { norm }}^{q(X)}=\left(N_{X}^{2} / g_{X}^{2}\right) f_{1}^{q(X)}$ where $N_{X}$ are normalization constants determined by imposing

$$
\pi \int_{0}^{1} d x \int_{0}^{\infty} d \boldsymbol{p}_{T}^{2} f_{1 \text { norm }}^{q(X)}\left(x, \boldsymbol{p}_{T}^{2}\right)=1 .
$$

Quite generally, the relation between quark flavors and diquark types can be written as

$$
\begin{aligned}
& f_{1}^{u}=c_{s}^{2} f_{1 \text { norm }}^{u(s)}+c_{a}^{2} f_{1 \text { norm }}^{u(a)} \\
& f_{1}^{d}=c_{a}^{\prime 2} f_{1 \text { norm }}^{d\left(a^{\prime}\right)} .
\end{aligned}
$$

We will refer to the coefficients $c_{X}$ as "couplings", although they differ from the original couplings $g_{X}$ by the normalization constants $N_{X}$. They are free parameters of the model.

In past versions of the spectator diquark model [42], the quarks were assumed to occupy the lowest-energy available orbital with positive parity $\left(J^{P}=\frac{1}{2}^{+}\right)$; in this case, the proton wave function assumes an $\mathrm{SU}(4)=\mathrm{SU}(2) \otimes \mathrm{SU}(2)$ spinisospin symmetry, leading to probabilistic weights 3:1:2 among the scalar isoscalar (quark $u$ with diquark $s$ ), vector isoscalar (quark $u$ with diquark $a$ ), and vector isovector (quark $d$ with diquark $a^{\prime}$ ) configurations. Moreover, the overall size of the couplings was adjusted to give a total number of three quarks. These choices led to the relations [42]

$$
\begin{aligned}
f_{1}^{u} & =\frac{3}{2} f_{1 \text { norm }}^{u(s)}+\frac{1}{2} f_{1 \text { norm }}^{u(a)} \\
f_{1}^{d} & =f_{1 \text { norm }}^{d\left(a^{\prime}\right)} .
\end{aligned}
$$

There are two reasons to criticize this choice. First of all, in the present work the quark-diquark system can have a nonvanishing relative orbital angular momentum, as shown in the previous section. Thus, the proton wave function no longer displays an SU(4) symmetry. Secondly, strictly speaking the SU(4) decomposition gives coefficients that are three times smaller then the ones in the above relation. This is because the total number of quarks "seen" in the spectator model is only one, since the other two are always hidden inside the diquark. This is actually a fundamental limitation of the spectator model, it is independent of the SU(4) choice, and in our opinion it has not been sufficiently stressed in the literature. The only possible way out is to consider the diquark not as an elementary particle, but as formed by two quarks that can be also probed by the photon (see, e.g., Ref. [66]).

A different way to see this problem is by considering the (longitudinal) momentum sum rule. Since also the diquarks can carry momentum, they should be included in the corresponding sum rule. ${ }^{3}$ Using the handbag diagram of Fig. 2, we calculated the corresponding diquark distribution function $f_{1}^{X(q)}$ for the active diquark in the state $X$ and the spectator quark with flavor $q$, again using the first choice in Eq. (10) (independently of the choice of form factor). We found the remarkable property

$$
f_{1}^{X(q)}(x)=f_{1}^{q(X)}(1-x)
$$

\footnotetext{
3 A similar approach has been used in Ref. [25] to verify in the spectator model the validity of the so-called Burkardt sum rule [67], which is related to transverse-momentum conservation.
} 
By splitting the total proton momentum sum rule into the contributions of quarks, $P_{q}$, and of diquarks, $P_{X}$, using the symmetry property (126) we get

$$
\begin{aligned}
P_{q}+P_{X}= & \int_{0}^{1} d x x\left[c_{s}^{2} f_{1 \text { norm }}^{u(s)}(x)+c_{a}^{2} f_{1 \text { norm }}^{u(a)}(x)+c_{a}^{\prime 2} f_{1 \text { norm }}^{d\left(a^{\prime}\right)}(x)\right] \\
& +\int_{0}^{1} d x x\left[c_{s}^{2} f_{1 \text { norm }}^{s(u)}(x)+c_{a}^{2} f_{1 \text { norm }}^{a(u)}(x)+c_{a}^{\prime 2} f_{1 \text { norm }}^{a^{\prime}(d)}(x)\right] \\
= & \int_{0}^{1} d x\left[c_{s}^{2} f_{1 \text { norm }}^{u(s)}(x)+c_{a}^{2} f_{1 \text { norm }}^{u(a)}(x)+c_{a}^{\prime 2} f_{1 \text { norm }}^{d\left(a^{\prime}\right)}(x)\right] \\
= & c_{s}^{2}+c_{a}^{2}+c_{a}^{\prime 2} .
\end{aligned}
$$

It is therefore impossible in our spectator model to fulfill at the same time the momentum sum rule and the quark number sum rule.

Although from the fundamental point of view it is more important to satisfy the momentum sum rule, from the phenomenological point of view it is impossible to reproduce the parametrizations in a satisfactory way. We decided therefore to avoid imposing the momentum sum rule and let the fit choose the values of the parameters $c_{X}$.
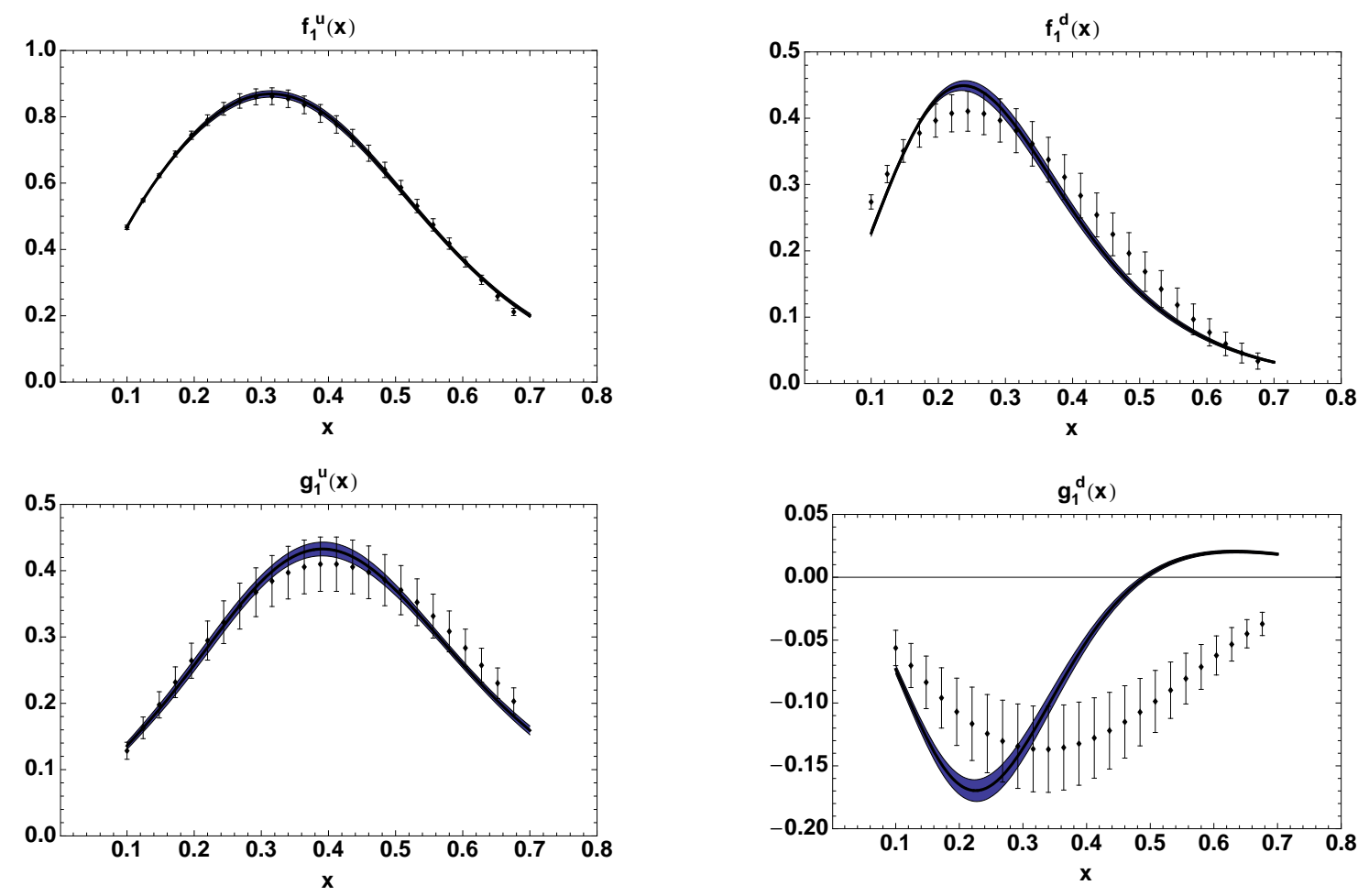

FIG. 4: The distribution functions $f_{1}(x)$ (above) and $g_{1}(x)$ (below) for the up quark (left panel) and the down quark (right panel). Data are a selection of 25 equidistant points in $0.1 \leq x \leq 0.7$ from the parametrizations of Ref. 663] (ZEUS2002) and Ref. 64] (GRSV2000) at LO, respectively (we assigned a constant relative error of $10 \%$ to $g_{1}^{u}$ and $25 \%$ to $g_{1}^{d}$ based on comparisons with similar fits [65] $)$. The curves represent the best fit $\left(\chi^{2} /\right.$ d.o.f. $\left.=3.88\right)$ obtained with our spectator model. The statistical uncertainty bands correspond to $\Delta \chi^{2}=1$.

In summary, we have 9 free parameters for the model. We fix them by fitting at the same time $f_{1}^{u}, f_{1}^{d}$ at $Q^{2}=0.3$ $\mathrm{GeV}^{2}$ from Ref. [63], and $g_{1}^{u}, g_{1}^{d}$ at $Q^{2}=0.26 \mathrm{GeV}^{2}$ from Ref. 64] at LO. The fit was performed using the MINUIT program. A $\chi^{2} /$ d.o.f. $=3.88$ was reached. The results are shown in Fig. 4. In spite of the very high $\chi^{2}$, the agreement is acceptable, except perhaps for the down quark helicity distribution. The error band is deduced from the covariance matrix given by MINUIT and represents the standard 1- $\sigma$ statistical uncertainty $\left(\Delta \chi^{2}=1\right)$. The corresponding values for the various model parameters are listed in Tab. I] 


\begin{tabular}{|c||c|c|c|}
\hline Diquark & $M_{X}(\mathrm{GeV})$ & $\Lambda_{X}(\mathrm{GeV})$ & $c_{X}$ \\
\hline Scalar $s(u u)$ & $0.822 \pm 0.053$ & $0.609 \pm 0.038$ & $0.847 \pm 0.111$ \\
\hline Axial-vector $a(u d)$ & $1.492 \pm 0.173$ & $0.716 \pm 0.074$ & $1.061 \pm 0.085$ \\
\hline Axial-vector $a^{\prime}(u u)$ & $0.890 \pm 0.008$ & $0.376 \pm 0.005$ & $0.880 \pm 0.008$ \\
\hline
\end{tabular}

TABLE I: Results for the model parameters with dipolar nucleon-quark-diquark form factor and light-cone transverse polarizations of the vector diquark: the diquark masses $M_{X}$, the cutoffs $\Lambda_{X}$ in the form factors, and the $c_{X}$ couplings for $X=s, a, a^{\prime}$ scalar isoscalar, vector isoscalar, and vector isovector diquarks. The fit was performed using the MINUIT program on the parametrization of $f_{1}(x)$ from Ref. 63] (ZEUS2002), and of $g_{1}(x)$ from Ref. 64] (GRSV2000) at LO, reaching a $\chi^{2} /$ d.o.f. $=$ 3.88 .
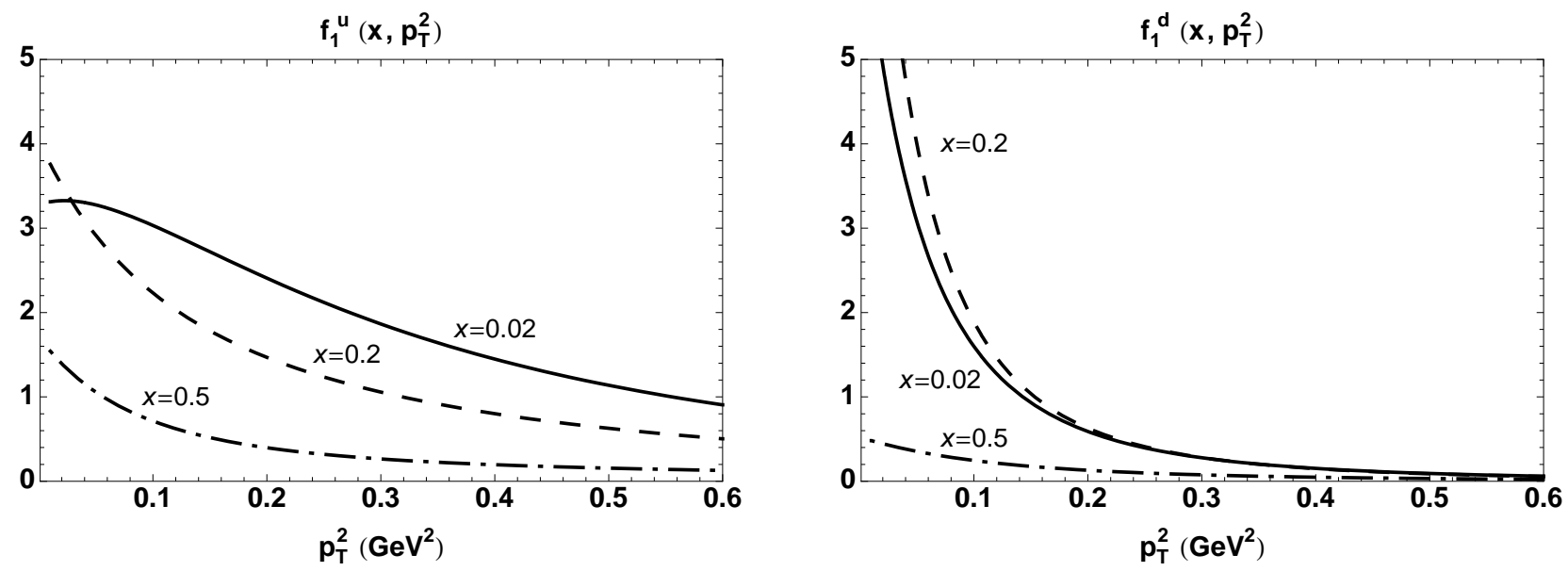

FIG. 5: The $\boldsymbol{p}_{T}^{2}$ dependence of the unpolarized distribution $f_{1}\left(x, \boldsymbol{p}_{T}^{2}\right)$ for up (left panel) and down quark (right panel). Different lines correspond to different values of $x$. The downturn of the function $f_{1}^{u}$ at relatively small $x$ is due to wavefunctions with nonzero orbital angular momentum.

\section{B. Unpolarized parton densities}

With the above model parameters, the proton momentum fraction $P_{q}$ carried by valence quarks, is

$$
\begin{aligned}
P_{q} & =\int_{0}^{1} d x x\left[c_{s}^{2} f_{1 \text { norm }}^{u(s)}(x)+c_{a}^{2} f_{1 \text { norm }}^{u(a)}(x)+c_{a}^{\prime 2} f_{1 \text { norm }}^{d\left(a^{\prime}\right)}(x)\right] \\
& =\int_{0}^{1} d x x\left[f_{1}^{u}(x)+f_{1}^{d}(x)\right] \approx 0.584 \pm 0.010,
\end{aligned}
$$

which is consistent with the ZEUS result of 0.5563 .

While for $f_{1}^{d}$ only the vector-isovector diquark plays a role, for $f_{1}^{u}$ it turns out that the contributions from the scalar and vector diquark have about the same size. The vector diquark is always dominant at high $x$. However, we know that the model is not reliable in the limit $x \rightarrow 1$. In fact, the behavior at high $x$ does not follow the predictions of Ref. [68], since our model does not correctly take into account the dominant dynamics in that region.

We consider now the $\boldsymbol{p}_{T}^{2}$ dependence of the unpolarized distribution function obtained in our model. In Fig. 5 we show the behavior of the up and down components as functions of $\boldsymbol{p}_{T}^{2}$ for a few values of the variable $x$.

First of all, we observe that $f_{1}^{u}$ displays a nonmonotonic behavior at $x \leq 0.02$. This is due to the contribution from LCWFs with nonzero orbital angular momentum. Although the details of where and how this feature occurs is model-dependent, it is generally true that the contribution of LCWFs with one unit of orbital angular momentum falls linearly with $\boldsymbol{p}_{T}^{2}$ for $\boldsymbol{p}_{T}^{2} \rightarrow 0$. This behavior is sharply different from the contribution of LCWFs with no orbital angular momentum. This simple example shows how the study of the $\boldsymbol{p}_{T}^{2}$ dependence of unpolarized TMDs can therefore already expose some effects due to orbital angular momentum.

Finally, we observe that in our model the average quark transverse momentum decreases as $x$ increases, and that down quarks on average carry less transverse momentum than up quarks. Although this is just a model result, a general message can be derived: the widely used assumption of a flavor-independent quark transverse momentum distribution is already falsified in a relatively simple model (see also Ref. [69]). 


\section{Longitudinally polarized parton densities}

The model parameters of Tab. I produce the axial charge

$$
g_{A}=\int_{0}^{1} d x\left[g_{1}^{u}(x)-g_{1}^{d}(x)\right]=0.966 \pm 0.038
$$

in excellent agreement with the value $0.969 \pm 0.096$ deduced from the GRSV parametrization [64].

It is, however, evident from Fig. 4 that our description of the down quark helicity distribution is in bad disagreement with the GRSV parametrization at large $x$. Nevertheless, we point out that there is a qualitative agreement with the parametrization of the so-called BBS model of Ref. [70] and the analogous parametrization of Ref. [68]. In particular, our model shows the same feature highlighted in this latter reference, namely that the contribution of the LCWFs with nonvanishing orbital angular momentum is dominant at high $x$. This is true in all distribution functions, but becomes particularly evident for the down helicity distribution, since the contribution from the LCWFs $\psi_{++}^{+}$and $\psi_{+-}^{+}$ (carrying nonzero orbital angular momentum) are positive and make the distribution positive at $x>0.5$.
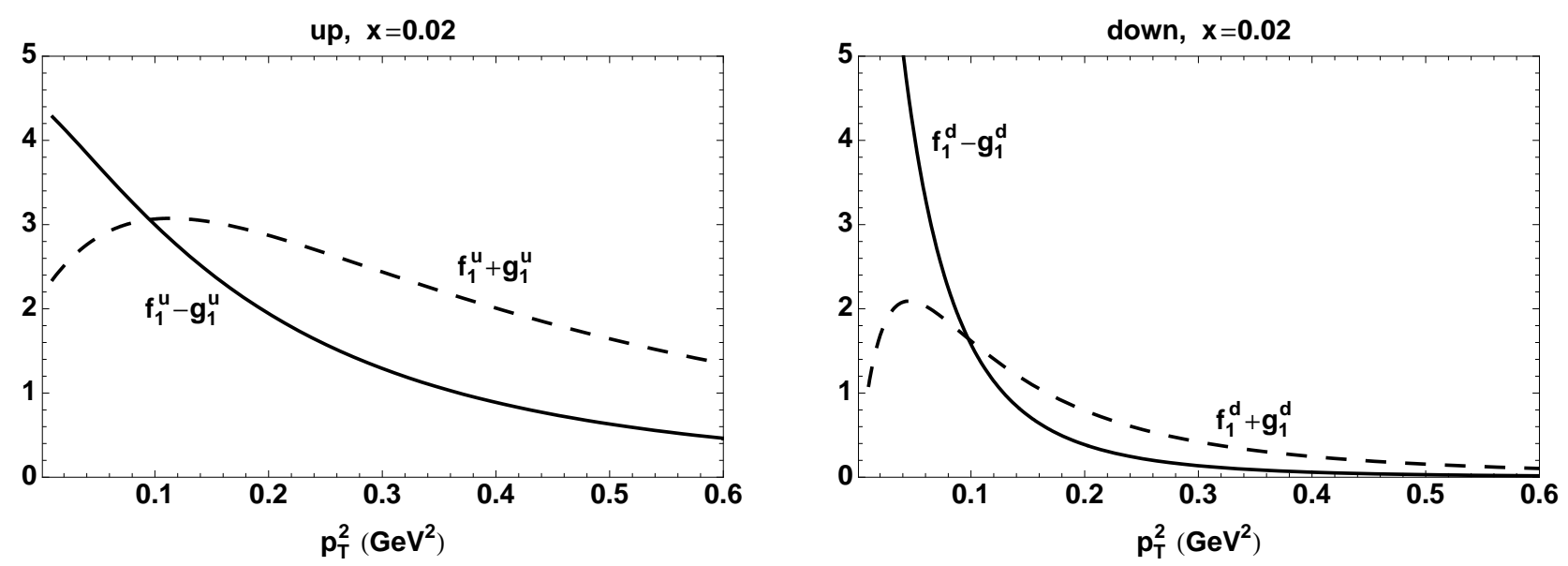

FIG. 6: The $\boldsymbol{p}_{T}^{2}$ dependence of the distributions $f_{1}\left(x, \boldsymbol{p}_{T}^{2}\right)-g_{1}\left(x, \boldsymbol{p}_{T}^{2}\right)$ (solid line) and $f_{1}\left(x, \boldsymbol{p}_{T}^{2}\right)+g_{1}\left(x, \boldsymbol{p}_{T}^{2}\right)($ dashed line) for up (left panel) and down quark (right panel), at $x=0.02$. The difference in their behavior is due to the different role played in the two combinations by wavefunctions with nonzero orbital angular momentum.

The effect of orbital angular momentum becomes even more evident when considering the $\boldsymbol{p}_{T}^{2}$ behavior of the helicity distribution function. As an illustration, we show in Fig. 6 the behavior of the combinations $f_{1}\left(x, \boldsymbol{p}_{T}^{2}\right)-g_{1}\left(x, \boldsymbol{p}_{T}^{2}\right)$ and $f_{1}\left(x, \boldsymbol{p}_{T}^{2}\right)+g_{1}\left(x, \boldsymbol{p}_{T}^{2}\right)$. In the case of the scalar diquark, LCWFs with one unit of orbital angular momentum are filtered by the first combination. In the case of the vector diquark, the situation is opposite. The down quark distribution is entirely given by the vector diquark, therefore the $f_{1}\left(x, \boldsymbol{p}_{T}^{2}\right)+g_{1}\left(x, \boldsymbol{p}_{T}^{2}\right)$ sum clearly turns down to zero for $\boldsymbol{p}_{T}^{2} \rightarrow 0$. For the up quark, the situation is less clear due to the simultaneous presence of scalar and vector diquark contributions. However, at $x=0.02$ the vector diquark is responsible for the nontrivial shape of $f_{1}\left(x, \boldsymbol{p}_{T}^{2}\right)+g_{1}\left(x, \boldsymbol{p}_{T}^{2}\right)$.

It is interesting also to investigate the $\boldsymbol{p}_{T}^{2}$ behavior of $g_{1}^{u}$ alone, shown in Fig. 7 . There is a dramatic change of behavior for different values of $x$, due to the difference between the scalar and vector diquark components of the function. If the spectator is a scalar diquark, for $\boldsymbol{p}_{T}=0$, where the LCWFs with orbital angular momentum vanish, the spin of the up quark has to be parallel to that of the proton, thus $g_{1}^{u(s)}(x, 0) \geq 0$. At high transverse momentum, where LCWFs with $L_{z}=1$ dominate, the spin of the up quark has to be antiparallel to that of the proton, thus $g_{1}^{u(s)}(x, \infty) \leq 0$. The situation is exactly reversed in the case of the vector diquark. As is already visible in Eqs. (58) and (59), at high transverse momentum the vector diquark always dominates and gives a positive result. At low transverse momentum, the relative size of the functions $L_{X}^{2}\left(\Lambda_{X}^{2}\right)$ in the denominator determines which contribution is dominant. At higher $x$ the scalar diquark dominates and gives a positive $g_{1}^{u}(x, 0)$, while at lower $x$ the vector diquark dominates and gives a negative $g_{1}^{u}(x, 0)$.

Once again, apart from the details specific to our model, these examples show that the exploration of the $\boldsymbol{p}_{T}^{2}$ dependence of the unpolarized and helicity distribution functions can expose very interesting features of the inner structure of the nucleon, related in particular to orbital angular momentum. 


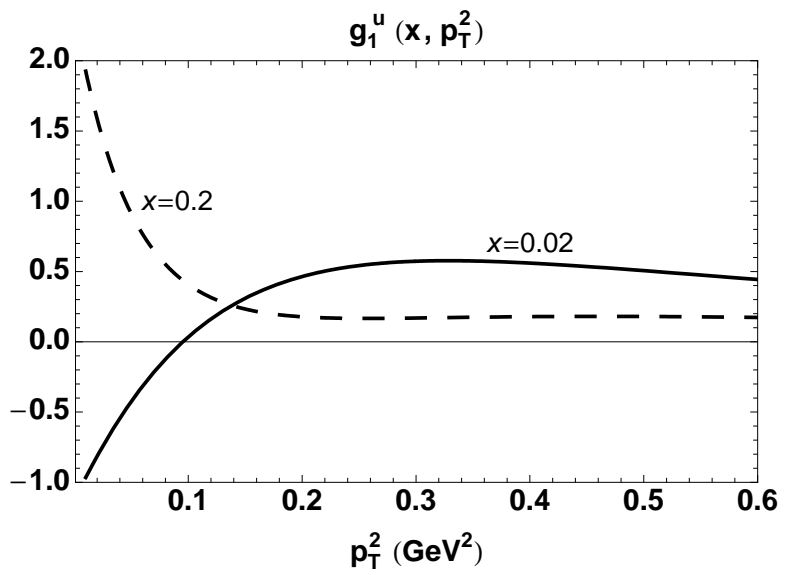

FIG. 7: The $\boldsymbol{p}_{T}^{2}$ dependence of the helicity distribution $g_{1}^{u}\left(x, \boldsymbol{p}_{T}^{2}\right)$. Different lines correspond to different values of $x$.
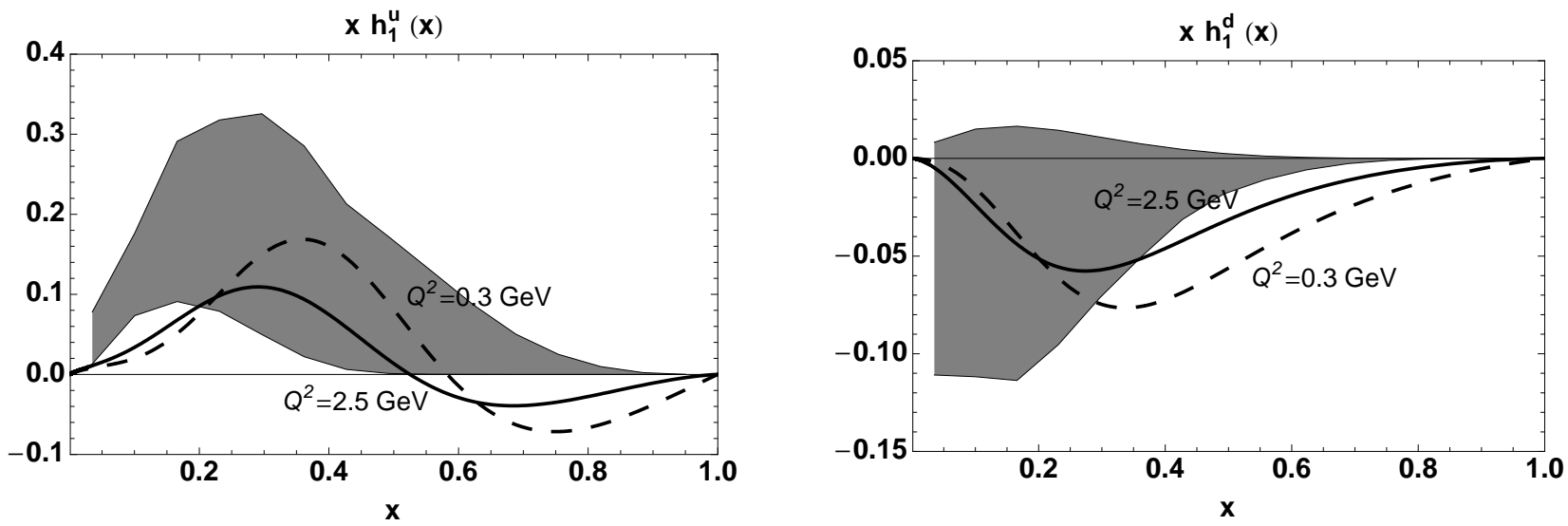

FIG. 8: The transversity distribution $x h_{1}(x)$ for up (left panel) and down quark (right panel). Dashed (solid) line for the model result before (after) the evolution at LO using the code of Ref. 71] up to the scale of the parametrization from Ref. 72], whose uncertainty band due to errors in the fit parameter is represented by the shaded area.

\section{Transversity}

In Fig. 8, the predictions of the spectator diquark model for the transversity distribution are compared with the only available parametrization of Ref. 72]. In the left panel, $x h_{1}^{u}(x)$ is shown, whereas $x h_{1}^{d}(x)$ is shown in the right panel. All the model results at the assumed original scale $Q_{0}^{2}=0.3 \mathrm{GeV}^{2}$ are represented by the dashed line. The solid line indicates the result after applying the DGLAP evolution at LO up to the scale $Q^{2}=2.5 \mathrm{GeV}^{2}$ using the code from Ref. [11]. The latter scale pertains the parametrization of Ref. [72], whose errors in the fit parameters produce the uncertainty band represented by the shaded area. The model is in reasonable agreement with the parametrization, with the maxima in the correct position and a somewhat too small result for the up quark at small $x$. It should also be kept in mind that the present data reach at most $x \approx 0.4$ [73, 74] and, moreover, the ansatz of Ref. 72] does not allow for a sign change. ${ }^{4}$

Interestingly, for the up quark the model predicts a change of sign at $x \sim 0.5$. To our knowledge, no other model of transversity displays this feature (see Ref. 76] and references therein; see also recent calculations in Refs. [66, 77, 78]).

\footnotetext{
${ }^{4}$ We point out that new fits of the transversity distribution functions have been presented at some conferences 75$]$ but not published yet.
} 
The reason for this sign change is that the contribution of the vector diquark is negative, as evident from Eq.(79). In our model, at moderate $x$ the scalar diquark contribution is dominant, whereas at sufficiently high $x$ the contribution of the vector diquark becomes in absolute size bigger, thus leading to the sign change. Other versions of the diquark model, even with vector diquarks, may not show this property. This is already evident from inspecting the results (listed in the appendices) for different choices of the diquark polarization sum. We don't think that our model calculation should be trusted more than others. Nevertheless, it might be interesting to contemplate the possibility of a sign change when choosing a form for the parametrization of the transversity function in "global fits."
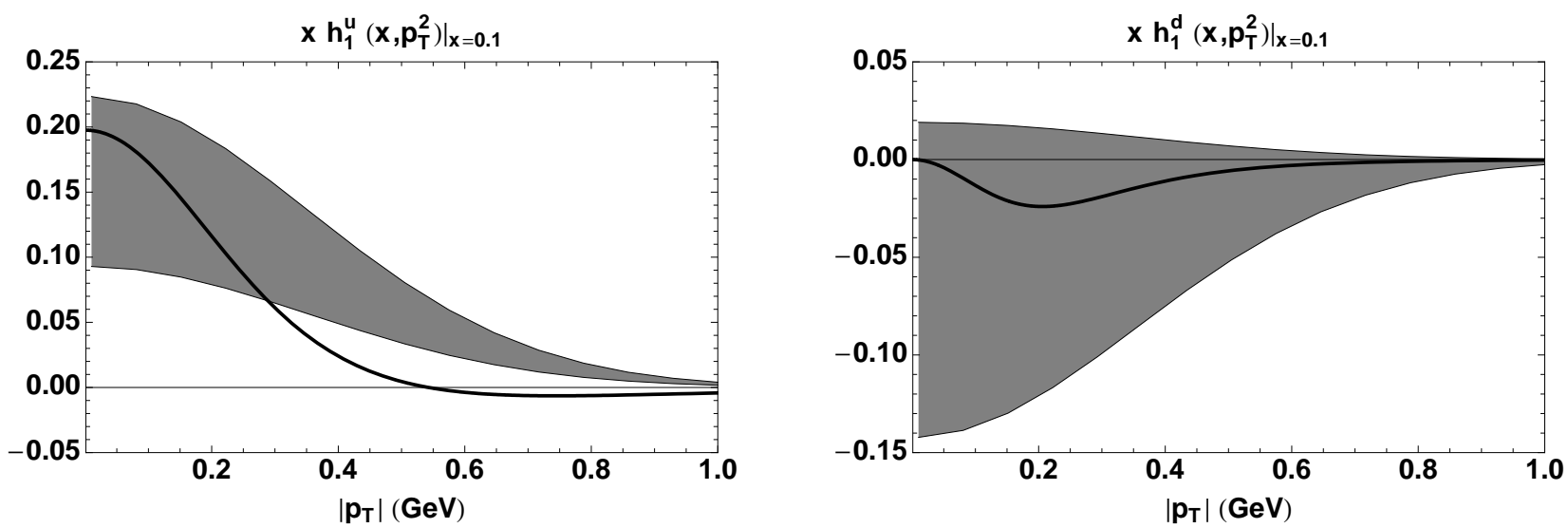

FIG. 9: Same as in the previous figure, but for the $\boldsymbol{p}_{T}$ dependence of transversity at $x=0.1$.

In Fig. 9] the same comparison is performed as in the previous figure, but for the $\boldsymbol{p}_{T}$ dependence of the transversity at $x=0.1$, as it is deduced from Eqs. (68]69). Again, there is a reasonable agreement between model predictions and parametrizations but for the trend of the result for the up quark at $\left|\boldsymbol{p}_{T}\right|>0.3 \mathrm{GeV} / c$. However, we stress that the comparison may be affected by the different scale of the model results $\left(Q^{2}=0.3 \mathrm{GeV}^{2}\right)$ and the one at which the parametrizations are extracted $\left(Q^{2}=2.5 \mathrm{GeV}^{2}\right)$. The proper evolution of the TMDs has not been considered yet. It is interesting to point out that in our model $h_{1}^{u}\left(x, \boldsymbol{p}_{T}^{2}\right)$ changes sign at $\boldsymbol{p}_{T} \sim 0.5 \mathrm{GeV}$. This is due to the fact that the vector diquark contribution is always negative and dominant at high $\boldsymbol{p}_{T}$. For the down quark, we note that $h_{1}^{d}(x, 0)=0$, because the vector-diquark contribution to $h_{1}$ is entirely given by LCWFs with nonvanishing orbital angular momentum.
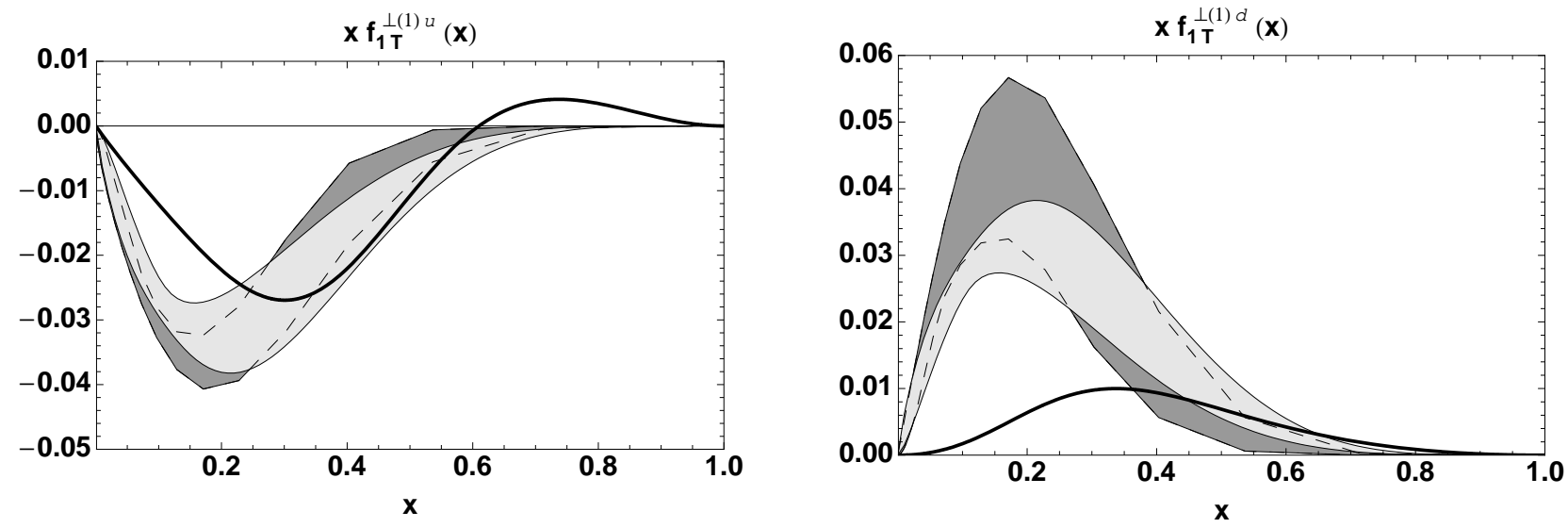

FIG. 10: The first $\boldsymbol{p}_{T}$-moment $x f_{1 T}^{\perp(1)}(x)$ of the Sivers function; left (right) panel for up (down) quark. Solid line for the results of the spectator diquark model. Darker shaded area for the uncertainty band due to the statistical error of the quark parametrizations from Ref. [79], lighter one from Ref. [80]. 


\section{E. Sivers function}

In Fig. 10, the $x f_{1 T}^{\perp(1)}(x)$ moment of the Sivers function, predicted using Eqs. (104) and (105), is given by the solid line and it is compared with two different parametrizations of the same observable. The darker shaded area represents the uncertainty due to the statistical errors in the parametrization of Ref. [79], while the lighter shaded area corresponds to the same for Ref. [80]. Left panel refers to the up quark, right panel to down quark. First of all, we observe the agreement between the signs of the various flavor components, which also agree with the findings from calculations on the lattice [81]. Also the maxima are reached at approximately the same $x \sim 0.3$ as the parametrizations. Instead, the "strength" of the asymmetry (related to the modulus of each moment) is too much weaker for the down quark, while it seems reasonable for the up one. Again, it must be stressed that no evolution was applied in the displayed model results.
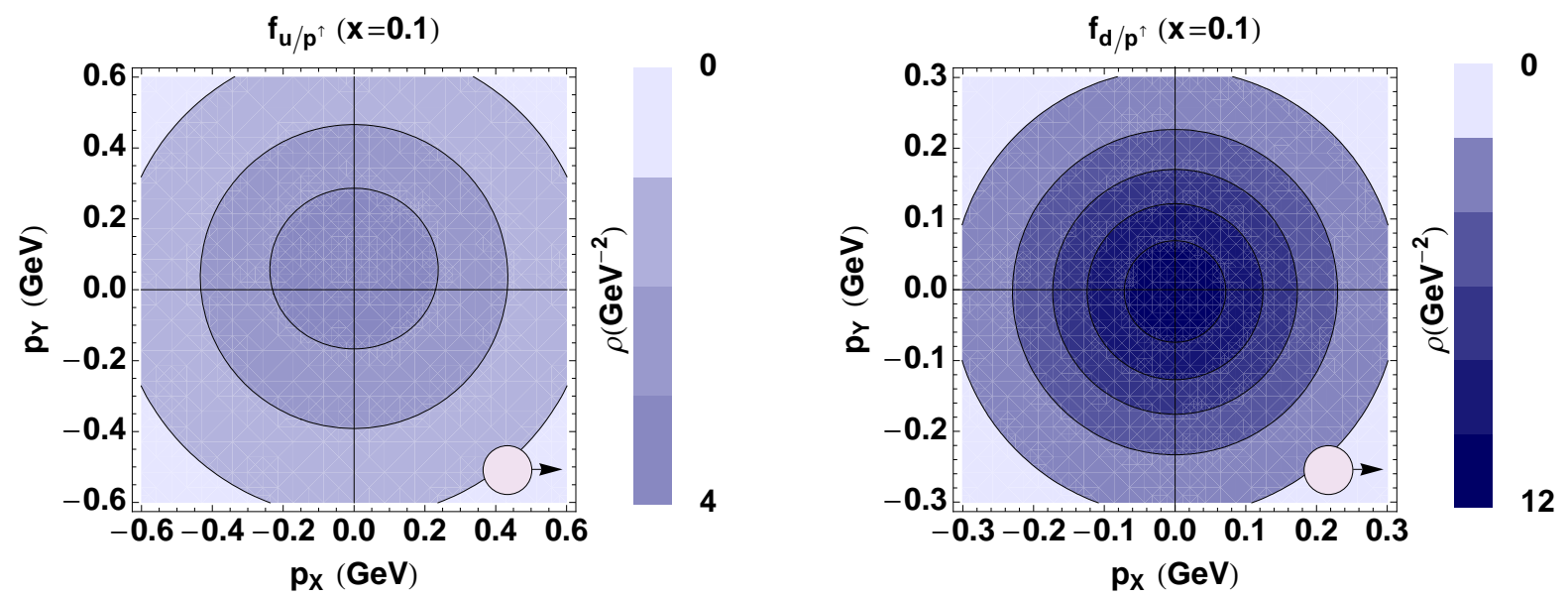

FIG. 11: The model result for the spin density of unpolarized quarks in transversely polarized protons (see text for the precise definition) in $\boldsymbol{p}_{T}$ space at $x=0.1$. Left panel for up quark, right panel for down quark. The circle with the arrow indicates the direction of the proton polarization.

According to the Trento conventions [62], we define the spin density of unpolarized quarks with flavor $q$ in transversely polarized protons as

$$
f_{q / p^{\uparrow}}\left(x, \boldsymbol{p}_{T}\right)=f_{1}^{q}\left(x, \boldsymbol{p}_{T}^{2}\right)-f_{1 T}^{\perp q}\left(x, \boldsymbol{p}_{T}^{2}\right) \frac{\left(\hat{\boldsymbol{P}} \times \boldsymbol{p}_{T}\right) \cdot \boldsymbol{S}}{M}
$$

In a SIDIS experiment, typically $\hat{\boldsymbol{P}}$ is antialigned to the $\hat{\boldsymbol{z}}$ axis that points in the direction of the momentum transfer $\boldsymbol{q}$. Hence, if the proton polarization is chosen along the $\hat{\boldsymbol{x}}$ axis, the spin density (130) shows an asymmetry in momentum space along the $p_{y}$ direction, whose size is driven by the Sivers function. In Fig. 11] we show $f_{q / p^{\uparrow}}\left(0.1, \boldsymbol{p}_{T}\right)$ for $q=u$ (left panel) and $q=d$ (right panel). Since the Sivers function for the up (down) quark is negative (positive), the density is deformed towards positive (negative) values of $p_{y}$. This feature is in agreement with the lattice results of Ref. [81] and with the signs of the anomalous magnetic moments $\kappa^{q}$ [61].

\section{F. Boer-Mulders function}

In Fig. 12, the $x h_{1}^{\perp(1)}(x)$ and $x h_{1}^{\perp{ }^{(1 / 2)}}(x)$ moments of the Boer-Mulders function, as deduced from Eqs. (106[107) and (110111), are displayed in the left and right panel, respectively. The solid lines correspond to the results for the up quark; dashed lines for the down quark. For the Boer-Mulders function, the only available parametrization appeared recently in [22], but the overall normalization depends on a parameter $\omega$ that cannot be fixed with available experimental information. Our result agrees in sign and shape with that extraction. The absolute values of our functions correspond to $\omega \approx 0.3$. We remark that there is full agreement between the sign of the $u$ and $d$ components and the aforementioned lattice calculations [81], as observed also in a different version of the spectator model [36] and 

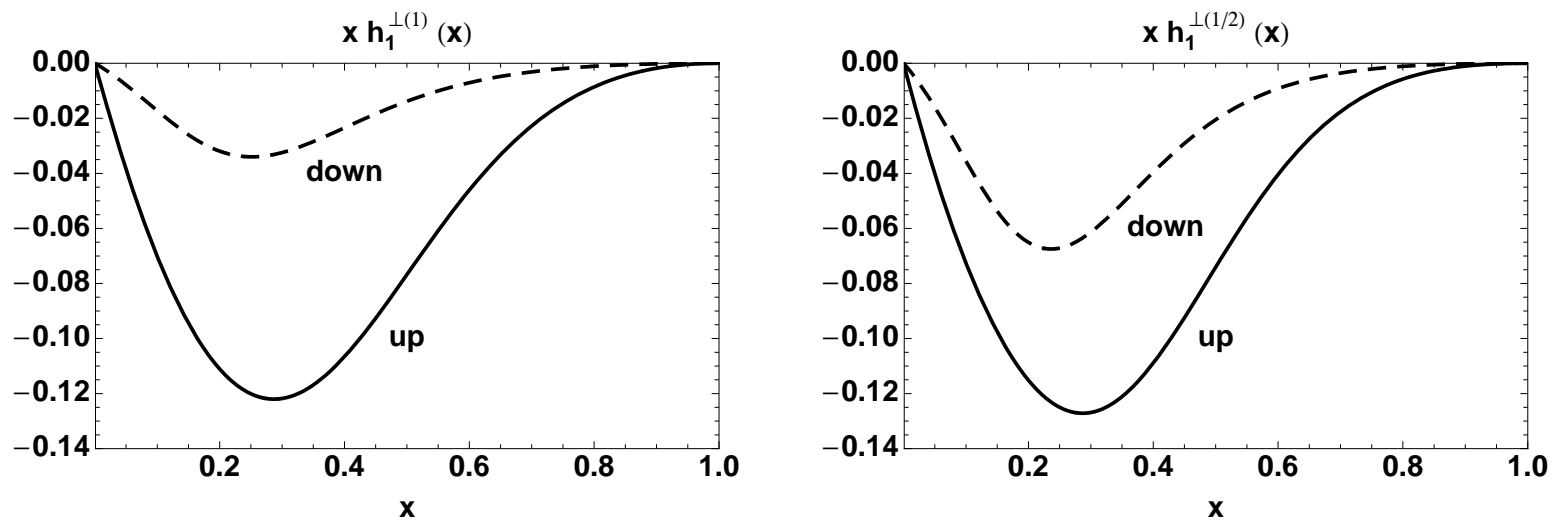

FIG. 12: The $x h_{1}^{\perp(1)}(x)$ (left) and $x h_{1}^{\perp(1 / 2)}(x)$ (right) moments of the Boer-Mulders function. Solid and dashed lines for up and down quarks, respectively.

in the bag model [47]. This agreement seems to be a general feature, as argued in Ref. [29]. ${ }^{5}$

In Fig. 13, we show, again at $x=0.1$, the spin density of transversely polarized quarks with flavor $q$ in unpolarized protons, related to the Boer-Mulders effect by [62]

$$
f_{q^{\uparrow} / p}\left(x, \boldsymbol{p}_{T}\right)=\frac{1}{2}\left[f_{1}^{q}\left(x, \boldsymbol{p}_{T}^{2}\right)-h_{1}^{\perp q}\left(x, \boldsymbol{p}_{T}^{2}\right) \frac{\left(\hat{\boldsymbol{P}} \times \boldsymbol{p}_{T}\right) \cdot \boldsymbol{S}_{q}}{M}\right],
$$

where now the quark polarization $\boldsymbol{S}_{q}$ points along $\hat{\boldsymbol{x}}$. Since the Boer-Mulders function is negative for both flavors (see Fig. [12), the related spin density is always deformed towards positive $p_{y}$, again in agreement with the lattice results [81].
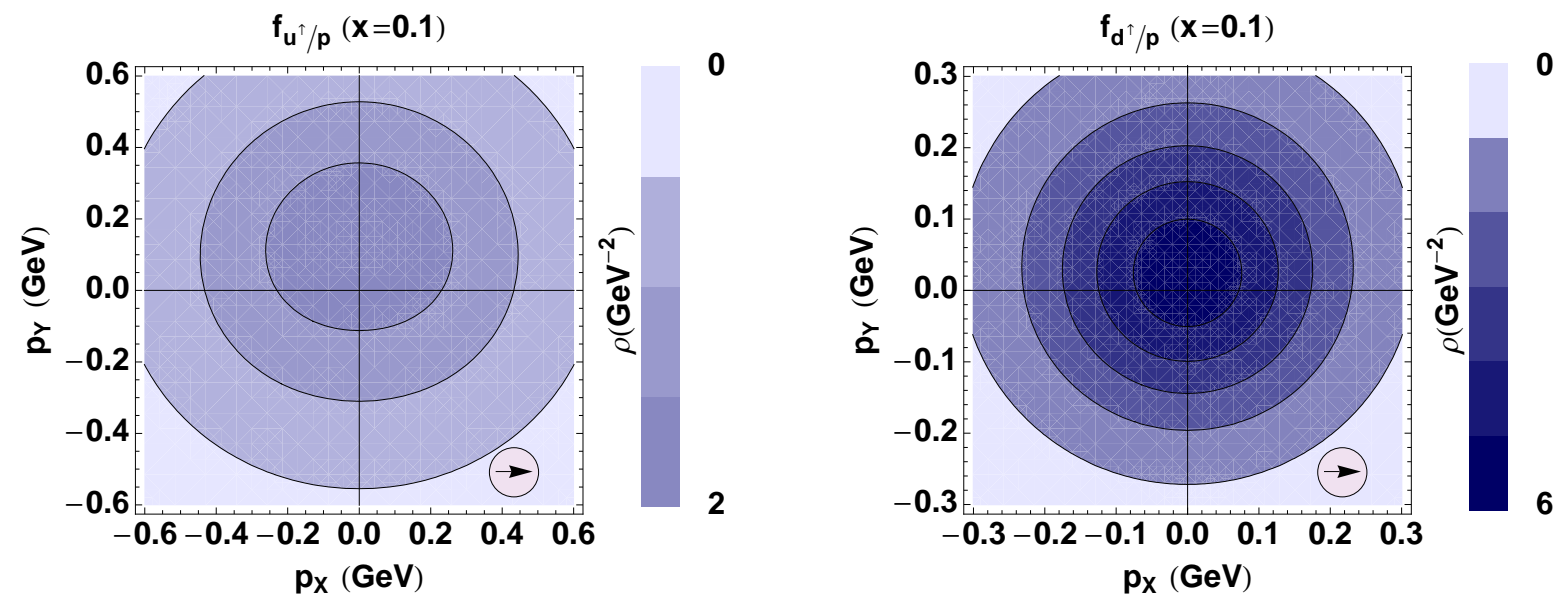

FIG. 13: The model result for the spin density of transversely polarized quarks in unpolarized protons (see text for the precise definition) in $\boldsymbol{p}_{T}$ space at $x=0.1$. Left panel for up quark, right panel for down quark. The arrow inside the circle indicates the direction of the quark polarization.

\footnotetext{
${ }^{5}$ A different result for the sign of the down quark Boer-Mulders function was obtained in Ref. [46], probably due to a mistake in that calculation (see App. B4).
} 


\section{CONCLUSIONS}

We have presented a systematic calculation of all leading-twist parton distributions in the nucleon in a diquark spectator model. We have generated the relative phase necessary to produce T-odd structures by approximating the gauge link operator with a one gluon-exchange interaction. All results have been presented in analytic form and interpreted in terms of overlaps of light-cone wavefunctions.

We tried to extend and improve the spectator model calculations presented in Refs. [36, 42, 46] by considering several choices of the axial-vector diquark polarization states and of the nucleon-quark-diquark form factor. We listed the analytic expressions for all possible choices in the appendices. We critically reconsidered some of the limits of the model and the choice of model parameters used in the past literature. In particular, we showed that the spectator diquark model is not able to reproduce both the quark number and momentum sum rule at the same time, because the diquark is considered as a charged parton, hence active in the sum rules. We argued that the proton wave function does not show the usual $\mathrm{SU}(4)=\mathrm{SU}(2) \otimes \mathrm{SU}(2)$ symmetry [42], since the quark-diquark system in its ground state can have a nonvanishing relative orbital angular momentum.

For numerical studies, we chose the version of the model that in our opinion is more sensible and practical, i.e., the one where only light-cone transverse polarizations of the diquark are present and a dipolar form factor is used. We identified nine free parameters of the model and we fixed them by reproducing the phenomenological parametrization of unpolarized [63] and longitudinally polarized [64] parton distributions at the lowest available scale, i.e. $Q^{2}=0.3$ and $0.26 \mathrm{GeV}^{2}$, respectively.

Whenever possible, results have been compared with available parametrizations. For the chiral-odd transversity distribution, there is only one available from Ref. [72], which was deduced from SSA data at $Q^{2}=2.5 \mathrm{GeV}^{2}$. The $\boldsymbol{p}_{T}$-integrated model result, once evolved to this scale using the code from Ref. 71] at LO, displays a satisfactory overall agreement. The $f_{1 T}^{\perp(1)}(x)$ moment of the chiral-even T-odd Sivers function $f_{1 T}^{\perp}$ was compared with the parametrizations of Refs. [79, 80]. There is agreement between the signs of the various flavor components and between the positions of the maxima in $x$, but the absolute value of the function is somewhat too small for the down quark. The comparison is affected by the difference of the scales, since evolution equations for the Sivers function

have not been used. We also plotted the $h_{1}^{\perp(1)}(x)$ and $h_{1}^{\perp(1 / 2)}(x)$ moments of the chiral-odd T-odd Boer-Mulders function $h_{1}^{\perp}$. We have also shown the quark spin densities defined in the Trento conventions 62], as produced in turn by the Sivers or the Boer-Mulders effects. For unpolarized quarks in transversely polarized protons, the spin density $f_{q / p^{\uparrow}}$ is linked to $f_{1 T}^{\perp}$, while for transversely polarized quarks in unpolarized protons the $f_{q^{\uparrow} / p}$ is linked to $h_{1}^{\perp}$. For transverse polarizations along the $\hat{\boldsymbol{x}}$ axis, the contour plot in the quark momentum space of such densities at $x=0.1$ displays a distortion in the $p_{y}$ direction, whose sign is consistent with the lattice findings for the corresponding spin densities in impact parameter space [81].

Using the model parton densities discussed above, various spin, beam, and azimuthal asymmetries in semi-inclusive hadronic reactions can be predicted, which are of interest for several experimental collaborations. Model calculations can be useful to interpret experimental measurements, helping us to explore spin-orbit parton correlations inside hadrons and shed light on the well-known puzzle of the proton spin sum rule.

\section{Acknowledgments}

F. C. and M. R. would like to thank B. Pasquini for useful discussions.

This work is part of the European Integrated Infrastructure Initiative in Hadronic Physics project under Contract No. RII3-CT-2004-506078.

Authored by Jefferson Science Associates, LLC under U.S. DOE Contract No. DE-AC05-06OR23177. The U.S. Government retains a non-exclusive, paid-up, irrevocable, world-wide license to publish or reproduce this manuscript for U.S. Government purposes.

\section{APPENDIX A: T-EVEN FUNCTIONS IN DIFFERENT VARIATIONS OF THE MODEL}

In this Appendix we list the leading-twist T-even parton densities obtained in the context of our spectator diquark model, for all the choices of axial-vector diquark polarization sum and nucleon-quark-diquark vertex. To avoid overloading the notation, we will use the same ones for the parameters involved $\left(g_{X}, M_{X}, \Lambda_{X}\right)$. However, it must be kept in mind that the numerical value of these parameters can be different in the various versions of the model. 


\section{Scalar diquark}

The results for the scalar diquark are

$$
\begin{aligned}
f_{1}^{q(s)}\left(x, \boldsymbol{p}_{T}^{2}\right) & =\frac{g_{s}^{2}}{(2 \pi)^{3}} \frac{\left[(m+x M)^{2}+\boldsymbol{p}_{T}^{2}\right](1-x)}{2\left[\boldsymbol{p}_{T}^{2}+L_{s}^{2}\left(m^{2}\right)\right]^{2}} \\
g_{1 L}^{q(s)}\left(x, \boldsymbol{p}_{T}^{2}\right) & =\frac{g_{s}^{2}}{(2 \pi)^{3}} \frac{\left[(m+x M)^{2}-\boldsymbol{p}_{T}^{2}\right](1-x)}{2\left[\boldsymbol{p}_{T}^{2}+L_{s}^{2}\left(m^{2}\right)\right]^{2}}, \\
g_{1 T}^{q(s)}\left(x, \boldsymbol{p}_{T}^{2}\right) & =\frac{g_{s}^{2}}{(2 \pi)^{3}} \frac{M(m+x M)(1-x)}{\left[\boldsymbol{p}_{T}^{2}+L_{s}^{2}\left(m^{2}\right)\right]^{2}}, \\
h_{1 L}^{\perp q(s)}\left(x, \boldsymbol{p}_{T}^{2}\right) & =-g_{1 T}^{q(s)}\left(x, \boldsymbol{p}_{T}^{2}\right), \\
h_{1 T}^{q(s)}\left(x, \boldsymbol{p}_{T}^{2}\right) & =f_{1}^{q(s)}\left(x, \boldsymbol{p}_{T}^{2}\right), \\
h_{1 T}^{\perp q(s)}\left(x, \boldsymbol{p}_{T}^{2}\right) & =-\frac{g_{s}^{2}}{(2 \pi)^{3}} \frac{M^{2}(1-x)}{\left[\boldsymbol{p}_{T}^{2}+L_{s}^{2}\left(m^{2}\right)\right]^{2}},
\end{aligned}
$$

where we recall that $M$ is the nucleon mass and $m$ is the mass of the parton. From the latter two densities, we construct the contribution of the scalar diquark to the transversity:

$$
\begin{aligned}
h_{1}^{q(s)}\left(x, \boldsymbol{p}_{T}^{2}\right) & =h_{1 T}^{q(s)}\left(x, \boldsymbol{p}_{T}^{2}\right)+\frac{\boldsymbol{p}_{T}^{2}}{2 M^{2}} h_{1 T}^{\perp q(s)}\left(x, \boldsymbol{p}_{T}^{2}\right) \\
& =\frac{g_{s}^{2}}{(2 \pi)^{3}} \frac{(m+x M)^{2}(1-x)}{2\left[\boldsymbol{p}_{T}^{2}+L_{s}^{2}\left(m^{2}\right)\right]^{2}}=\frac{1}{2}\left(f_{1}^{q(s)}\left(x, \boldsymbol{p}_{T}^{2}\right)+g_{1}^{q(s)}\left(x, \boldsymbol{p}_{T}^{2}\right)\right) .
\end{aligned}
$$

The above results are valid for a point-like nucleon-quark-diquark coupling. For the other form factors it is sufficient to apply the replacements

$$
\begin{array}{ll}
g_{s}^{2} \rightarrow g_{s}^{2}(1-x)^{2} \frac{\left[\boldsymbol{p}_{T}^{2}+L_{s}^{2}\left(m^{2}\right)\right]^{2}}{\left[\boldsymbol{p}_{T}^{2}+L_{s}^{2}\left(\Lambda_{s}^{2}\right)\right]^{4}} & \text { dipolar form factor }, \\
g_{s}^{2} \rightarrow g_{s}^{2} e^{-\left[\boldsymbol{p}_{T}^{2}+L_{X}^{2}\left(m^{2}\right)\right] /\left[(1-x) \Lambda_{X}^{2}\right]} & \text { exponential form factor. }
\end{array}
$$

The integrated results are obviously different for the three form-factor choices. In all cases the transversity function saturates the Soffer bound, i.e.,

$$
h_{1}^{q(s)}(x)=\frac{1}{2}\left(f_{1}^{q(s)}(x)+g_{1}^{q(s)}(x)\right)
$$

- Point-like coupling (to avoid divergences we assume that the $\boldsymbol{p}_{T}^{2}$ integration is extended up to a finite cutoff $\Lambda_{s}^{2}$ )

$$
\begin{aligned}
& f_{1}^{q(s)}(x)=\frac{g_{s}^{2}(1-x)}{(2 \pi)^{2}} \frac{(m+x M)^{2} \Lambda_{s}^{2}-L_{s}^{2}\left(m^{2}\right) \Lambda_{s}^{2}+L_{s}^{2}\left(m^{2}\right)\left[\Lambda_{s}^{2}+L_{s}^{2}\left(m^{2}\right)\right] \log \left(\frac{\Lambda_{s}^{2}}{L_{s}^{2}\left(m^{2}\right)}+1\right)}{4 L_{s}^{2}\left(m^{2}\right)\left[\Lambda_{s}^{2}+L_{s}^{2}\left(m^{2}\right)\right]} \\
& g_{1}^{q(s)}(x)=\frac{g_{s}^{2}(1-x)}{(2 \pi)^{2}} \frac{(m+x M)^{2} \Lambda_{s}^{2}+L_{s}^{2}\left(m^{2}\right) \Lambda_{s}^{2}-L_{s}^{2}\left(m^{2}\right)\left[\Lambda_{s}^{2}+L_{s}^{2}\left(m^{2}\right)\right] \log \left(\frac{\Lambda_{s}^{2}}{L_{s}^{2}\left(m^{2}\right)}+1\right)}{4 L_{s}^{2}\left(m^{2}\right)\left[\Lambda_{s}^{2}+L_{s}^{2}\left(m^{2}\right)\right]}
\end{aligned}
$$

- Dipolar form factor [same as Eqs. (74) and (76)]

$$
\begin{aligned}
& f_{1}^{q(s)}(x)=\frac{g_{s}^{2}}{(2 \pi)^{2}} \frac{\left[2(m+x M)^{2}+L_{s}^{2}\left(\Lambda_{s}^{2}\right)\right](1-x)^{3}}{24 L_{s}^{6}\left(\Lambda_{s}^{2}\right)} \\
& g_{1}^{q(s)}(x)=\frac{g_{s}^{2}}{(2 \pi)^{2}} \frac{\left[2(m+x M)^{2}-L_{s}^{2}\left(\Lambda_{s}^{2}\right)\right](1-x)^{3}}{24 L_{s}^{6}\left(\Lambda_{s}^{2}\right)}
\end{aligned}
$$


- Exponential form factor

$$
\begin{gathered}
f_{1}^{q(s)}(x)=\frac{g_{s}^{2}}{(2 \pi)^{2}} \frac{1}{4}\left\{e^{-2 L_{s}^{2}\left(m^{2}\right) /\left[(1-x) \Lambda_{s}^{2}\right]} \frac{1-x}{L_{s}^{2}\left(m^{2}\right)}\left[(m+x M)^{2}-L_{s}^{2}\left(m^{2}\right)\right]\right. \\
\left.-\Gamma\left(0, \frac{2 L_{s}^{2}\left(m^{2}\right)}{(1-x) \Lambda_{s}^{2}}\right) \frac{2\left[(m+x M)^{2}-L_{s}^{2}\left(m^{2}\right)\right]-(1-x) \Lambda_{s}^{2}}{\Lambda_{s}^{2}}\right\}, \\
g_{1}^{q(s)}(x)=\frac{g_{s}^{2}}{(2 \pi)^{2}} \frac{1}{4}\left\{e^{-2 L_{s}^{2}\left(m^{2}\right) /\left[(1-x) \Lambda_{s}^{2}\right]} \frac{1-x}{L_{s}^{2}\left(m^{2}\right)}\left[(m+x M)^{2}+L_{s}^{2}\left(m^{2}\right)\right]\right. \\
\left.-\Gamma\left(0, \frac{2 L_{s}^{2}\left(m^{2}\right)}{(1-x) \Lambda_{s}^{2}}\right) \frac{2\left[(m+x M)^{2}+L_{s}^{2}\left(m^{2}\right)\right]+\Lambda_{s}^{2}(1-x)}{\Lambda_{s}^{2}}\right\},
\end{gathered}
$$

where $\Gamma$ is the incomplete $\Gamma$ function, defined as

$$
\Gamma(a, z)=\int_{z}^{\infty} t^{a-1} e^{-t} d t .
$$

\section{Axial-vector diquark with light-cone transverse polarization only}

The unintegrated parton densities are

$$
\begin{aligned}
f_{1}^{q(a)}\left(x, \boldsymbol{p}_{T}^{2}\right) & =\frac{g_{a}^{2}}{(2 \pi)^{3}} \frac{\boldsymbol{p}_{T}^{2}\left(1+x^{2}\right)+(m+x M)^{2}(1-x)^{2}}{2\left[\boldsymbol{p}_{T}^{2}+L_{a}^{2}\left(m^{2}\right)\right]^{2}(1-x)} \\
g_{1 L}^{q(a)}\left(x, \boldsymbol{p}_{T}^{2}\right) & =\frac{g_{a}^{2}}{(2 \pi)^{3}} \frac{\boldsymbol{p}_{T}^{2}\left(1+x^{2}\right)-(m+x M)^{2}(1-x)^{2}}{2\left[\boldsymbol{p}_{T}^{2}+L_{a}^{2}\left(m^{2}\right)\right]^{2}(1-x)}, \\
g_{1 T}^{q(a)}\left(x, \boldsymbol{p}_{T}^{2}\right) & =\frac{g_{a}^{2}}{(2 \pi)^{3}} \frac{M x(m+x M)}{\left[\boldsymbol{p}_{T}^{2}+L_{a}^{2}\left(m^{2}\right)\right]^{2}}, \\
h_{1 L}^{\perp q(a)}\left(x, \boldsymbol{p}_{T}^{2}\right) & =g_{1 T}^{q(a)}\left(x, \boldsymbol{p}_{T}^{2}\right) / x, \\
h_{1 T}^{q(a)}\left(x, \boldsymbol{p}_{T}^{2}\right) & =-\frac{g_{a}^{2}}{(2 \pi)^{3}} \frac{x \boldsymbol{p}_{T}^{2}}{\left[\boldsymbol{p}_{T}^{2}+L_{a}^{2}\left(m^{2}\right)\right]^{2}(1-x)}, \\
h_{1 T}^{\perp q(a)}\left(x, \boldsymbol{p}_{T}^{2}\right) & =0, \\
h_{1}^{q(a)}\left(x, \boldsymbol{p}_{T}^{2}\right) & =h_{1 T}^{q(a)}\left(x, \boldsymbol{p}_{T}^{2}\right) .
\end{aligned}
$$

The above results are valid for a point-like nucleon-quark-diquark coupling. For the other form factors it is sufficient to apply the replacements in Eqs. (A8) and (A9).

The integrated results are

- Point-like coupling (to avoid divergences we assume that the $\boldsymbol{p}_{T}^{2}$ integration is extended up to a finite cutoff $\Lambda_{a}^{2}$ )

$$
\begin{aligned}
f_{1}^{q(a)}(x)= & \frac{g_{a}^{2}}{(2 \pi)^{2}} \frac{1}{4 L_{a}^{2}\left(m^{2}\right)\left[\Lambda_{a}^{2}+L_{a}^{2}\left(m^{2}\right)\right](1-x)}\left[x \Lambda_{a}^{2}\left[\left(M^{2}-m^{2}\right)\left(1-x^{2}\right)+2 m M(1-x)^{2}-M_{a}^{2}\left(1+x^{2}\right)\right]\right. \\
& \left.+L_{a}^{2}\left(m^{2}\right)\left[\Lambda_{a}^{2}+L_{a}^{2}\left(m^{2}\right)\right]\left(1+x^{2}\right) \log \left(\frac{\Lambda_{a}^{2}}{L_{a}^{2}\left(m^{2}\right)}+1\right)\right], \\
g_{1}^{q(a)}(x)= & \frac{g_{a}^{2}}{(2 \pi)^{2}} \frac{1}{4 L_{a}^{2}\left(m^{2}\right)\left[\Lambda_{a}^{2}+L_{a}^{2}\left(m^{2}\right)\right](1-x)}\left\{L_{a}^{2}\left(m^{2}\right)\left[\Lambda_{a}^{2}+L_{a}^{2}\left(m^{2}\right)\right]\left(1+x^{2}\right) \log \left(\frac{\Lambda_{a}^{2}}{L_{a}^{2}\left(m^{2}\right)}+1\right)\right. \\
& \left.-\Lambda_{a}^{2}\left[(1-x) m M[x(1-x)(2 M-m)+2 m]+x(1-x) M^{3}\left(x-2 x^{2}-1\right)+x\left(1+x^{2}\right) M M_{a}^{2}\right]\right\},
\end{aligned}
$$




$$
h_{1}^{q(a)}(x)=-\frac{g_{a}^{2}}{(2 \pi)^{2}} \frac{x\left[\Lambda_{a}^{2}\left[(1-x)\left(x M^{2}-m^{2}\right)-x M_{a}^{2}\right]+L_{a}^{2}\left(m^{2}\right)\left[\Lambda_{a}^{2}+L_{a}^{2}\left(m^{2}\right)\right] \log \left(\frac{\Lambda_{a}^{2}}{L_{a}^{2}\left(m^{2}\right)}+1\right)\right]}{2 L_{a}^{2}\left(m^{2}\right)\left[\Lambda_{a}^{2}+L_{a}^{2}\left(m^{2}\right)\right](1-x)} .
$$

- Dipolar form factor [same as Eqs. (75177, 79)]

$$
\begin{aligned}
& f_{1}^{q(a)}(x)=\frac{g_{a}^{2}}{(2 \pi)^{2}} \frac{\left[2(m+x M)^{2}(1-x)^{2}+\left(1+x^{2}\right) L_{a}^{2}\left(\Lambda_{a}^{2}\right)\right](1-x)}{24 L_{a}^{6}\left(\Lambda_{a}^{2}\right)}, \\
& g_{1}^{q(a)}(x)=-\frac{g_{a}^{2}}{(2 \pi)^{2}} \frac{\left[2(m+x M)^{2}(1-x)^{2}-\left(1+x^{2}\right) L_{a}^{2}\left(\Lambda_{a}^{2}\right)\right](1-x)}{24 L_{a}^{6}\left(\Lambda_{a}^{2}\right)} \\
& h_{1}^{q(a)}(x)=-\frac{g_{a}^{2}}{(2 \pi)^{2}} \frac{x(1-x)}{12 L_{a}^{4}\left(\Lambda_{a}^{2}\right)} .
\end{aligned}
$$

- Exponential form factor

$$
\begin{aligned}
& f_{1}^{q(a)}(x)=\frac{g_{a}^{2}}{(2 \pi)^{2}} \frac{1}{4 \Lambda_{a}^{2} L_{a}^{2}\left(m^{2}\right)(1-x)^{2}}\left\{\Lambda_{a}^{2} L_{a}^{2}\left(m^{2}\right)(1-x)\left(1+x^{2}\right) \Gamma\left(0,2 \frac{L_{a}^{2}\left(m^{2}\right)}{(1-x) \Lambda_{a}^{2}}\right)\right. \\
& +\left[(m+x M)^{2}(1-x)^{2}-L_{a}^{2}\left(m^{2}\right)\left(1+x^{2}\right)\right] \\
& \left.\times\left[(1-x) \Lambda_{a}^{2} e^{-2 L_{a}^{2}\left(m^{2}\right) /\left[(1-x) \Lambda_{a}^{2}\right]}-2 L_{a}^{2}\left(m^{2}\right) \Gamma\left(0,2 \frac{L_{a}^{2}\left(m^{2}\right)}{(1-x) \Lambda_{a}^{2}}\right)\right]\right\}, \\
& g_{1}^{q(a)}(x)=\frac{g_{a}^{2}}{(2 \pi)^{2}} \frac{1}{4 \Lambda_{a}^{2} L_{a}^{2}\left(m^{2}\right)(1-x)^{2}}\left\{\Lambda_{a}^{2} L_{a}^{2}\left(m^{2}\right)(1-x)\left(1+x^{2}\right) \Gamma\left(0,2 \frac{L_{a}^{2}\left(m^{2}\right)}{(1-x) \Lambda_{a}^{2}}\right)\right. \\
& +\left[(m+x M)^{2}(1-x)^{2}+L_{a}^{2}\left(m^{2}\right)\left(1+x^{2}\right)\right] \\
& \left.\times\left[2 L_{a}^{2}\left(m^{2}\right) \Gamma\left(0,2 \frac{L_{a}^{2}\left(m^{2}\right)}{(1-x) \Lambda_{a}^{2}}\right)-(1-x) \Lambda_{a}^{2} e^{-2 L_{a}^{2}\left(m^{2}\right) /\left[(1-x) \Lambda_{a}^{2}\right]}\right]\right\}, \\
& h_{1}^{q(a)}(x)=\frac{g_{a}^{2}}{(2 \pi)^{2}} \frac{x}{2 \Lambda_{a}^{2}(1-x)^{2}}\left\{\Lambda_{a}^{2}(1-x)\left[e^{-2 L_{a}^{2}\left(m^{2}\right) /\left[(1-x) \Lambda_{a}^{2}\right]}-\Gamma\left(0,2 \frac{L_{a}^{2}\left(m^{2}\right)}{(1-x) \Lambda_{a}^{2}}\right)\right]\right. \\
& \left.-2 L_{a}^{2}\left(m^{2}\right) \Gamma\left(0,2 \frac{L_{a}^{2}\left(m^{2}\right)}{(1-x) \Lambda_{a}^{2}}\right)\right\} .
\end{aligned}
$$

\section{Axial-vector diquark including also longitudinal polarization}

The unintegrated parton densities are

$$
\begin{aligned}
f_{1}^{q(a)}\left(x, \boldsymbol{p}_{T}^{2}\right)= & \frac{g_{a}^{2}}{(2 \pi)^{3}} \frac{1}{4\left[\boldsymbol{p}_{T}^{2}+L_{a}^{2}\left(m^{2}\right)\right]^{2} M_{a}^{2}(1-x)}\left[\boldsymbol{p}_{T}^{4}+x M_{a}^{2}\left(2 \boldsymbol{p}_{T}^{2}+x M_{a}^{2}\right)\right. \\
& \left.+(1-x)^{2}\left[\boldsymbol{p}_{T}^{2}\left(M^{2}+m^{2}+2 M_{a}^{2}\right)+2 m^{2} M_{a}^{2}+6 x m M M_{a}^{2}+2 x^{2} M^{2} M_{a}^{2}+m^{2} M^{2}(1-x)^{2}\right]\right] \\
g_{1 L}^{q(a)}\left(x, \boldsymbol{p}_{T}^{2}\right)= & \frac{g_{a}^{2}}{(2 \pi)^{3}} \frac{1}{4\left[\boldsymbol{p}_{T}^{2}+L_{a}^{2}\left(m^{2}\right)\right]^{2} M_{a}^{2}(1-x)}\left[\boldsymbol{p}_{T}^{4}+x M_{a}^{2}\left(2 \boldsymbol{p}_{T}^{2}+x M_{a}^{2}\right)\right. \\
+ & \left.(1-x)^{2}\left[\boldsymbol{p}_{T}^{2}\left(2 M_{a}^{2}-m^{2}-M^{2}-4 m M\right)-2 m^{2} M_{a}^{2}-2 x m M M_{a}^{2}-2 x^{2} M^{2} M_{a}^{2}+m^{2} M^{2}(1-x)^{2}\right]\right], \\
g_{1 T}^{q(a)}\left(x, \boldsymbol{p}_{T}^{2}\right)= & \frac{g_{a}^{2} M}{(2 \pi)^{3}} \frac{(m+M) \boldsymbol{p}_{T}^{2}-m M(m+M)(1-x)^{2}+x M_{a}^{2}[M(2 x-1)+m]}{2\left[\boldsymbol{p}_{T}^{2}+L_{a}^{2}\left(m^{2}\right)\right]^{2} M_{a}^{2}}
\end{aligned}
$$




$$
\begin{aligned}
h_{1 L}^{\perp q(a)}\left(x, \boldsymbol{p}_{T}^{2}\right) & =\frac{g_{a}^{2} M}{(2 \pi)^{3}} \frac{(m+M) \boldsymbol{p}_{T}^{2}-m M(m+M)(1-x)^{2}+x M M_{a}^{2}-m M_{a}^{2}(x-2)}{2\left[\boldsymbol{p}_{T}^{2}+L_{a}^{2}\left(m^{2}\right)\right]^{2} M_{a}^{2}}, \\
h_{1 T}^{q(a)}\left(x, \boldsymbol{p}_{T}^{2}\right) & =-\frac{g_{a}^{2}}{(2 \pi)^{3}} \frac{\boldsymbol{p}_{T}^{4}+\left[2 x M_{a}^{2}+\left(m^{2}+M^{2}\right)(1-x)^{2}\right] \boldsymbol{p}_{T}^{2}+\left[x M_{a}^{2}+m M(1-x)^{2}\right]^{2}}{4\left[\boldsymbol{p}_{T}^{2}+L_{a}^{2}\left(m^{2}\right)\right]^{2} M_{a}^{2}(1-x)}, \\
h_{1 T}^{\perp q(a)}\left(x, \boldsymbol{p}_{T}^{2}\right) & =\frac{g_{a}^{2}}{(2 \pi)^{3}} \frac{M^{2}(m+M)^{2}(1-x)}{2\left[\boldsymbol{p}_{T}^{2}+L_{a}^{2}\left(m^{2}\right)\right]^{2} M_{a}^{2}}, \\
h_{1}^{q(a)}\left(x, \boldsymbol{p}_{T}^{2}\right) & =-\frac{g_{a}^{2}}{(2 \pi)^{3}} \frac{\boldsymbol{p}_{T}^{4}+\left[2 x M_{a}^{2}-2 m M(1-x)^{2}\right] \boldsymbol{p}_{T}^{2}+\left[x M_{a}^{2}+m M(1-x)^{2}\right]^{2}}{4 M_{a}^{2}(1-x)\left[\boldsymbol{p}_{T}^{2}+x M_{a}^{2}+(1-x)\left(m^{2}-x M^{2}\right)\right]^{2}} .
\end{aligned}
$$

The above results are valid for a point-like nucleon-quark-diquark coupling. For the other form factors it is sufficient to apply the replacements in Eqs. (A8) and (A9).

The integrated results are

- Point-like coupling (to avoid divergences we assume that the $\boldsymbol{p}_{T}^{2}$ integration is extended up to a finite cutoff $\Lambda_{a}^{2}$ )

$$
\begin{aligned}
& f_{1}^{q(a)}(x)=\frac{g_{a}^{2}}{(2 \pi)^{2}} \frac{1}{8 L_{a}^{2}\left(m^{2}\right)\left[\Lambda_{a}^{2}+L_{a}^{2}\left(m^{2}\right)\right] M_{a}^{2}(1-x)}\left[\Lambda_{a}^{2}\left[L_{a}^{4}\left(m^{2}\right)+\Lambda_{a}^{2} L_{a}^{2}\left(m^{2}\right)\right]\right. \\
& +(1-x)^{2}\left[2 M_{a}^{2}\left[m^{2}-L_{a}^{2}\left(m^{2}\right)\right]+x\left[\left(m^{2}-M^{2}\right)^{2}-M_{a}^{2}\left(m^{2}-6 m M+M^{2}\right)\right]+x^{2} 2 M^{2} M_{a}^{2}\right] \\
& \left.+L_{a}^{2}\left(m^{2}\right)\left[\Lambda_{a}^{2}+L_{a}^{2}\left(m^{2}\right)\right](1-x) \log \left(\frac{\Lambda_{a}^{2}}{L_{a}^{2}\left(m^{2}\right)}+1\right)\left[(1+x)\left(m^{2}-M^{2}\right)-2 M_{a}^{2}(1-x)\right]\right], \\
& g_{1}^{q(a)}(x)=\frac{g_{a}^{2}}{(2 \pi)^{2}} \frac{1}{8 L_{a}^{2}\left(m^{2}\right)\left[\Lambda_{a}^{2}+L_{a}^{2}\left(m^{2}\right)\right] M M_{a}^{2} x(1-x)}\left\{x M L_{a}^{2}\left(m^{2}\right) \Lambda_{a}^{2}\left[\Lambda_{a}^{2}+L_{a}^{2}\left(m^{2}\right)\right]+\Lambda_{a}^{2}(1-x)^{2}\right. \\
& \times\left[L_{a}^{2}\left(m^{2}\right)\left[m M^{2}(2 x-1)+m^{2} M(x-2)-m^{3}+x M\left(M^{2}-2 M_{a}^{2}\right)\right]\right. \\
& +m^{5}(1-x)-m^{4} M(x-2)+m^{3} M_{a}^{2} x+m^{3} M^{2}\left(1-x^{2}\right)+m^{2} M^{3} x\left(x^{2}-2 x-1\right) \\
& \left.+m M^{2} M_{a}^{2} x+m M^{4} x\left(2 x^{2}-x-1\right)+x^{3} M^{3}\left(M^{2}-2 M_{a}^{2}\right)\right] \\
& +(1-x) L_{a}^{2}\left(m^{2}\right)\left[\Lambda_{a}^{2}+L_{a}^{2}\left(m^{2}\right)\right] \log \left(\frac{\Lambda_{a}^{2}}{L_{a}^{2}\left(m^{2}\right)}+1\right) \\
& \times\left[L_{a}^{2}\left(m^{2}\right)(m+M)-m^{3}(1-x)+m^{2} M\left(x^{2}-2 x-1\right)-x m\left(3 M^{2}(1-x)+M_{a}^{2}\right)\right. \\
& \left.\left.+x M\left(M_{a}^{2}(1-2 x)+2 x M^{2}\right)\right]\right\}, \\
& h_{1}^{q(a)}(x)=-\frac{g_{a}^{2}}{(2 \pi)^{2}} \frac{1}{8 L_{a}^{2}\left(m^{2}\right)\left[\Lambda_{a}^{2}+L_{a}^{2}\left(m^{2}\right)\right] M_{a}^{2}(1-x)}\left[L_{a}^{2}\left(m^{2}\right) \Lambda_{a}^{2}\left(L_{a}^{2}\left(m^{2}\right)+\Lambda_{a}^{2}\right)\right. \\
& +(1-x)^{2} \Lambda_{a}^{2}\left[(m+M)^{2} L_{a}^{2}\left(m^{2}\right)+x(m-M)^{2}\left((m+M)^{2}-M_{a}^{2}\right)\right] \\
& \left.-2 L_{a}^{2}\left(m^{2}\right)\left(L_{a}^{2}\left(m^{2}\right)+\Lambda_{a}^{2}\right)(m+M)(m-x M)(1-x) \log \left(\frac{\Lambda_{a}^{2}}{L_{a}^{2}\left(m^{2}\right)}+1\right)\right] .
\end{aligned}
$$

- Dipolar form factor

$$
\begin{aligned}
f_{1}^{q(a)}(x)= & \frac{g_{a}^{2}}{(2 \pi)^{2}} \frac{1-x}{48 L_{a}^{6}\left(\Lambda_{a}^{2}\right) M_{a}^{2}}\left[2 L_{a}^{4}\left(\Lambda_{a}^{2}\right)+2 x M_{a}^{2}\left(L_{a}^{2}\left(\Lambda_{a}^{2}\right)+x M_{a}^{2}\right)\right. \\
& \left.+(1-x)^{2}\left[\left(2 M_{a}^{2}+m^{2}+M^{2}\right) L_{a}^{2}\left(\Lambda_{a}^{2}\right)+4 m^{2} M_{a}^{2}+x 12 m M M_{a}^{2}+x^{2} 4 M^{2} M_{a}^{2}+2 m^{2} M^{2}(1-x)^{2}\right]\right]
\end{aligned}
$$




$$
\begin{aligned}
g_{1}^{q(a)}(x)= & \frac{g_{a}^{2}}{(2 \pi)^{2}} \frac{1-x}{48 L_{a}^{6}\left(\Lambda_{a}^{2}\right) M_{a}^{2}}\left[2 L_{a}^{4}\left(\Lambda_{a}^{2}\right)+2 x M_{a}^{2}\left(L_{a}^{2}\left(\Lambda_{a}^{2}\right)+x M_{a}^{2}\right)\right. \\
& +(1-x)^{2}\left[\left(2 M_{a}^{2}-m^{2}-M^{2}-4 m M\right) L_{a}^{2}\left(\Lambda_{a}^{2}\right)-4 m^{2} M_{a}^{2}-x 4 m M M_{a}^{2}-x^{2} 4 M^{2} M_{a}^{2}\right. \\
& \left.\left.+2 m^{2} M^{2}(1-x)^{2}\right]\right], \\
h_{1}^{q(a)}(x)= & -\frac{g_{a}^{2}}{(2 \pi)^{2}} \frac{1-x}{24 L_{a}^{6}\left(\Lambda_{a}^{2}\right) M_{a}^{2}}\left[L_{a}^{4}\left(\Lambda_{a}^{2}\right)+\left[x M_{a}^{2}-m M(1-x)^{2}\right] L_{a}^{2}\left(\Lambda_{a}^{2}\right)+\left[x M_{a}^{2}+m M(1-x)^{2}\right]^{2}\right] .
\end{aligned}
$$

- Exponential form factor

$$
\begin{aligned}
& f_{1}^{q(a)}(x)=\frac{g_{a}^{2}}{(2 \pi)^{2}} \frac{1}{16 L_{a}^{2}\left(m^{2}\right) \Lambda_{a}^{2} M_{a}^{2}(1-x)^{2}} \\
& \times\left\{2\left[\Lambda_{a}^{2}(1-x) e^{-2\left[p_{T}^{2}+L_{a}^{2}\left(m^{2}\right)\right] /\left[(1-x) \Lambda_{a}^{2}\right]}-2 L_{a}^{2}\left(m^{2}\right) \Gamma\left(0, \frac{2 L_{a}^{2}\left(m^{2}\right)}{(1-x) \Lambda_{a}^{2}}\right)\right]\right. \\
& \times\left[(1-x)^{2}\left[m^{2}\left(2 M_{a}^{2}+M^{2}(1-x)^{2}\right)-L_{a}^{2}\left(m^{2}\right)\left(2 M_{a}^{2}+m^{2}+M^{2}\right)+2 x M M_{a}^{2}(x M+3 m)\right]\right. \\
& \left.+(1-x) x M_{a}^{2}\left(x M^{2}-m^{2}\right)+L_{a}^{2}\left(m^{2}\right)\left(L_{a}^{2}\left(m^{2}\right)-x M_{a}^{2}\right)\right] \\
& +\Lambda_{a}^{2} L_{a}^{2}\left(m^{2}\right)(1-x)\left[(1-x)^{2} 2 \Gamma\left(0, \frac{2 L_{a}^{2}\left(m^{2}\right)}{(1-x) \Lambda_{a}^{2}}\right)\left(m^{2}+M^{2}+2 M_{a}^{2}\right)\right. \\
& \left.\left.+(1-x) \Lambda_{a}^{2} e^{-2\left[\boldsymbol{p}_{T}^{2}+L_{a}^{2}\left(m^{2}\right)\right] /\left[(1-x) \Lambda_{a}^{2}\right]}+4 \Gamma\left(0, \frac{2 L_{a}^{2}\left(m^{2}\right)}{(1-x) \Lambda_{a}^{2}}\right)\left[x M_{a}^{2}-L_{a}^{2}\left(m^{2}\right)\right]\right]\right\} \\
& g_{1}^{q(a)}(x)=\frac{g_{a}^{2}}{(2 \pi)^{2}} \frac{1}{16 L_{a}^{2}\left(m^{2}\right) \Lambda_{a}^{2} M_{a}^{2}(1-x)^{2}} \\
& \times\left\{\left[\Lambda_{a}^{2}(1-x) e^{-2\left[\boldsymbol{p}_{T}^{2}+L_{a}^{2}\left(m^{2}\right)\right] /\left[(1-x) \Lambda_{a}^{2}\right]}-2 L_{a}^{2}\left(m^{2}\right) \Gamma\left(0, \frac{2 L_{a}^{2}\left(m^{2}\right)}{(1-x) \Lambda_{a}^{2}}\right)\right]\right. \\
& \times\left[(1-x)^{4} 2 m^{2} M^{2}-(1-x)^{2}\left[2 m M\left[x M_{a}^{2}-L_{a}^{2}\left(m^{2}\right)\right]-L_{a}^{2}\left(m^{2}\right)(m+M)^{2}\right]\right. \\
& +(1-x)\left[\Lambda_{a}^{2} L_{a}^{2}\left(m^{2}\right)+2 M_{a}^{2}\left(m^{2}(x-2)+(2 x-1) x^{2} M^{2}\right)\right] \\
& \left.+2 L_{a}^{2}\left(m^{2}\right)\left[L_{a}^{2}\left(m^{2}\right)-x M_{a}^{2}-2 M_{a}^{2}(1-x)^{2}\right]\right] \\
& \left.-2 L_{a}^{2}\left(m^{2}\right) \Lambda_{a}^{2}(1-x) \Gamma\left(0, \frac{2 L_{a}^{2}\left(m^{2}\right)}{(1-x) \Lambda_{a}^{2}}\right)\left[L_{a}^{2}\left(m^{2}\right)-2 x M_{a}^{2}+(1-x)^{2}\left[(m+M)^{2}+2 m M-2 M_{a}^{2}\right]\right]\right\}, \\
& h_{1}^{q(a)}(x)=\frac{g_{a}^{2}}{(2 \pi)^{2}} \frac{1}{16 L_{a}^{2}\left(m^{2}\right) \Lambda_{a}^{2} M_{a}^{2}(1-x)^{2}}\left\{\left[\Lambda_{a}^{2}(1-x)\left[\sinh \left(2 \frac{\boldsymbol{p}_{T}^{2}+L_{a}^{2}\left(m^{2}\right)}{(1-x) \Lambda_{a}^{2}}\right)-\cosh \left(2 \frac{\boldsymbol{p}_{T}^{2}+L_{a}^{2}\left(m^{2}\right)}{(1-x) \Lambda_{a}^{2}}\right)\right]\right.\right. \\
& \left.+2 L_{a}^{2}\left(m^{2}\right) \Gamma\left(0, \frac{2 L_{a}^{2}\left(m^{2}\right)}{(1-x) \Lambda_{a}^{2}}\right)\right]\left[(1-x)^{4} 2 m^{2} M^{2}+(1-x)^{2} 4 m M\left[x M_{a}^{2}+L_{a}^{2}\left(m^{2}\right)\right]\right. \\
& \left.+(1-x)\left[\Lambda_{a}^{2} L_{a}^{2}\left(m^{2}\right)+2 x M_{a}^{2}\left(x M^{2}-m^{2}\right)\right]+2 L_{a}^{2}\left(m^{2}\right)\left(L_{a}^{2}\left(m^{2}\right)-x M_{a}^{2}\right)\right] \\
& \left.+2 L_{a}^{2}\left(m^{2}\right) \Lambda_{a}^{2}(1-x) \Gamma\left(0, \frac{2 L_{a}^{2}\left(m^{2}\right)}{(1-x) \Lambda_{a}^{2}}\right)\left[L_{a}^{2}\left(m^{2}\right)-2 x M_{a}^{2}+(1-x)^{2} 2 m M\right]\right\} .
\end{aligned}
$$




\section{Axial-vector diquark including also time-like polarization}

The unintegrated parton densities are

$$
\begin{aligned}
f_{1}^{q(a)}\left(x, \boldsymbol{p}_{T}^{2}\right) & =\frac{g_{a}^{2}}{(2 \pi)^{3}} \frac{\left[\boldsymbol{p}_{T}^{2}+(m+x M)^{2}+2 m M x\right](1-x)}{2\left[\boldsymbol{p}_{T}^{2}+L_{a}^{2}\left(m^{2}\right)\right]^{2}} \\
g_{1 L}^{q(a)}\left(x, \boldsymbol{p}_{T}^{2}\right) & =-\frac{g_{a}^{2}}{(2 \pi)^{3}} \frac{\left[-\boldsymbol{p}_{T}^{2}+m^{2}+x^{2} M^{2}\right](1-x)}{2\left[\boldsymbol{p}_{T}^{2}+L_{a}^{2}\left(m^{2}\right)\right]^{2}} \\
g_{1 T}^{q(a)}\left(x, \boldsymbol{p}_{T}^{2}\right) & =-\frac{g_{a}^{2}}{(2 \pi)^{3}} \frac{M^{2} x(1-x)}{\left[\boldsymbol{p}_{T}^{2}+L_{a}^{2}\left(m^{2}\right)\right]^{2}}, \\
h_{1 L}^{\perp q(a)}\left(x, \boldsymbol{p}_{T}^{2}\right) & =\frac{g_{a}^{2}}{(2 \pi)^{3}} \frac{m M(1-x)}{\left[\boldsymbol{p}_{T}^{2}+L_{a}^{2}\left(m^{2}\right)\right]^{2}}, \\
h_{1 T}^{q(a)}\left(x, \boldsymbol{p}_{T}^{2}\right) & =-x h_{1 L}^{\perp q(a)}\left(x, \boldsymbol{p}_{T}^{2}\right), \\
h_{1 T}^{\perp q(a)}\left(x, \boldsymbol{p}_{T}^{2}\right) & =0, \\
h_{1}^{q(a)}\left(x, \boldsymbol{p}_{T}^{2}\right) & \equiv h_{1 T}^{q(a)}\left(x, \boldsymbol{p}_{T}^{2}\right) .
\end{aligned}
$$

The above results are valid for a point-like nucleon-quark-diquark coupling. For the other form factors it is sufficient to apply the replacements in Eqs. (A8) and (A9). The result for $f_{1}$ with dipolar form factor corresponds to that obtained in Ref. [46].

The integrated results are

- Point-like coupling (to avoid divergences we assume that the $\boldsymbol{p}_{T}^{2}$ integration is extended up to a finite cutoff $\Lambda_{a}^{2}$ )

$$
\begin{aligned}
& f_{1}^{q(a)}(x)=\frac{g_{a}^{2}(1-x)}{(2 \pi)^{2}} \frac{\left[(m+M)^{2}+2 m M-M_{a}^{2}\right] x \Lambda_{a}^{2}+L_{a}^{2}\left(m^{2}\right)\left[\Lambda_{a}^{2}+L_{a}^{2}\left(m^{2}\right)\right] \log \left(\frac{\Lambda_{a}^{2}}{L_{a}^{2}\left(m^{2}\right)}+1\right)}{4 L_{a}^{2}\left(m^{2}\right)\left[\Lambda_{a}^{2}+L_{a}^{2}\left(m^{2}\right)\right]} \\
& g_{1}^{q(a)}(x)=-\frac{g_{a}^{2}(1-x)}{(2 \pi)^{2}} \frac{\Lambda_{a}^{2}\left(L_{a}^{2}\left(m^{2}\right)+m^{2}+x^{2} M^{2}\right)-L_{a}^{2}\left(m^{2}\right)\left[\Lambda_{a}^{2}+L_{a}^{2}\left(m^{2}\right)\right] \log \left(\frac{\Lambda_{a}^{2}}{L_{a}^{2}\left(m^{2}\right)}+1\right)}{4 L_{a}^{2}\left(m^{2}\right)\left[\Lambda_{a}^{2}+L_{a}^{2}\left(m^{2}\right)\right]}, \\
& h_{1}^{q(a)}(x)=-\frac{g_{a}^{2}(1-x) \Lambda_{a}^{2}}{(2 \pi)^{2}} \frac{x m M}{2 L_{a}^{2}\left(m^{2}\right)\left[\Lambda_{a}^{2}+L_{a}^{2}\left(m^{2}\right)\right]} .
\end{aligned}
$$

- Dipolar form factor

$$
\begin{aligned}
& f_{1}^{q(a)}(x)=\frac{g_{a}^{2}}{(2 \pi)^{2}} \frac{\left[L_{a}^{2}\left(\Lambda_{a}^{2}\right)+2\left[(m+x M)^{2}+2 x m M\right]\right](1-x)^{3}}{24 L_{a}^{6}\left(\Lambda_{a}^{2}\right)} \\
& g_{1}^{q(a)}(x)=\frac{g_{a}^{2}}{(2 \pi)^{2}} \frac{\left[L_{a}^{2}\left(\Lambda_{a}^{2}\right)-2\left(m^{2}+x^{2} M^{2}\right)\right](1-x)^{3}}{24 L_{a}^{6}\left(\Lambda_{a}^{2}\right)} \\
& h_{1}^{q(a)}(x)=-\frac{g_{a}^{2}}{(2 \pi)^{2}} \frac{m M x(1-x)^{3}}{6 L_{a}^{6}\left(\Lambda_{a}^{2}\right)}
\end{aligned}
$$

- Exponential form factor

$$
\begin{gathered}
f_{1}^{q(a)}(x)=\frac{g_{a}^{2}}{(2 \pi)^{2}} \frac{1}{4}\left\{e^{-2 L_{a}^{2}\left(m^{2}\right) /\left[(1-x) \Lambda_{a}^{2}\right]} \frac{\left[(m+x M)^{2}+2 m x M-L_{a}^{2}\left(m^{2}\right)\right](1-x)}{L_{a}^{2}\left(m^{2}\right)}\right. \\
\left.-\Gamma\left(0,2 \frac{L_{a}^{2}\left(m^{2}\right)}{(1-x) \Lambda_{a}^{2}}\right) \frac{2\left[(m+x M)^{2}+2 m x M-L_{a}^{2}\left(m^{2}\right)\right]-(1-x) \Lambda_{a}^{2}}{\Lambda_{a}^{2}}\right\}, \\
g_{1}^{q(a)}(x)=\frac{g_{a}^{2}}{(2 \pi)^{2}} \frac{1}{4}\left\{\Gamma\left(0,2 \frac{L_{a}^{2}\left(m^{2}\right)}{(1-x) \Lambda_{a}^{2}}\right) \frac{2\left[m^{2}+x^{2} M^{2}+L_{a}^{2}\left(m^{2}\right)\right]+(1-x) \Lambda_{a}^{2}}{\Lambda_{a}^{2}}\right.
\end{gathered}
$$




$$
\begin{gathered}
\left.-e^{-2 L_{a}^{2}\left(m^{2}\right) /\left[(1-x) \Lambda_{a}^{2}\right]} \frac{(1-x)\left[m^{2}+x^{2} M^{2}+L_{a}^{2}\left(m^{2}\right)\right]}{L_{a}^{2}\left(m^{2}\right)}\right\}, \\
h_{1}^{q(a)}(x)=\frac{g_{a}^{2}}{(2 \pi)^{2}} \frac{m x M}{2}\left\{\frac{2}{\Lambda_{a}^{2}} \Gamma\left(0, \frac{2 L_{a}^{2}\left(m^{2}\right)}{(1-x) \Lambda_{a}^{2}}\right)-e^{-2 L_{a}^{2}\left(m^{2}\right) /\left[(1-x) \Lambda_{a}^{2}\right]} \frac{1-x}{L_{a}^{2}\left(m^{2}\right)}\right\} .
\end{gathered}
$$

\section{APPENDIX B: T-ODD FUNCTIONS IN DIFFERENT VARIATIONS OF THE MODEL}

As a continuation of App. $\mathrm{A}$, here we list the Sivers and Boer-Mulders functions, namely the leading-twist T-odd parton densities obtained in the context of our spectator diquark model, again for all the combinations of diquark propagators and nucleon-quark-diquark vertices.

\section{Scalar diquark}

For scalar diquarks, we have

$$
\begin{aligned}
& f_{1 T}^{\perp q(s)}\left(x, \boldsymbol{p}_{T}^{2}\right)=-\frac{g_{s}\left(p^{2}\right)}{4} \frac{1}{(2 \pi)^{3}} \frac{M e_{c}^{2}}{2(1-x) P^{+}} \frac{2 \operatorname{Im} J_{1}^{s}}{p^{2}-m^{2}} \\
& h_{1}^{\perp q(s)}\left(x, \boldsymbol{p}_{T}^{2}\right)=f_{1 T}^{\perp q(s)}\left(x, \boldsymbol{p}_{T}^{2}\right),
\end{aligned}
$$

where the $J_{1}^{s}$ integral is defined as

$$
\begin{aligned}
&\left(\varepsilon_{T}^{i j} p_{T i} S_{T j}\right) J_{1}^{s}=\int \frac{d^{4} l}{(2 \pi)^{4}} \frac{g_{s}\left((p-l)^{2}\right)}{\left(D_{1}+i \varepsilon\right)\left(D_{2}-i \varepsilon\right)\left(D_{3}+i \varepsilon\right)\left(D_{4}+i \varepsilon\right)} \operatorname{Tr}\left[(\not p-\not+m)(\not P+M) \gamma_{5} \not(\not p+m)(2 P-2 p+l)_{\rho} n_{-}^{\rho} \gamma^{+}\right] \\
&=\int \frac{d^{4} l}{(2 \pi)^{4}} \frac{g_{s}\left((p-l)^{2}\right)}{\left(D_{1}+i \varepsilon\right)\left(D_{2}-i \varepsilon\right)\left(D_{3}+i \varepsilon\right)\left(D_{4}+i \varepsilon\right)} 4 i\left(l^{+}+2(1-x) P^{+}\right) \\
&\left(l^{+} M \varepsilon_{T}^{i j} p_{T i} S_{T j}-P^{+}(m+x M) \varepsilon_{T}^{i j} l_{T i} S_{T j}\right),
\end{aligned}
$$

with $D_{1}, D_{2}, D_{3}, D_{4}$ defined in Eq. (85). The imaginary part of $J_{1}^{s}$ can be extracted by using the Cutkosky cutting rules on the loop diagram of Fig. 3, which in the present case amount to put on shell the eikonalized virtual quark propagator $D_{2}$ and the virtual scalar diquark propagator $D_{4}$. The resulting $\delta$ functions (see below) reduce the integral in Eq. (B2) to a bidimensional integral in $d^{2} \boldsymbol{l}_{T}$. In general, for a $n$-dimensional integral $\int d^{n} l l_{\rho} f(l, p)$ the term $l_{\rho}$ can be replaced by the expression $p_{\rho}(l \cdot p) / p^{2}$. For the present case $n=2$ and with the identification $l_{\rho}=l_{T i}, p_{\rho}=p_{T i}$, we finally can write

$$
\begin{aligned}
2 \operatorname{Im} J_{1}^{s} & =\int \frac{d^{4} l}{(2 \pi)^{4}} \frac{g_{s}\left((p-l)^{2}\right)}{D_{1} D_{3}} 4\left(l^{+}+2(1-x) P^{+}\right)\left(l^{+} M-P^{+}(m+x M) \frac{\boldsymbol{l}_{T} \cdot \boldsymbol{p}_{T}}{\boldsymbol{p}_{T}^{2}}\right)(2 \pi i) \delta\left(D_{2}\right)(-2 \pi i) \delta\left(D_{4}\right) \\
& =-4 P^{+}(m+x M)(1-x) g_{s} \mathcal{I}_{1} .
\end{aligned}
$$

The explicit expression of $\mathcal{I}_{1}$ clearly depends on the choice of the nucleon-quark-scalar diquark vertex form factor.

The Boer-Mulders calculation gives exactly the same results as the Sivers one, in the scalar diquark framework, since the relevant trace over Dirac-Lorentz structures is now given by

$\operatorname{Tr}\left[(\not p-\not l+m)(\not P+M)(\not p+m)(2 P-2 p+l)_{\rho} n_{-}^{\rho} i \sigma^{i+} \gamma_{5}\right]=-4 i\left(l^{+}+2(1-x) P^{+}\right)\left(l^{+} M \varepsilon_{T}^{i j} p_{T j}-P^{+}(m+x M) \varepsilon_{T}^{i j} l_{T j}\right)$.

- Point-like coupling

$$
\begin{aligned}
2 \operatorname{Im} J_{1}^{s} & =g_{s} \int \frac{d^{4} l}{(2 \pi)^{4}} \frac{1}{D_{1} D_{3}} 4\left(l^{+}+2(1-x) P^{+}\right)\left(l^{+} M-P^{+}(m+x M) \frac{\boldsymbol{l}_{T} \cdot \boldsymbol{p}_{T}}{\boldsymbol{p}_{T}^{2}}\right)(2 \pi i) \delta\left(D_{2}\right)(-2 \pi i) \delta\left(D_{4}\right) \\
& =-4 P^{+}(m+x M)(1-x) g_{s} \mathcal{I}_{1}^{p . l .}=-g_{s} \frac{P^{+}(m+x M)(1-x)}{\pi \boldsymbol{p}_{T}^{2}} \log \left(\frac{L_{s}^{2}\left(m^{2}\right)+\boldsymbol{p}_{T}^{2}}{L_{s}^{2}\left(m^{2}\right)}\right),
\end{aligned}
$$


where $\mathcal{I}_{1}^{p . l .}$ is calculated in App. C.

Using Eq. (8), the final result is then

$$
\begin{aligned}
& f_{1 T}^{\perp q(s)}\left(x, \boldsymbol{p}_{T}^{2}\right)=-\frac{g_{s}^{2}}{4} \frac{M e_{c}^{2}}{(2 \pi)^{4}} \frac{(m+x M)(1-x)}{\left.\boldsymbol{p}_{T}^{2}\left[L_{s}^{2}\left(m^{2}\right)+\boldsymbol{p}_{T}^{2}\right)\right]} \log \left(\frac{L_{s}^{2}\left(m^{2}\right)+\boldsymbol{p}_{T}^{2}}{L_{s}^{2}\left(m^{2}\right)}\right) \\
& h_{1}^{\perp q(s)}\left(x, \boldsymbol{p}_{T}^{2}\right)=f_{1 T}^{\perp q(s)}\left(x, \boldsymbol{p}_{T}^{2}\right) .
\end{aligned}
$$

- Dipolar form factor. The final results, already given in Eq. (98) and (100), are

$$
\begin{aligned}
& f_{1 T}^{\perp q(s)}\left(x, \boldsymbol{p}_{T}^{2}\right)=-\frac{g_{s}^{2}}{4} \frac{M e_{c}^{2}}{(2 \pi)^{4}} \frac{(1-x)^{3}(m+x M)}{L_{s}^{2}\left(\Lambda_{s}^{2}\right)\left[\boldsymbol{p}_{T}^{2}+L_{s}^{2}\left(\Lambda_{s}^{2}\right)\right]^{3}} \\
& h_{1}^{\perp q(s)}\left(x, \boldsymbol{p}_{T}^{2}\right)=f_{1 T}^{\perp q(s)}\left(x, \boldsymbol{p}_{T}^{2}\right)
\end{aligned}
$$

- Exponential form factor

$$
\begin{aligned}
2 \operatorname{Im} J_{1}^{s} & =g_{s} \int \frac{d^{4} l}{(2 \pi)^{4}} \frac{e^{\left[(p-l)^{2}-m^{2}\right] / \Lambda_{s}^{2}}}{D_{1} D_{3}} 4\left(l^{+}+2(1-x) P^{+}\right)\left(l^{+} M-P^{+}(m+x M) \frac{\boldsymbol{l}_{T} \cdot \boldsymbol{p}_{T}}{\boldsymbol{p}_{T}^{2}}\right)(2 \pi i) \delta\left(D_{2}\right)(-2 \pi i) \delta\left(D_{4}\right) \\
& =-4 P^{+}(m+x M)(1-x) g_{s} \mathcal{I}_{1}^{e x p} \\
& =-g_{s} \frac{P^{+}(m+x M)(1-x)}{\pi \boldsymbol{p}_{T}^{2}}\left[\Gamma\left(0, \frac{L_{s}^{2}\left(m^{2}\right)}{(1-x) \Lambda_{s}^{2}}\right)-\Gamma\left(0, \frac{L_{s}^{2}\left(m^{2}\right)+\boldsymbol{p}_{T}^{2}}{(1-x) \Lambda_{s}^{2}}\right)\right]
\end{aligned}
$$

and $\mathcal{I}_{1}^{e x p}$ is calculated in App. C.

The final results are then

$$
\begin{aligned}
f_{1 T}^{\perp q(s)}\left(x, \boldsymbol{p}_{T}^{2}\right)=- & \frac{g_{s}^{2}}{4} \frac{M e_{c}^{2}}{(2 \pi)^{4}} \frac{(m+x M)(1-x)}{\boldsymbol{p}_{T}^{2}\left[L_{s}^{2}\left(m^{2}\right)+\boldsymbol{p}_{T}^{2}\right]} e^{-\left[\boldsymbol{p}_{T}^{2}+L_{s}^{2}\left(m^{2}\right)\right] /\left[(1-x) \Lambda_{s}^{2}\right]} \\
& \times\left[\Gamma\left(0, \frac{L_{s}^{2}\left(m^{2}\right)}{(1-x) \Lambda_{s}^{2}}\right)-\Gamma\left(0, \frac{L_{s}^{2}\left(m^{2}\right)+\boldsymbol{p}_{T}^{2}}{(1-x) \Lambda_{s}^{2}}\right)\right], \\
h_{1}^{\perp q(s)}\left(x, \boldsymbol{p}_{T}^{2}\right)= & f_{1 T}^{\perp q(s)}\left(x, \boldsymbol{p}_{T}^{2}\right) .
\end{aligned}
$$

\section{Axial-vector diquark with light-cone transverse polarization only}

We have

$$
\begin{aligned}
& f_{1 T}^{\perp q(a)}\left(x, \boldsymbol{p}_{T}^{2}\right)=\frac{g_{a}\left(p^{2}\right)}{4} \frac{1}{(2 \pi)^{3}} \frac{M e_{c}^{2}}{4(1-x) P^{+}} \frac{2 \operatorname{Im} J_{1}^{a}}{p^{2}-m^{2}} \\
& h_{1}^{\perp q(a)}\left(x, \boldsymbol{p}_{T}^{2}\right)=\frac{g_{a}\left(p^{2}\right)}{4} \frac{1}{(2 \pi)^{3}} \frac{M e_{c}^{2}}{4(1-x) P^{+}} \frac{2 \operatorname{Im} J_{1}^{\prime(a)}}{p^{2}-m^{2}},
\end{aligned}
$$

where now the $J_{1}^{a}$ and $J_{1}^{\prime a}$ integrals are defined as

$$
\begin{aligned}
\left(\varepsilon_{T}^{i j} p_{T i} S_{T j}\right) J_{1}^{a}=\int \frac{d^{4} l}{(2 \pi)^{4}} \frac{g_{a}\left((p-l)^{2}\right)}{\left(D_{1}+i \varepsilon\right)\left(D_{2}-i \varepsilon\right)\left(D_{3}+i \varepsilon\right)\left(D_{4}+i \varepsilon\right)} \\
\operatorname{Tr}\left[(\not p-\not+m) \gamma^{\mu} \gamma_{5}(\not P+M) \gamma_{5} \$ \gamma^{\alpha} \gamma_{5}(\not p+m) d_{\mu \nu}(p-l-P) d_{\sigma \alpha}(P-p)\right. \\
\left.\quad\left[(2 P-2 p+l)_{\rho} g^{\nu \sigma}-\left(P-p+\left(1+\kappa_{a}\right) l\right)^{\sigma} g_{\rho}^{\nu}-\left(P-p-\kappa_{a} l\right)^{\nu} g_{\rho}^{\sigma}\right] n_{-}^{\rho} \gamma^{+}\right]
\end{aligned}
$$

and

$$
\begin{aligned}
&\left(-\varepsilon_{T}^{i j} p_{T j}\right) J_{1}^{\prime a}=\int \frac{d^{4} l}{(2 \pi)^{4}} \frac{g_{a}\left((p-l)^{2}\right)}{\left(D_{1}+i \varepsilon\right)\left(D_{2}-i \varepsilon\right)\left(D_{3}+i \varepsilon\right)\left(D_{4}+i \varepsilon\right)} \\
& \operatorname{Tr}\left[(\not p-\not+m) \gamma^{\mu} \gamma_{5}(\not P+M) \gamma^{\alpha} \gamma_{5}(\not p+m) d_{\mu \nu}(p-l-P) d_{\sigma \alpha}(P-p)\right. \\
& {\left.\left[(2 P-2 p+l)_{\rho} g^{\nu \sigma}-\left(P-p+\left(1+\kappa_{a}\right) l\right)^{\sigma} g_{\rho}^{\nu}-\left(P-p-\kappa_{a} l\right)^{\nu} g_{\rho}^{\sigma}\right] n_{-}^{\rho} i \sigma^{i+} \gamma_{5}\right], }
\end{aligned}
$$


and the explicit expressions of the $d_{\mu \nu}(p-l-P)$ and $d_{\sigma \alpha}(P-p)$ structures are those expressed in the first line in Eq. (10).

- Point-like coupling (to avoid divergences we assume that the $\boldsymbol{p}_{T}^{2}$ integration is extended up to a finite cutoff $\Lambda_{a}^{2}$ )

$$
\begin{aligned}
2 \operatorname{Im} J_{1}^{a} & =-8 P^{+} x(m+x M) g_{a} \mathcal{I}_{1}^{p . l .}=-g_{a} \frac{2 P^{+} x(m+x M)}{\pi \boldsymbol{p}_{T}^{2}} \log \left(\frac{L_{a}^{2}\left(m^{2}\right)+\boldsymbol{p}_{T}^{2}}{L_{a}^{2}\left(m^{2}\right)}\right) \\
2 \operatorname{Im} J_{1}^{\prime a} & =8 P^{+}(m+x M) g_{a} \mathcal{I}_{1}^{p . l .}=g_{a} \frac{2 P^{+}(m+x M)}{\pi \boldsymbol{p}_{T}^{2}} \log \left(\frac{L_{a}^{2}\left(m^{2}\right)+\boldsymbol{p}_{T}^{2}}{L_{a}^{2}\left(m^{2}\right)}\right),
\end{aligned}
$$

where $\mathcal{I}_{1}^{p . l .}$ is the same integral as in Eq. (B4) but with the substitution $L_{s}\left(m^{2}\right) \leftrightarrow L_{a}\left(m^{2}\right)$.

Using again Eq. (8), the final result is

$$
\begin{aligned}
& f_{1 T}^{\perp q(a)}\left(x, \boldsymbol{p}_{T}^{2}\right)=\frac{g_{a}^{2}}{4} \frac{M e_{c}^{2}}{(2 \pi)^{4}} \frac{x(m+x M)}{\boldsymbol{p}_{T}^{2}\left[L_{a}^{2}\left(m^{2}\right)+\boldsymbol{p}_{T}^{2}\right]} \log \left(\frac{L_{a}^{2}\left(m^{2}\right)+\boldsymbol{p}_{T}^{2}}{L_{a}^{2}\left(m^{2}\right)}\right) \\
& h_{1}^{\perp q(a)}\left(x, \boldsymbol{p}_{T}^{2}\right)=-\frac{1}{x} f_{1 T}^{\perp q(a)}\left(x, \boldsymbol{p}_{T}^{2}\right) .
\end{aligned}
$$

- Dipolar form factor. The final results, already given in Eq. (99) and (101), are

$$
\begin{aligned}
& f_{1 T}^{\perp q(a)}\left(x, \boldsymbol{p}_{T}^{2}\right)=\frac{g_{a}^{2}}{4} \frac{M e_{c}^{2}}{(2 \pi)^{4}} \frac{(1-x)^{2} x(m+x M)}{L_{a}^{2}\left(\Lambda_{a}^{2}\right)\left[\boldsymbol{p}_{T}^{2}+L_{a}^{2}\left(\Lambda_{a}^{2}\right)\right]^{3}} \\
& h_{1}^{\perp q(a)}\left(x, \boldsymbol{p}_{T}^{2}\right)=-\frac{1}{x} f_{1 T}^{\perp q(a)}\left(x, \boldsymbol{p}_{T}^{2}\right) .
\end{aligned}
$$

- Exponential form factor

$$
\begin{aligned}
2 \operatorname{Im} J_{1}^{a} & =-8 P^{+} x(m+x M) g_{a} \mathcal{I}_{1}^{\text {exp }} \\
& =-g_{a} \frac{2 P^{+} x(m+x M)}{\pi \boldsymbol{p}_{T}^{2}}\left[\Gamma\left(0, \frac{L_{a}^{2}\left(m^{2}\right)}{(1-x) \Lambda_{a}^{2}}\right)-\Gamma\left(0, \frac{L_{a}^{2}\left(m^{2}\right)+\boldsymbol{p}_{T}^{2}}{(1-x) \Lambda_{a}^{2}}\right)\right], \\
2 \operatorname{Im} J_{1}^{\prime a} & =8 P^{+}(m+x M) g_{a} \mathcal{I}_{1}^{\text {exp }} \\
& =g_{a} \frac{2 P^{+}(m+x M)}{\pi \boldsymbol{p}_{T}^{2}}\left[\Gamma\left(0, \frac{L_{a}^{2}\left(m^{2}\right)}{(1-x) \Lambda_{a}^{2}}\right)-\Gamma\left(0, \frac{L_{a}^{2}\left(m^{2}\right)+\boldsymbol{p}_{T}^{2}}{(1-x) \Lambda_{a}^{2}}\right)\right] .
\end{aligned}
$$

The final result is, then,

$$
\begin{aligned}
f_{1 T}^{\perp q(a)}\left(x, \boldsymbol{p}_{T}^{2}\right)=\frac{g_{a}^{2}}{4} & \frac{M e_{c}^{2}}{(2 \pi)^{4}} \frac{x(m+x M)}{\boldsymbol{p}_{T}^{2}\left[L_{a}^{2}\left(m^{2}\right)+\boldsymbol{p}_{T}^{2}\right]} e^{-\left[\boldsymbol{p}_{T}^{2}+L_{a}^{2}\left(m^{2}\right)\right] /\left[(1-x) \Lambda_{a}^{2}\right]} \\
& \times\left[\Gamma\left(0, \frac{L_{a}^{2}\left(m^{2}\right)}{(1-x) \Lambda_{a}^{2}}\right)-\Gamma\left(0, \frac{L_{a}^{2}\left(m^{2}\right)+\boldsymbol{p}_{T}^{2}}{(1-x) \Lambda_{a}^{2}}\right)\right], \\
h_{1}^{\perp q(a)}\left(x, \boldsymbol{p}_{T}^{2}\right)= & -\frac{1}{x} f_{1 T}^{\perp q(a)}\left(x, \boldsymbol{p}_{T}^{2}\right) .
\end{aligned}
$$

\section{Axial-vector diquark including also longitudinal polarization}

We have

$$
\begin{aligned}
& f_{1 T}^{\perp q(a)}\left(x, \boldsymbol{p}_{T}^{2}\right)=\frac{g_{a}\left(p^{2}\right)}{4} \frac{1}{(2 \pi)^{3}} \frac{M e_{c}^{2}}{4(1-x) P^{+}} \frac{2 \operatorname{Im} J_{1}^{a}}{p^{2}-m^{2}} \\
& h_{1}^{\perp q(a)}\left(x, \boldsymbol{p}_{T}^{2}\right)=\frac{g_{a}\left(p^{2}\right)}{4} \frac{1}{(2 \pi)^{3}} \frac{M e_{c}^{2}}{4(1-x) P^{+}} \frac{2 \operatorname{Im} J_{1}^{\prime a}}{p^{2}-m^{2}}
\end{aligned}
$$

where the $J_{1}^{a}$ and $J_{1}^{\prime a}$ integrals are defined as in Eqs. (B9) and (B10), respectively, but now the second line in Eq. (10) is employed for the $d_{\mu \nu}(p-l-P)$ and $d_{\sigma \alpha}(P-p)$ Lorentz structures. 
- Point-like coupling (to avoid divergences we assume that the $\boldsymbol{p}_{T}^{2}$ integration is extended up to a finite cutoff $\Lambda_{a}^{2}$ )

$$
\begin{aligned}
& 2 \operatorname{Im} J_{1}^{a}=g_{a} \int \frac{d^{4} l}{(2 \pi)^{4}} \frac{1}{D_{1} D_{3}}(2 \pi i) \delta\left(D_{2}\right)(-2 \pi i) \delta\left(D_{4}\right) \\
& \times\left\{X\left(x, \boldsymbol{p}_{T}^{2}\right)\left(1+\frac{\boldsymbol{l}_{T}^{2}}{2 M_{a}^{2}}\right) \boldsymbol{l}_{T} \cdot\left(\boldsymbol{p}_{T}-\frac{1}{2} \boldsymbol{l}_{T}\right)+\right. \\
& \left(\frac{\boldsymbol{l}_{T} \cdot \boldsymbol{p}_{T}}{\boldsymbol{p}_{T}^{2}}\right)\left[Y_{1}\left(x, \boldsymbol{p}_{T}^{2}\right)+Y_{2}\left(x, \boldsymbol{p}_{T}^{2}\right)\left(\boldsymbol{l}_{T} \cdot \boldsymbol{p}_{T}\right)+Y_{3}\left(x, \boldsymbol{p}_{T}^{2}\right) \boldsymbol{l}_{T}^{2}\right]+ \\
& \left.W\left(x, \boldsymbol{p}_{T}^{2}\right)\left(\frac{\boldsymbol{l}_{T} \cdot \boldsymbol{S}_{T}}{\boldsymbol{S}_{T}^{2}}\right) \boldsymbol{S}_{T} \cdot\left[\boldsymbol{l}_{T}-2 \boldsymbol{p}_{T}\right]\right\}, \\
& 2 \operatorname{Im} J_{1}^{\prime a}=g_{a} \int \frac{d^{4} l}{(2 \pi)^{4}} \frac{1}{D_{1} D_{3}}(2 \pi i) \delta\left(D_{2}\right)(-2 \pi i) \delta\left(D_{4}\right) \\
& \times\left\{-X\left(x, \boldsymbol{p}_{T}^{2}\right)\left(1+\frac{\boldsymbol{l}_{T}^{2}}{2 M_{a}^{2}}\right) \boldsymbol{l}_{T} \cdot\left(\boldsymbol{p}_{T}-\frac{1}{2} \boldsymbol{l}_{T}\right)+\right. \\
& \left(\frac{\boldsymbol{l}_{T} \cdot \boldsymbol{p}_{T}}{\boldsymbol{p}_{T}^{2}}\right)\left[Y_{1}\left(x, \boldsymbol{p}_{T}^{2}\right)-Y_{2}\left(x, \boldsymbol{p}_{T}^{2}\right)\left(\boldsymbol{l}_{T} \cdot \boldsymbol{p}_{T}\right)-Y_{3}\left(x, \boldsymbol{p}_{T}^{2}\right) \boldsymbol{l}_{T}^{2}+\right. \\
& \left.\left.+2 M_{a}^{2}[(m+x M)(1-x)-x m]+M_{a}^{2}\left[m\left(\kappa_{a}-1\right)-M\left(1+\kappa_{a}\right) x\right] \boldsymbol{l}_{T}^{2}\right]\right\},
\end{aligned}
$$

where the integrals $X, W, Y_{i}, i=1-3$ are listed in App. C. Unfortunately, most of the above combinations are divergent under the $d \boldsymbol{l}_{T}$ integration. This is a typical pathology when choosing the point-like form factor for the nucleon-quark-diquark vertex, without any ad-hoc cut-off.

- Dipolar form factor

$$
\begin{aligned}
& 2 \operatorname{Im} J_{1}^{a}= g_{a} \int \frac{d^{4} l}{(2 \pi)^{4}} \frac{1}{D_{1}\left(D_{3}\right)^{2}}(2 \pi i) \delta\left(D_{2}\right)(-2 \pi i) \delta\left(D_{4}\right) \\
& \times\left\{X\left(x, \boldsymbol{p}_{T}^{2}\right)\left(1-\frac{1}{2} \boldsymbol{l}_{T}^{2}+\frac{\boldsymbol{l}_{T} \cdot \boldsymbol{p}_{T}}{\boldsymbol{p}_{T}^{2}} \frac{\boldsymbol{l}_{T}^{2}}{2 M_{a}^{2}}-\frac{\boldsymbol{l}_{T}^{4}}{4 M_{a}^{4}}\right)+\right. \\
& W\left(x, \boldsymbol{p}_{T}^{2}\right)\left(\frac{\boldsymbol{l}_{T} \cdot \boldsymbol{S}_{T}}{\boldsymbol{S}_{T}^{2}}\right) \boldsymbol{S}_{T} \cdot\left[\boldsymbol{l}_{T}-2 \boldsymbol{p}_{T}\right]+ \\
& 2 \operatorname{Im} J_{1}^{\prime a}= g_{a} \int \frac{d^{4} l}{(2 \pi)^{4}} \frac{1}{D_{1}\left(D_{3}\right)^{2}}(2 \pi i) \delta\left(D_{2}\right)(-2 \pi i) \delta\left(D_{4}\right) \\
& \times\left.\left\{-Y_{1}\left(x, \boldsymbol{p}_{T}^{2}\right)+Y_{2}\left(x, \boldsymbol{p}_{T}^{2}\right) \frac{\boldsymbol{l}_{T} \cdot \boldsymbol{p}_{T}}{\boldsymbol{p}_{T}^{2}}+Y_{3}\left(x, \boldsymbol{p}_{T}^{2}\right) \boldsymbol{l}_{T}^{2}\right]\right\}, \\
&\left(\frac{\boldsymbol{l}_{T} \cdot \boldsymbol{p}_{T}}{\boldsymbol{p}_{T}^{2}}\right)\left[1+\frac{\boldsymbol{l}_{T}^{2}}{2 M_{a}^{2}}\right) \boldsymbol{l}_{T} \cdot\left(\boldsymbol{p}_{T}\left(x, \boldsymbol{p}_{T}^{2}\right)-Y_{2}\left(x, \boldsymbol{p}_{T}^{2}\right) \boldsymbol{l}_{T} \cdot \boldsymbol{p}_{T}-Y_{3}\left(x, \boldsymbol{p}_{T}^{2}\right) \boldsymbol{l}_{T}^{2}+\right. \\
&\left.\left.2 M_{a}^{2}[(m+x M)(1-x)-m x]+M_{a}^{2}\left[m\left(\kappa_{a}-1\right)-M\left(1+\kappa_{a}\right) x\right] \boldsymbol{l}_{T}^{2}\right]\right\},
\end{aligned}
$$

where the integrals $X, W, Y_{i}, i=1-3$ are listed in App. C. Unfortunately, most of the above combinations are divergent under the $d \boldsymbol{l}_{T}$ integration, unless a dipolar form factor is considered with a higher degree, for example proportional to $\left[\boldsymbol{p}_{T}^{2}+L_{a}^{2}\left(\Lambda_{a}^{2}\right)\right]^{-3}$ in Eq. (13). This would introduce a $1 /\left(D_{3}\right)^{3}$ term inside Eq. (B18), instead of 
$1 /\left(D_{3}\right)^{2}$, and grant the convergence of the various integrals. With this very choice, we obtain

$$
\begin{aligned}
2 \operatorname{Im} J_{1}^{a}= & g_{a} \frac{(1-x)^{2}}{2 P^{+}}\left\{X\left(x, \boldsymbol{p}_{T}^{2}\right)\left[\boldsymbol{p}_{T}^{2} \mathcal{I}_{1}^{\prime d i p}+\left(\frac{\boldsymbol{p}_{T}^{2}}{2 M_{a}^{2}}-\frac{1}{2}\right) \mathcal{I}_{2}^{\prime d i p}-\frac{1}{4 M_{a}^{2}} \mathcal{I}_{5}^{\prime d i p}\right]+\right. \\
& Y_{1}\left(x, \boldsymbol{p}_{T}^{2}\right) \mathcal{I}_{1}^{\prime d i p}+Y_{2}\left(x, \boldsymbol{p}_{T}^{2}\right) \mathcal{I}_{3}^{\prime d i p}+Y_{3}\left(x, \boldsymbol{p}_{T}^{2}\right) \mathcal{I}_{2}^{\prime d i p}+ \\
& \left.W\left(x, \boldsymbol{p}_{T}^{2}\right)\left(\mathcal{I}_{7}^{\prime d i p}-2 \boldsymbol{p}_{T} \cdot \boldsymbol{S}_{T} \mathcal{I}_{6}^{\prime d i p}\right)\right\} \\
2 \operatorname{Im} J_{1}^{\prime a}= & g_{a} \frac{(1-x)^{2}}{2 P^{+}}\left\{X\left(x, \boldsymbol{p}_{T}^{2}\right)\left[-\boldsymbol{p}_{T}^{2} \mathcal{I}_{1}^{\prime d i p}+\left(\frac{1}{2}-\frac{\boldsymbol{p}_{T}^{2}}{2 M_{a}^{2}}\right) \mathcal{I}_{2}^{\prime d i p}+\frac{1}{4 M_{a}^{2}} \mathcal{I}_{5}^{\prime d i p}\right]+\right. \\
& Y_{1}\left(x, \boldsymbol{p}_{T}^{2}\right) \mathcal{I}_{1}^{\prime d i p}-Y_{2}\left(x, \boldsymbol{p}_{T}^{2}\right) \mathcal{I}_{3}^{\prime d i p}-Y_{3}\left(x, \boldsymbol{p}_{T}^{2}\right) \mathcal{I}_{2}^{\prime d i p}+ \\
& \left.2 M_{a}^{2}[(m+x M)(1-x)-m x] \mathcal{I}_{1}^{\prime d i p}+M_{a}^{2}\left[m\left(\kappa_{a}-1\right)-M\left(1+\kappa_{a}\right) x\right] \mathcal{I}_{2}^{\prime d i p}\right\},
\end{aligned}
$$

where the integrals $\mathcal{I}_{i}^{\prime d i p}, i=1-7$, are listed in App. C.

The final result is, then,

$$
\begin{aligned}
f_{1 T}^{\perp q(a)}\left(x, \boldsymbol{p}_{T}^{2}\right)= & -\frac{g_{a}^{2}}{32} \frac{M e_{c}^{2}}{(2 \pi)^{3}} \frac{(1-x)^{4}}{\left(P^{+}\right)^{2}\left[L_{a}^{2}\left(\Lambda_{a}^{2}\right)+\boldsymbol{p}_{T}^{2}\right]^{3}}\left\{X\left(x, \boldsymbol{p}_{T}^{2}\right)\left[\boldsymbol{p}_{T}^{2} \mathcal{I}_{1}^{\prime d i p}+\left(\frac{\boldsymbol{p}_{T}^{2}}{2 M_{a}^{2}}-\frac{1}{2}\right) \mathcal{I}_{2}^{\prime d i p}-\frac{1}{4 M_{a}^{2}} \mathcal{I}_{5}^{\prime d i p}\right]\right. \\
& \left.+Y_{1}\left(x, \boldsymbol{p}_{T}^{2}\right) \mathcal{I}_{1}^{\prime d i p}+Y_{2}\left(x, \boldsymbol{p}_{T}^{2}\right) \mathcal{I}_{3}^{\prime d i p}+Y_{3}\left(x, \boldsymbol{p}_{T}^{2}\right) \mathcal{I}_{2}^{\prime d i p}+W\left(x, \boldsymbol{p}_{T}^{2}\right)\left(\mathcal{I}_{7}^{\prime d i p}-2 \boldsymbol{p}_{T} \cdot \boldsymbol{S}_{T} \mathcal{I}_{6}^{\prime d i p}\right)\right\}, \\
h_{1}^{\perp q(a)}\left(x, \boldsymbol{p}_{T}^{2}\right)= & -\frac{g_{a}^{2}}{32} \frac{M e_{c}^{2}}{(2 \pi)^{3}} \frac{(1-x)^{4}}{\left(P^{+}\right)^{2}\left[L_{a}^{2}\left(\Lambda_{a}^{2}\right)+\boldsymbol{p}_{T}^{2}\right]^{3}}\left\{X\left(x, \boldsymbol{p}_{T}^{2}\right)\left[-\boldsymbol{p}_{T}^{2} \mathcal{I}_{1}^{\prime d i p}+\left(\frac{1}{2}-\frac{\boldsymbol{p}_{T}^{2}}{2 M_{a}^{2}}\right) \mathcal{I}_{2}^{\prime d i p}+\frac{1}{4 M_{a}^{2}} \mathcal{I}_{5}^{\prime d i p}\right]\right. \\
& +Y_{1}\left(x, \boldsymbol{p}_{T}^{2}\right) \mathcal{I}_{1}^{\prime d i p}-Y_{2}\left(x, \boldsymbol{p}_{T}^{2}\right) \mathcal{I}_{3}^{\prime d i p}-Y_{3}\left(x, \boldsymbol{p}_{T}^{2}\right) \mathcal{I}_{2}^{\prime d i p}+2 M_{a}^{2}[(m+x M)(1-x)-m x] \mathcal{I}_{1}^{\prime d i p} \\
& \left.+M_{a}^{2}\left[m\left(\kappa_{a}-1\right)-M\left(1+\kappa_{a}\right) x\right] \mathcal{I}_{2}^{\prime d i p}\right\} .
\end{aligned}
$$

- Exponential form factor

$$
\begin{aligned}
2 \operatorname{Im} J_{1}^{a}= & g_{a} \int \frac{d^{4} l}{(2 \pi)^{4}} \frac{e^{\left[(p-l)^{2}-m^{2}\right] / \Lambda_{a}^{2}}}{D_{1} D_{3}}(2 \pi i) \delta\left(D_{2}\right)(-2 \pi i) \delta\left(D_{4}\right) \\
\times & \left\{X\left(x, \boldsymbol{p}_{T}^{2}\right)\left(1+\frac{\boldsymbol{l}_{T}^{2}}{2 M_{a}^{2}}\right) \boldsymbol{l}_{T} \cdot\left(\boldsymbol{p}_{T}-\frac{1}{2} \boldsymbol{l}_{T}\right)+\right. \\
& W\left(x, \boldsymbol{p}_{T}^{2}\right)\left(\frac{\boldsymbol{l}_{T} \cdot \boldsymbol{S}_{T}}{\boldsymbol{S}_{T}^{2}}\right) \boldsymbol{S}_{T} \cdot\left[\boldsymbol{l}_{T}-2 \boldsymbol{p}_{T}\right]+ \\
& \left.\left(\frac{\boldsymbol{l}_{T} \cdot \boldsymbol{p}_{T}}{\boldsymbol{p}_{T}^{2}}\right)\left[Y_{1}\left(x, \boldsymbol{p}_{T}^{2}\right)+\boldsymbol{l}_{T} \cdot \boldsymbol{p}_{T} Y_{2}\left(x, \boldsymbol{p}_{T}^{2}\right)+\boldsymbol{l}_{T}^{2} Y_{3}\left(x, \boldsymbol{p}_{T}^{2}\right)\right]\right\} \\
= & \frac{1}{2 P^{+}}\left\{X\left(x, \boldsymbol{p}_{T}^{2}\right)\left[\boldsymbol{p}_{T}^{2} \mathcal{I}_{1}^{\text {exp }}+\left(\frac{\boldsymbol{p}_{T}^{2}}{2 M_{a}^{2}}-\frac{1}{2}\right) \mathcal{I}_{2}^{\text {exp }}-\frac{1}{4 M_{a}^{2}} \mathcal{I}_{5}^{\text {exp }}\right]+\right. \\
& Y_{1}\left(x, \boldsymbol{p}_{T}^{2}\right) \mathcal{I}_{1}^{\text {exp }}+Y_{2}\left(x, \boldsymbol{p}_{T}^{2}\right) \mathcal{I}_{3}^{\text {exp }}+Y_{3}\left(x, \boldsymbol{p}_{T}^{2}\right) \mathcal{I}_{2}^{\text {exp }}+ \\
& \left.W\left(x, \boldsymbol{p}_{T}^{2}\right)\left(\mathcal{I}_{7}^{\text {exp }}-2 \boldsymbol{p}_{T} \cdot \boldsymbol{S}_{T} \mathcal{I}_{6}^{\exp }\right)\right\} \\
2 \operatorname{Im} J_{1}^{\prime a}= & g_{a} \int \frac{d^{4} l}{(2 \pi)^{4}} \frac{e^{\left[(p-l)^{2}-m^{2}\right] / \Lambda_{a}^{2}}}{D_{1} D_{3}}(2 \pi i) \delta\left(D_{2}\right)(-2 \pi i) \delta\left(D_{4}\right)
\end{aligned}
$$




$$
\begin{aligned}
& \times\left\{-X\left(x, \boldsymbol{p}_{T}^{2}\right)\left(1+\frac{\boldsymbol{l}_{T}^{2}}{2 M_{a}^{2}}\right) \boldsymbol{l}_{T} \cdot\left(\boldsymbol{p}_{T}-\frac{1}{2} \boldsymbol{l}_{T}\right)+\right. \\
& \quad\left(\frac{\boldsymbol{l}_{T} \cdot \boldsymbol{p}_{T}}{\boldsymbol{p}_{T}^{2}}\right)\left[Y_{1}\left(x, \boldsymbol{p}_{T}^{2}\right)-\boldsymbol{l}_{T} \cdot \boldsymbol{p}_{T} Y_{2}\left(x, \boldsymbol{p}_{T}^{2}\right)-\boldsymbol{l}_{T}^{2} Y_{3}\left(x, \boldsymbol{p}_{T}^{2}\right)+\right. \\
& \left.\left.\quad+2 M_{a}^{2}[(m+x M)(1-x)-m x]+M_{a}^{2}\left[m\left(\kappa_{a}-1\right)-M\left(1+\kappa_{a}\right) x\right] \boldsymbol{l}_{T}^{2}\right]\right\} \\
& =\frac{1}{2 P^{+}}\left\{X\left(x, \boldsymbol{p}_{T}^{2}\right)\left[-\boldsymbol{p}_{T}^{2} \mathcal{I}_{1}^{\text {exp }}+\left(\frac{1}{2}-\frac{\boldsymbol{p}_{T}^{2}}{2 M_{a}^{2}}\right) \mathcal{I}_{2}^{\text {exp }}+\frac{1}{4 M_{a}^{2}} \mathcal{I}_{5}^{\text {exp }}\right]+\right. \\
& \quad Y_{1}\left(x, \boldsymbol{p}_{T}^{2}\right) \mathcal{I}_{1}^{\text {exp }}-Y_{2}\left(x, \boldsymbol{p}_{T}^{2}\right) \mathcal{I}_{3}^{\text {exp }}-Y_{3}\left(x, \boldsymbol{p}_{T}^{2}\right) \mathcal{I}_{2}^{\text {exp }}+ \\
& \left.\quad 2 M_{a}^{2}[(m+x M)(1-x)-m x] \mathcal{I}_{1}^{\text {exp }}+M_{a}^{2}\left[m\left(\kappa_{a}-1\right)-M\left(1+\kappa_{a}\right) x\right] \mathcal{I}_{2}^{\text {exp }}\right\},
\end{aligned}
$$

where the integrals $X, W, Y_{i}, i=1-3$, and $\mathcal{I}_{i}^{e x p}, i=1-7$, are listed in App. C]

The final result is, then,

$$
\begin{aligned}
f_{1 T}^{\perp q(a)}\left(x, \boldsymbol{p}_{T}^{2}\right)= & -\frac{g_{a}^{2}}{32} \frac{M e_{c}^{2}}{(2 \pi)^{3}} \frac{e^{-\left[\boldsymbol{p}_{T}^{2}+L_{a}^{2}\left(m^{2}\right)\right] /\left[(1-x) \Lambda_{a}^{2}\right]}}{\left(P^{+}\right)^{2}\left[L_{a}^{2}\left(m^{2}\right)+\boldsymbol{p}_{T}^{2}\right]}\left\{X\left(x, \boldsymbol{p}_{T}^{2}\right)\left[\boldsymbol{p}_{T}^{2} \mathcal{I}_{1}^{\text {exp }}+\left(\frac{\boldsymbol{p}_{T}^{2}}{2 M_{a}^{2}}-\frac{1}{2}\right) \mathcal{I}_{2}^{\text {exp }}-\frac{1}{4 M_{a}^{2}} \mathcal{I}_{5}^{\text {exp }}\right]\right. \\
& \left.+Y_{1}\left(x, \boldsymbol{p}_{T}^{2}\right) \mathcal{I}_{1}^{\text {exp }}+Y_{2}\left(x, \boldsymbol{p}_{T}^{2}\right) \mathcal{I}_{3}^{\text {exp }}+Y_{3}\left(x, \boldsymbol{p}_{T}^{2}\right) \mathcal{I}_{2}^{\text {exp }}+W\left(x, \boldsymbol{p}_{T}^{2}\right)\left(\mathcal{I}_{7}^{\text {exp }}-2 \boldsymbol{p}_{T} \cdot \boldsymbol{S}_{T} \mathcal{I}_{6}^{\text {exp }}\right)\right\}, \\
h_{1}^{\perp q(a)}\left(x, \boldsymbol{p}_{T}^{2}\right)= & -\frac{g_{a}^{2}}{32} \frac{M e_{c}^{2}}{(2 \pi)^{3}} \frac{e^{-\left[\boldsymbol{p}_{T}^{2}+L_{a}^{2}\left(m^{2}\right)\right] /\left[(1-x) \Lambda_{a}^{2}\right]}}{\left(P^{+}\right)^{2}\left[L_{a}^{2}\left(m^{2}\right)+\boldsymbol{p}_{T}^{2}\right]}\left\{X\left(x, \boldsymbol{p}_{T}^{2}\right)\left[-\boldsymbol{p}_{T}^{2} \mathcal{I}_{1}^{\text {exp }}+\left(\frac{1}{2}-\frac{\boldsymbol{p}_{T}^{2}}{2 M_{a}^{2}}\right) \mathcal{I}_{2}^{\text {exp }}+\frac{1}{4 M_{a}^{2}} \mathcal{I}_{5}^{\text {exp }}\right]\right. \\
& +Y_{1}\left(x, \boldsymbol{p}_{T}^{2}\right) \mathcal{I}_{1}^{\text {exp }}-Y_{2}\left(x, \boldsymbol{p}_{T}^{2}\right) \mathcal{I}_{3}^{\text {exp }}-Y_{3}\left(x, \boldsymbol{p}_{T}^{2}\right) \mathcal{I}_{2}^{\text {exp }}+2 M_{a}^{2}[(m+x M)(1-x)-m x] \mathcal{I}_{1}^{\text {exp }} \\
& \left.+M_{a}^{2}\left[m\left(\kappa_{a}-1\right)-M\left(1+\kappa_{a}\right) x\right] \mathcal{I}_{2}^{\text {exp }}\right\} .
\end{aligned}
$$

\section{Axial-vector diquark including also time-like polarization}

We have

$$
\begin{aligned}
& f_{1 T}^{\perp q(a)}\left(x, \boldsymbol{p}_{T}^{2}\right)=\frac{g_{a}\left(p^{2}\right)}{4} \frac{1}{(2 \pi)^{3}} \frac{M e_{c}^{2}}{4(1-x) P^{+}} \frac{2 \operatorname{Im} J_{1}^{a}}{p^{2}-m^{2}} \\
& h_{1}^{\perp q(a)}\left(x, \boldsymbol{p}_{T}^{2}\right)=\frac{g_{a}\left(p^{2}\right)}{4} \frac{1}{(2 \pi)^{3}} \frac{M e_{c}^{2}}{4(1-x) P^{+}} \frac{2 \operatorname{Im} J_{1}^{\prime a}}{p^{2}-m^{2}}
\end{aligned}
$$

where the $J_{1}^{a}$ and $J_{1}^{\prime a}$ integrals are defined as in Eqs. (B9) and (B10), respectively, but now the last line in Eq. (10)) is employed for the $d_{\mu \nu}(p-l-P)$ and $d_{\sigma \alpha}(P-p)$ Lorentz structures.

- Point-like coupling (to avoid divergences we assume that the $\boldsymbol{p}_{T}^{2}$ integration is extended up to a finite cutoff $\Lambda_{a}^{2}$ )

$$
\begin{aligned}
2 \operatorname{Im} J_{1}^{a} & =-4 P^{+} x\left[m\left(2 \kappa_{a}+1\right)+M\left(2 \kappa_{a} x+1\right)\right] g_{a} \mathcal{I}_{1}^{p . l .} \\
& =-g_{a} \frac{P^{+} x\left[m\left(2 \kappa_{a}+1\right)+M\left(2 \kappa_{a} x+1\right)\right]}{\pi \boldsymbol{p}_{T}^{2}} \log \left(\frac{L_{a}^{2}\left(m^{2}\right)+\boldsymbol{p}_{T}^{2}}{L_{a}^{2}\left(m^{2}\right)}\right) \\
2 \operatorname{Im} J_{1}^{\prime a} & =4 P^{+}\left[m\left[\left(2 \kappa_{a}-1\right) x+2\right]+x M\left[\left(\kappa_{a}-1\right) 2 x+3\right]\right] g_{a} \mathcal{I}_{1}^{p . l .} \\
& =g_{a} \frac{P^{+}\left[m\left[\left(2 \kappa_{a}-1\right) x+2\right]+x M\left[\left(\kappa_{a}-1\right) 2 x+3\right]\right]}{\pi \boldsymbol{p}_{T}^{2}} \log \left(\frac{L_{a}^{2}\left(m^{2}\right)+\boldsymbol{p}_{T}^{2}}{L_{a}^{2}\left(m^{2}\right)}\right),
\end{aligned}
$$

where $\mathcal{I}_{1}^{p . l .}$ is the same integral as in Eq. ( (B4) but with the substitution $L_{s}\left(m^{2}\right) \leftrightarrow L_{a}\left(m^{2}\right)$. 
Using again Eq. (8), the final result is

$$
\begin{aligned}
& f_{1 T}^{\perp q(a)}\left(x, \boldsymbol{p}_{T}^{2}\right)=\frac{g_{a}^{2}}{8} \frac{M e_{c}^{2}}{(2 \pi)^{4}} \frac{x\left[m\left(2 \kappa_{a}+1\right)+M\left(2 \kappa_{a} x+1\right)\right]}{\boldsymbol{p}_{T}^{2}\left[L_{a}^{2}\left(m^{2}\right)+\boldsymbol{p}_{T}^{2}\right]} \log \left(\frac{L_{a}^{2}\left(m^{2}\right)+\boldsymbol{p}_{T}^{2}}{L_{a}^{2}\left(m^{2}\right)}\right) \\
& h_{1}^{\perp q(a)}\left(x, \boldsymbol{p}_{T}^{2}\right)=-\frac{g_{a}^{2}}{8} \frac{M e_{c}^{2}}{(2 \pi)^{4}} \frac{m\left[\left(2 \kappa_{a}-1\right) x+2\right]+x M\left[\left(\kappa_{a}-1\right) 2 x+3\right]}{\boldsymbol{p}_{T}^{2}\left[L_{a}^{2}\left(m^{2}\right)+\boldsymbol{p}_{T}^{2}\right]} \log \left(\frac{L_{a}^{2}\left(m^{2}\right)+\boldsymbol{p}_{T}^{2}}{L_{a}^{2}\left(m^{2}\right)}\right) .
\end{aligned}
$$

- Dipolar form factor

$$
\begin{aligned}
2 \operatorname{Im} J_{1}^{a} & =4 P^{+} x(1-x)\left[m\left(2 \kappa_{a}+1\right)+M\left(2 \kappa_{a} x+1\right)\right] g_{a} \mathcal{I}_{1}^{d i p} \\
& =g_{a} \frac{P^{+} x(1-x)\left[m\left(2 \kappa_{a}+1\right)+M\left(2 \kappa_{a} x+1\right)\right]}{\pi L_{a}^{2}\left(\Lambda_{a}^{2}\right)\left[L_{a}^{2}\left(\Lambda_{a}^{2}\right)+\boldsymbol{p}_{T}^{2}\right]} \\
2 \operatorname{Im} J_{1}^{\prime a} & =-4 P^{+}(1-x)\left[m\left[\left(2 \kappa_{a}-1\right) x+2\right]+x M\left[\left(\kappa_{a}-1\right) 2 x+3\right]\right] g_{a} \mathcal{I}_{1}^{d i p} \\
& =-g_{a} \frac{P^{+}(1-x)\left[m\left[\left(2 \kappa_{a}-1\right) x+2\right]+x M\left[\left(\kappa_{a}-1\right) 2 x+3\right]\right]}{\pi L_{a}^{2}\left(\Lambda_{a}^{2}\right)\left[L_{a}^{2}\left(\Lambda_{a}^{2}\right)+\boldsymbol{p}_{T}^{2}\right]} .
\end{aligned}
$$

The final result is

$$
\begin{aligned}
& f_{1 T}^{\perp q(a)}\left(x, \boldsymbol{p}_{T}^{2}\right)=\frac{g_{a}^{2}}{8} \frac{M e_{c}^{2}}{(2 \pi)^{4}} \frac{x(1-x)^{2}\left[m\left(2 \kappa_{a}+1\right)+M\left(2 \kappa_{a} x+1\right)\right]}{L_{a}^{2}\left(\Lambda_{a}^{2}\right)\left[L_{a}^{2}\left(\Lambda_{a}^{2}\right)+\boldsymbol{p}_{T}^{2}\right]^{3}}, \\
& h_{1}^{\perp q(a)}\left(x, \boldsymbol{p}_{T}^{2}\right)=-\frac{g_{a}^{2}}{8} \frac{M e_{c}^{2}}{(2 \pi)^{4}} \frac{(1-x)^{2}\left[m\left[\left(2 \kappa_{a}-1\right) x+2\right]+x M\left[\left(\kappa_{a}-1\right) 2 x+3\right]\right]}{L_{a}^{2}\left(\Lambda_{a}^{2}\right)\left[L_{a}^{2}\left(\Lambda_{a}^{2}\right)+\boldsymbol{p}_{T}^{2}\right]^{3}} .
\end{aligned}
$$

We find a discrepancy between these results and those of Eqs. (18) and (24) in Ref. [46], probably due to errors in that calculation.

- Exponential form factor

$$
\begin{aligned}
2 \operatorname{Im} J_{1}^{a} & =-4 P^{+} x\left[m\left(2 \kappa_{a}+1\right)+M\left(2 \kappa_{a} x+1\right)\right] g_{a} \mathcal{I}_{1}^{\exp } \\
& =-g_{a} \frac{P^{+} x\left[m\left(2 \kappa_{a}+1\right)+M\left(2 \kappa_{a} x+1\right)\right]}{\pi \boldsymbol{p}_{T}^{2}}\left[\Gamma\left(0, \frac{L_{a}^{2}\left(m^{2}\right)}{(1-x) \Lambda_{a}^{2}}\right)-\Gamma\left(0, \frac{L_{a}^{2}\left(m^{2}\right)+\boldsymbol{p}_{T}^{2}}{(1-x) \Lambda_{a}^{2}}\right)\right], \\
2 \operatorname{Im} J_{1}^{\prime a} & =4 P^{+}\left[m\left[\left(2 \kappa_{a}-1\right) x+2\right]+x M\left[\left(\kappa_{a}-1\right) 2 x+3\right]\right] g_{a} \mathcal{I}_{1}^{\text {exp }} \\
& =g_{a} \frac{P^{+}\left[m\left[\left(2 \kappa_{a}-1\right) x+2\right]+x M\left[\left(\kappa_{a}-1\right) 2 x+3\right]\right]}{\pi \boldsymbol{p}_{T}^{2}}\left[\Gamma\left(0, \frac{L_{a}^{2}\left(m^{2}\right)}{(1-x) \Lambda_{a}^{2}}\right)-\Gamma\left(0, \frac{L_{a}^{2}\left(m^{2}\right)+\boldsymbol{p}_{T}^{2}}{(1-x) \Lambda_{a}^{2}}\right)\right] .
\end{aligned}
$$

The final result is, then,

$$
\begin{gathered}
f_{1 T}^{\perp q(a)}\left(x, \boldsymbol{p}_{T}^{2}\right)=\frac{g_{a}^{2}}{8} \frac{M e_{c}^{2}}{(2 \pi)^{4}} \frac{x\left[m\left(2 \kappa_{a}+1\right)+M\left(2 \kappa_{a} x+1\right)\right]}{\boldsymbol{p}_{T}^{2}\left[L_{a}^{2}\left(m^{2}\right)+\boldsymbol{p}_{T}^{2}\right]} e^{-\left[\boldsymbol{p}_{T}^{2}+L_{a}^{2}\left(m^{2}\right)\right] /\left[(1-x) \Lambda_{a}^{2}\right]} \\
\times\left[\Gamma\left(0, \frac{L_{a}^{2}\left(m^{2}\right)}{(1-x) \Lambda_{a}^{2}}\right)-\Gamma\left(0, \frac{L_{a}^{2}\left(m^{2}\right)+\boldsymbol{p}_{T}^{2}}{(1-x) \Lambda_{a}^{2}}\right)\right], \\
h_{1}^{\perp q(a)}\left(x, \boldsymbol{p}_{T}^{2}\right)=-\frac{g_{a}^{2}}{8} \frac{M e_{c}^{2}}{(2 \pi)^{4}} \frac{m\left[\left(2 \kappa_{a}-1\right) x+2\right]+x M\left[\left(\kappa_{a}-1\right) 2 x+3\right]}{\boldsymbol{p}_{T}^{2}\left[L_{a}^{2}\left(m^{2}\right)+\boldsymbol{p}_{T}^{2}\right]} e^{-\left[\boldsymbol{p}_{T}^{2}+L_{a}^{2}\left(m^{2}\right)\right] /\left[(1-x) \Lambda_{a}^{2}\right]} \\
\times\left[\Gamma\left(0, \frac{L_{a}^{2}\left(m^{2}\right)}{(1-x) \Lambda_{a}^{2}}\right)-\Gamma\left(0, \frac{L_{a}^{2}\left(m^{2}\right)+\boldsymbol{p}_{T}^{2}}{(1-x) \Lambda_{a}^{2}}\right)\right] .
\end{gathered}
$$

\section{APPENDIX C: USEFUL INTEGRALS}

In this appendix, we calculate the relevant integrals that repeatedly show up in the expressions of T-odd parton densities for all choices of nucleon-quark-diquark form factors, when vector diquark propagators are represented in the first and last forms of Eq. (10). 
We will systematically use the substitutions $\boldsymbol{l}_{T}^{\prime}=\boldsymbol{l}_{T}-\boldsymbol{p}_{T}$ and $y=\boldsymbol{l}_{T}^{\prime 2}+L_{X}^{2}\left(m^{2}\right)$, or $y=\boldsymbol{l}_{T}^{\prime 2}+L_{X}^{2}\left(\Lambda_{X}^{2}\right)$ for the dipolar form factor, where $L_{X}$ is defined in Eq. (8) for $X=s, a$ scalar and axial-vector diquarks, respectively. We will also encounter the following angular integrals, where $\theta$ is defined as the angle between $\boldsymbol{l}_{T}^{\prime}$ and $\boldsymbol{p}_{T}$, and $\phi, \phi_{S}$, are the azimuthal angles of $\boldsymbol{p}_{T}$ and $\boldsymbol{S}_{T}$ with respect to the scattering plane:

$$
\begin{aligned}
& \int_{0}^{2 \pi} d \theta \frac{\left|\boldsymbol{l}_{T}^{\prime}\right|\left|\boldsymbol{p}_{T}\right| \cos \theta+\boldsymbol{p}_{T}^{2}}{\boldsymbol{l}_{T}^{\prime 2}+\boldsymbol{p}_{T}^{2}+2\left|\boldsymbol{l}_{T}^{\prime}\right|\left|\boldsymbol{p}_{T}\right| \cos \theta}=\pi\left(1-\operatorname{sgn}\left(\left|\boldsymbol{l}_{T}^{\prime}\right|-\left|\boldsymbol{p}_{T}\right|\right)\right) \\
& \int_{0}^{2 \pi} d \theta \frac{\left[\left|\boldsymbol{l}_{T}^{\prime}\right|\left|\boldsymbol{p}_{T}\right| \cos \theta+\boldsymbol{p}_{T}^{2}\right]^{2}}{\boldsymbol{l}_{T}^{\prime 2}+\boldsymbol{p}_{T}^{2}+2\left|\boldsymbol{l}_{T}^{\prime}\right|\left|\boldsymbol{p}_{T}\right| \cos \theta}=\frac{\pi}{2}\left(-\boldsymbol{l}_{T}^{\prime 2}+3 \boldsymbol{p}_{T}^{2}+\left|\boldsymbol{l}_{T}^{\prime 2}-\boldsymbol{p}_{T}^{2}\right|\right) \\
& \int_{0}^{2 \pi} d \theta \frac{\left|\boldsymbol{l}_{T}^{\prime}\right|\left|\boldsymbol{S}_{T}\right| \cos \left[\theta+\left(\phi-\phi_{S}\right)\right]+\left|\boldsymbol{p}_{T}\right|\left|\boldsymbol{S}_{T}\right| \cos \left(\phi-\phi_{S}\right)}{\boldsymbol{l}_{T}^{\prime 2}+\boldsymbol{p}_{T}^{2}+2\left|\boldsymbol{l}_{T}^{\prime}\right|\left|\boldsymbol{p}_{T}\right| \cos \theta}=\pi \frac{\left|\boldsymbol{S}_{T}\right|}{\left|\boldsymbol{p}_{T}\right|} \cos \left(\phi-\phi_{S}\right)\left(\operatorname{sgn}\left(\left|\boldsymbol{p}_{T}\right|-\left|\boldsymbol{l}_{T}^{\prime}\right|\right)+1\right) \\
& \int_{0}^{2 \pi} d \theta \frac{\left(\left|\boldsymbol{l}_{T}^{\prime}\right|\left|\boldsymbol{S}_{T}\right| \cos \left[\theta+\left(\phi-\phi_{S}\right)\right]+\left|\boldsymbol{p}_{T}\right|\left|\boldsymbol{S}_{T}\right| \cos \left(\phi-\phi_{S}\right)\right)^{2}}{\boldsymbol{l}_{T}^{\prime 2}+\boldsymbol{p}_{T}^{2}+2\left|\boldsymbol{l}_{T}^{\prime}\right|\left|\boldsymbol{p}_{T}\right| \cos \theta}=\pi \frac{\boldsymbol{S}_{T}^{2}}{2 \boldsymbol{p}_{T}^{2}}\left[\left(\boldsymbol{p}_{T}^{2}-\boldsymbol{l}_{T}^{\prime 2}+\left|\boldsymbol{p}_{T}^{2}-\boldsymbol{l}_{T}^{\prime 2}\right|\right) \cos 2\left(\phi-\phi_{S}\right)\right. \\
& \left.+2 \boldsymbol{p}_{T}^{2}\right] .
\end{aligned}
$$

- Point-like coupling

$$
\begin{aligned}
\mathcal{I}_{1}^{p . l .} & =\int \frac{d \boldsymbol{l}_{T}^{\prime}}{(2 \pi)^{2}} \frac{\left(\boldsymbol{l}_{T}^{\prime}+\boldsymbol{p}_{T}\right) \cdot \boldsymbol{p}_{T}}{\boldsymbol{p}_{T}^{2}} \frac{1}{\left(\boldsymbol{l}_{T}^{\prime}+\boldsymbol{p}_{T}\right)^{2}\left[\boldsymbol{l}_{T}^{\prime 2}+L_{X}^{2}\left(m^{2}\right)\right]} \\
& =\frac{1}{(2 \pi)^{2} \boldsymbol{p}_{T}^{2}} \int_{0}^{\infty} d\left|\boldsymbol{l}_{T}^{\prime}\right|\left|\boldsymbol{l}_{T}^{\prime}\right| \frac{1}{\boldsymbol{l}_{T}^{\prime 2}+L_{X}^{2}\left(m^{2}\right)} \int_{0}^{2 \pi} d \theta \frac{\left|\boldsymbol{l}_{T}^{\prime}\right|\left|\boldsymbol{p}_{T}\right| \cos \theta+\boldsymbol{p}_{T}^{2}}{\boldsymbol{l}_{T}^{\prime 2}+\boldsymbol{p}_{T}^{2}+2\left|\boldsymbol{l}_{T}^{\prime}\right|\left|\boldsymbol{p}_{T}\right| \cos \theta} \\
& =\frac{1}{(2 \pi) \boldsymbol{p}_{T}^{2}} \int_{0}^{\left|\boldsymbol{p}_{T}\right|} d\left|\boldsymbol{l}_{T}^{\prime}\right|\left|\boldsymbol{l}_{T}^{\prime}\right| \frac{1}{\boldsymbol{l}_{T}^{\prime 2}+L_{X}^{2}\left(m^{2}\right)} \\
& =\frac{1}{4 \pi \boldsymbol{p}_{T}^{2}} \int_{L_{X}^{2}\left(m^{2}\right)}^{L_{X}^{2}\left(m^{2}\right)+\boldsymbol{p}_{T}^{2}} \frac{d y}{y}=\frac{1}{4 \pi \boldsymbol{p}_{T}^{2}} \log \left(\frac{L_{X}^{2}\left(m^{2}\right)+\boldsymbol{p}_{T}^{2}}{L_{X}^{2}\left(m^{2}\right)}\right)
\end{aligned}
$$

- Dipolar form factor

$$
\begin{aligned}
\mathcal{I}_{1}^{d i p} & =-\int \frac{d \boldsymbol{l}_{T}^{\prime}}{(2 \pi)^{2}} \frac{\left(\boldsymbol{l}_{T}^{\prime}+\boldsymbol{p}_{T}\right) \cdot \boldsymbol{p}_{T}}{\boldsymbol{p}_{T}^{2}} \frac{(1-x)}{\left(\boldsymbol{l}_{T}^{\prime}+\boldsymbol{p}_{T}\right)^{2}\left[\boldsymbol{l}_{T}^{\prime 2}+L_{X}^{2}\left(\Lambda_{X}^{2}\right)\right]^{2}} \\
& =-\frac{(1-x)}{4 \pi \boldsymbol{p}_{T}^{2}} \int_{L_{X}^{2}\left(\Lambda_{X}^{2}\right)}^{L_{X}^{2}\left(\Lambda_{X}^{2}\right)+\boldsymbol{p}_{T}^{2}} \frac{d y}{y^{2}}=-\frac{(1-x)}{4 \pi L_{X}^{2}\left(\Lambda_{X}^{2}\right)\left[L_{X}^{2}\left(\Lambda_{X}^{2}\right)+\boldsymbol{p}_{T}^{2}\right]}
\end{aligned}
$$

- Exponential form factor

$$
\begin{aligned}
\mathcal{I}_{1}^{\text {exp }} & =\int \frac{d \boldsymbol{l}_{T}^{\prime}}{(2 \pi)^{2}} \frac{\left(\boldsymbol{l}_{T}^{\prime}+\boldsymbol{p}_{T}\right) \cdot \boldsymbol{p}_{T}}{\boldsymbol{p}_{T}^{2}} \frac{e^{-\left[\boldsymbol{l}_{T}^{\prime 2}+L_{X}^{2}\left(m^{2}\right)\right] /\left[(1-x) \Lambda_{X}^{2}\right]}}{\left(\boldsymbol{l}_{T}^{\prime}+\boldsymbol{p}_{T}\right)^{2}\left[\boldsymbol{l}_{T}^{\prime 2}+L_{X}^{2}\left(m^{2}\right)\right]} \\
& =\frac{1}{(2 \pi) \boldsymbol{p}_{T}^{2}} \int_{0}^{\left|\boldsymbol{p}_{T}\right|} d\left|\boldsymbol{l}_{T}^{\prime}\right|\left|\boldsymbol{l}_{T}^{\prime}\right| \frac{e^{-\left[\boldsymbol{l}_{T}^{\prime 2}+L_{X}^{2}\left(m^{2}\right)\right] /\left[(1-x) \Lambda_{X}^{2}\right]}}{\boldsymbol{l}_{T}^{\prime 2}+L_{X}^{2}\left(m^{2}\right)} \\
& =\frac{1}{(4 \pi) \boldsymbol{p}_{T}^{2}} \int_{L_{X}^{2}\left(m^{2}\right)}^{L_{X}^{2}\left(m^{2}\right)+\boldsymbol{p}_{T}^{2}} \frac{d y}{y} e^{-y /\left[(1-x) \Lambda_{X}^{2}\right]} \\
& =\frac{1}{4 \pi \boldsymbol{p}_{T}^{2}}\left[\Gamma\left(0, \frac{L_{X}^{2}\left(m^{2}\right)}{(1-x) \Lambda_{X}^{2}}\right)-\Gamma\left(0, \frac{L_{X}^{2}\left(m^{2}\right)+\boldsymbol{p}_{T}^{2}}{(1-x) \Lambda_{X}^{2}}\right)\right] .
\end{aligned}
$$

Next, we list the coefficients and calculate the relevant integrals that are needed to construct T-odd parton densities for all choices of nucleon-quark-diquark form factors, when vector diquarks are represented in the second form in Eq. (10). 


$$
\begin{aligned}
& X=-\frac{16(m+M)\left(P^{+}\right)^{2} \kappa_{a}}{M_{a}^{2}}, \\
& W=-\frac{8(m+M)\left(P^{+}\right)^{2}\left(\kappa_{a}-1\right)(1-x)}{M_{a}^{2}}, \\
& Y_{1}=-\frac{8\left(P^{+}\right)^{2}}{M_{a}^{2}}\left[\kappa _ { a } \left(2 m^{3}-3 x m^{3}+M x^{2} m^{2}+2 M m^{2}-3 M x m^{2}-M^{2} x m+2 M_{a}^{2} x m+M^{3} x^{2}-M^{3} x\right.\right. \\
& \left.-\left[\boldsymbol{p}_{T}^{2}+L_{a}^{2}\left(m^{2}\right)\right](M+m)\right)+\left(1+\kappa_{a}\right) M_{a}^{2} x[M(1+x)+m]+\left[\boldsymbol{p}_{T}^{2}+L_{a}^{2}\left(m^{2}\right)\right] m\left(\kappa_{a}-1\right)(x-1) \\
& \left.+m\left(\kappa_{a}-1\right) x^{2}\left(M_{a}^{2}+m^{2}\right)+m^{3}(2 x-1)+M^{2} m\left(1-2 x+\left(1+\kappa_{a}\right) x^{2}\right)\right] \text {, } \\
& Y_{2}=-\frac{8 m\left(P^{+}\right)^{2}\left(\kappa_{a}-1\right)(1-x)}{M_{a}^{2}}, \\
& Y_{3}=\frac{4\left(P^{+}\right)^{2}}{M_{a}^{4}}\left[\kappa _ { a } \left(-m^{3}+x m^{3}-M x^{2} m^{2}-2 M m^{2}+3 M x m^{2}-M^{2} m-2 M^{2} x^{2} m+3 M^{2} x m-2 M_{a}^{2} x m\right.\right. \\
& \left.\left.-M^{3} x^{2}+\left[\boldsymbol{p}_{T}^{2}+L_{a}^{2}\left(m^{2}\right)\right](m+M)+M^{3} x\right)+M_{a}^{2} m\left(\kappa_{a}-1\right)-M M_{a}^{2}\left(1+\kappa_{a}\right) x\right], \\
& \mathcal{I}_{1}^{\prime d i p}=\int \frac{d \boldsymbol{l}_{T}^{\prime}}{(2 \pi)^{2}} \frac{\left(\boldsymbol{l}_{T}^{\prime}+\boldsymbol{p}_{T}\right) \cdot \boldsymbol{p}_{T}}{\boldsymbol{p}_{T}^{2}} \frac{1}{\left(\boldsymbol{l}_{T}^{\prime}+\boldsymbol{p}_{T}\right)^{2}\left[\boldsymbol{l}_{T}^{\prime 2}+L_{a}^{2}\left(\Lambda_{a}^{2}\right)\right]^{3}} \\
& =\int_{0}^{\infty} \frac{d\left|\boldsymbol{l}_{T}^{\prime}\right|\left|\boldsymbol{l}_{T}^{\prime}\right|}{(2 \pi)^{2} \boldsymbol{p}_{T}^{2}} \frac{1}{\left[\boldsymbol{l}_{T}^{\prime 2}+L_{a}^{2}\left(\Lambda_{a}^{2}\right)\right]^{3}} \int_{0}^{2 \pi} d \theta \frac{\left|\boldsymbol{l}_{T}^{\prime}\right|\left|\boldsymbol{p}_{T}\right| \cos \theta+\boldsymbol{p}_{T}^{2}}{\boldsymbol{l}_{T}^{\prime 2}+\boldsymbol{p}_{T}^{2}+2\left|\boldsymbol{l}_{T}^{\prime}\right|\left|\boldsymbol{p}_{T}\right| \cos \theta} \\
& =\int_{0}^{\left|\boldsymbol{p}_{T}\right|} \frac{d\left|\boldsymbol{l}_{T}^{\prime}\right|\left|\boldsymbol{l}_{T}^{\prime}\right|}{(2 \pi) \boldsymbol{p}_{T}^{2}} \frac{1}{\left[\boldsymbol{l}_{T}^{\prime 2}+L_{a}^{2}\left(\Lambda_{a}^{2}\right)\right]^{3}}=\frac{1}{4 \pi \boldsymbol{p}_{T}^{2}} \int_{L_{a}^{2}\left(\Lambda_{a}^{2}\right)}^{L_{a}^{2}\left(\Lambda_{a}^{2}\right)+\boldsymbol{p}_{T}^{2}} \frac{d y}{y^{3}} \\
& =\frac{1}{8 \pi \boldsymbol{p}_{T}^{2}}\left[\frac{1}{L_{a}^{4}\left(\Lambda_{a}^{2}\right)}-\frac{1}{\left[L_{a}^{2}\left(\Lambda_{a}^{2}\right)+\boldsymbol{p}_{T}^{2}\right]^{2}}\right] \\
& =\frac{2 L_{a}^{2}\left(\Lambda_{a}^{2}\right)+\boldsymbol{p}_{T}^{2}}{8 \pi L_{a}^{4}\left(\Lambda_{a}^{2}\right)\left[L_{a}^{2}\left(\Lambda_{a}^{2}\right)+\boldsymbol{p}_{T}^{2}\right]^{2}}, \\
& \mathcal{I}_{2}^{\prime d i p}=\int \frac{d \boldsymbol{l}_{T}^{\prime}}{(2 \pi)^{2}} \frac{\left(\boldsymbol{l}_{T}^{\prime}+\boldsymbol{p}_{T}\right)^{2}}{\left(\boldsymbol{l}_{T}^{\prime}+\boldsymbol{p}_{T}\right)^{2}\left[\boldsymbol{l}_{T}^{\prime 2}+L_{a}^{2}\left(\Lambda_{a}^{2}\right)\right]^{3}} \\
& =\int_{0}^{\infty} \frac{d\left|\boldsymbol{l}_{T}^{\prime}\right|\left|\boldsymbol{l}_{T}^{\prime}\right|}{(2 \pi)^{2}} \frac{1}{\left[\boldsymbol{l}_{T}^{\prime 2}+L_{a}^{2}\left(\Lambda_{a}^{2}\right)\right]^{3}} \int_{0}^{2 \pi} d \theta=\frac{1}{4 \pi} \int_{L_{a}^{2}\left(\Lambda_{a}^{2}\right)}^{\infty} \frac{d y}{y^{3}} \\
& =\frac{1}{8 \pi L_{a}^{4}\left(\Lambda_{a}^{2}\right)} \text {, } \\
& \mathcal{I}_{3}^{\prime d i p}=\int \frac{d^{2} \boldsymbol{l}_{T}^{\prime}}{(2 \pi)^{2}} \frac{\left[\left(\boldsymbol{l}_{T}^{\prime}+\boldsymbol{p}_{T}\right) \cdot \boldsymbol{p}_{T}\right]^{2}}{\boldsymbol{p}_{T}^{2}} \frac{1}{\left(\boldsymbol{l}_{T}^{\prime}+\boldsymbol{p}_{T}\right)^{2}\left[\boldsymbol{l}_{T}^{\prime 2}+L_{a}^{2}\left(\Lambda_{a}^{2}\right)\right]^{3}} \\
& =\int_{0}^{\infty} \frac{d\left|\boldsymbol{l}_{T}^{\prime}\right|\left|\boldsymbol{l}_{T}^{\prime}\right|}{(2 \pi)^{2} \boldsymbol{p}_{T}^{2}} \frac{1}{\left[\boldsymbol{l}_{T}^{\prime 2}+L_{a}^{2}\left(\Lambda_{a}^{2}\right)\right]^{3}} \int_{0}^{2 \pi} d \theta \frac{\left[\left|\boldsymbol{l}_{T}^{\prime}\right|\left|\boldsymbol{p}_{T}\right| \cos \theta+\boldsymbol{p}_{T}^{2}\right]^{2}}{\boldsymbol{l}_{T}^{\prime 2}+\boldsymbol{p}_{T}^{2}+2\left|\boldsymbol{l}_{T}^{\prime}\right|\left|\boldsymbol{p}_{T}\right| \cos \theta} \\
& =\frac{1}{4 \pi \boldsymbol{p}_{T}^{2}}\left\{\int_{0}^{\left|\boldsymbol{p}_{T}\right|} \frac{d\left|\boldsymbol{l}_{T}^{\prime}\right|\left|\boldsymbol{l}_{T}^{\prime}\right|}{\left[\boldsymbol{l}_{T}^{2}+L_{a}^{2}\left(\Lambda_{a}^{2}\right)\right]^{3}}\left(2 \boldsymbol{p}_{T}^{2}-\boldsymbol{l}_{T}^{\prime 2}\right)+\boldsymbol{p}_{T}^{2} \int_{\left|\boldsymbol{p}_{T}\right|}^{\infty} \frac{d\left|\boldsymbol{l}_{T}^{\prime}\right|\left|\boldsymbol{l}_{T}^{\prime}\right|}{\left[\boldsymbol{l}_{T}^{\prime 2}+L_{a}^{2}\left(\Lambda_{a}^{2}\right)\right]^{3}}\right\}
\end{aligned}
$$




$$
\begin{aligned}
& =\frac{1}{8 \pi \boldsymbol{p}_{T}^{2}}\left\{\int_{L_{a}^{2}\left(\Lambda_{a}^{2}\right)}^{L_{a}^{2}\left(\Lambda_{a}^{2}\right)+\boldsymbol{p}_{T}^{2}} d y \frac{2 \boldsymbol{p}_{T}^{2}+L_{a}^{2}\left(\Lambda_{a}^{2}\right)-y}{y^{3}}+\boldsymbol{p}_{T}^{2} \int_{L_{a}^{2}\left(\Lambda_{a}^{2}\right)+\boldsymbol{p}_{T}^{2}}^{\infty} \frac{d y}{y^{3}}\right\} \\
& =\frac{1}{8 \pi \boldsymbol{p}_{T}^{2}}\left\{\left(\frac{2 \boldsymbol{p}_{T}^{6}+3 L_{a}^{2}\left(\Lambda_{a}^{2}\right) \boldsymbol{p}_{T}^{4}}{2 L_{a}^{2}\left(\Lambda_{a}^{2}\right)\left[L_{a}^{2}\left(\Lambda_{a}^{2}\right)+\boldsymbol{p}_{T}^{2}\right]^{2}}\right)+\boldsymbol{p}_{T}^{2}\left(\frac{1}{2\left[L_{a}^{2}\left(\Lambda_{a}^{2}\right)+\boldsymbol{p}_{T}^{2}\right]^{2}}\right)\right\} \\
& =\frac{L_{a}^{2}\left(\Lambda_{a}^{2}\right)+2 \boldsymbol{p}_{T}^{2}}{16 \pi L_{a}^{4}\left(\Lambda_{a}^{2}\right)\left[L_{a}^{2}\left(\Lambda_{a}^{2}\right)+\boldsymbol{p}_{T}^{2}\right]}, \\
& \mathcal{I}_{4}^{\prime d i p}=\int \frac{d \boldsymbol{l}_{T}^{\prime}}{(2 \pi)^{2}} \frac{\left(\boldsymbol{l}_{T}^{\prime}+\boldsymbol{p}_{T}\right) \cdot \boldsymbol{p}_{T}}{\boldsymbol{p}_{T}^{2}} \frac{\left(\boldsymbol{l}_{T}^{\prime}+\boldsymbol{p}_{T}\right)^{2}}{\left(\boldsymbol{l}_{T}^{\prime}+\boldsymbol{p}_{T}\right)^{2}\left[\boldsymbol{l}_{T}^{\prime 2}+L_{a}^{2}\left(\Lambda_{a}^{2}\right)\right]^{3}} \\
& =\int_{0}^{\infty} \frac{d\left|\boldsymbol{l}_{T}^{\prime}\right|\left|\boldsymbol{l}_{T}^{\prime}\right|}{(2 \pi)^{2} \boldsymbol{p}_{T}^{2}} \frac{1}{\left[\boldsymbol{l}_{T}^{\prime 2}+L_{a}^{2}\left(\Lambda_{a}^{2}\right)\right]^{3}} \int_{0}^{2 \pi} d \theta\left(\left|\boldsymbol{l}_{T}^{\prime}\right|\left|\boldsymbol{p}_{T}\right| \cos \theta+\boldsymbol{p}_{T}^{2}\right) \\
& =\int_{0}^{\infty} \frac{d\left|\boldsymbol{l}_{T}^{\prime}\right|\left|\boldsymbol{l}_{T}^{\prime}\right|}{(2 \pi)} \frac{1}{\left[\boldsymbol{l}_{T}^{\prime 2}+L_{a}^{2}\left(\Lambda_{a}^{2}\right)\right]^{3}}=\frac{1}{4 \pi} \int_{L_{a}^{2}\left(\Lambda_{a}^{2}\right)}^{\infty} \frac{d y}{y^{3}} \\
& =\frac{1}{8 \pi L_{a}^{4}\left(\Lambda_{a}^{2}\right)} \equiv \mathcal{I}_{2}^{\prime d i p}, \\
& \mathcal{I}_{5}^{\prime d i p}=\int \frac{d \boldsymbol{l}_{T}^{\prime}}{(2 \pi)^{2}} \frac{\left(\boldsymbol{l}_{T}^{\prime}+\boldsymbol{p}_{T}\right)^{4}}{\left(\boldsymbol{l}_{T}^{\prime}+\boldsymbol{p}_{T}\right)^{2}\left[\boldsymbol{l}_{T}^{\prime 2}+L_{a}^{2}\left(\Lambda_{a}^{2}\right)\right]^{3}} \\
& =\int_{0}^{\infty} \frac{d\left|\boldsymbol{l}_{T}^{\prime}\right|\left|\boldsymbol{l}_{T}^{\prime}\right|}{(2 \pi)^{2}} \frac{1}{\left[\boldsymbol{l}_{T}^{\prime 2}+L_{a}^{2}\left(\Lambda_{a}^{2}\right)\right]^{3}} \int_{0}^{2 \pi} d \theta\left(\boldsymbol{l}_{T}^{\prime 2}+\boldsymbol{p}_{T}^{2}+2\left|\boldsymbol{l}_{T}^{\prime}\right|\left|\boldsymbol{p}_{T}\right| \cos \theta\right) \\
& =\int_{0}^{\infty} \frac{d\left|\boldsymbol{l}_{T}^{\prime}\right|\left|\boldsymbol{l}_{T}^{\prime}\right|}{(2 \pi)} \frac{\left(\boldsymbol{l}_{T}^{\prime 2}+\boldsymbol{p}_{T}^{2}\right)}{\left[\boldsymbol{l}_{T}^{\prime 2}+L_{a}^{2}\left(\Lambda_{a}^{2}\right)\right]^{3}}=\frac{1}{4 \pi} \int_{L_{a}^{2}\left(\Lambda_{a}^{2}\right)}^{\infty} d y \frac{y-L_{a}^{2}\left(\Lambda_{a}^{2}\right)+\boldsymbol{p}_{T}^{2}}{y^{3}} \\
& =\frac{L_{a}^{2}\left(\Lambda_{a}^{2}\right)+\boldsymbol{p}_{T}^{2}}{8 \pi L_{a}^{4}\left(\Lambda_{a}^{2}\right)} \\
& \mathcal{I}_{6}^{\prime d i p}=\int \frac{d \boldsymbol{l}_{T}^{\prime}}{(2 \pi)^{2}} \frac{\left(\boldsymbol{l}_{T}^{\prime}+\boldsymbol{p}_{T}\right) \cdot \boldsymbol{S}_{T}}{\boldsymbol{S}_{T}^{2}} \frac{1}{\left(\boldsymbol{l}_{T}^{\prime}+\boldsymbol{p}_{T}\right)^{2}\left[\boldsymbol{l}_{T}^{\prime 2}+L_{a}^{2}\left(\Lambda_{a}^{2}\right)\right]^{3}} \\
& =\int_{0}^{\infty} \frac{d\left|\boldsymbol{l}_{T}^{\prime}\right|\left|\boldsymbol{l}_{T}^{\prime}\right|}{(2 \pi)^{2} \boldsymbol{p}_{T}^{2}} \frac{1}{\left[\boldsymbol{l}_{T}^{\prime 2}+L_{a}^{2}\left(\Lambda_{a}^{2}\right)\right]^{3}} \int_{0}^{2 \pi} d \theta \frac{\left|\boldsymbol{l}_{T}^{\prime}\right|\left|\boldsymbol{S}_{T}\right| \cos \left[\theta+\left(\phi-\phi_{S}\right)\right]+\left|\boldsymbol{p}_{T}\right|\left|\boldsymbol{S}_{T}\right| \cos \left(\phi-\phi_{S}\right)}{\boldsymbol{l}_{T}^{\prime 2}+\boldsymbol{p}_{T}^{2}+2\left|\boldsymbol{l}_{T}^{\prime}\right|\left|\boldsymbol{p}_{T}\right| \cos \theta} \\
& =\frac{1}{4 \pi \boldsymbol{S}_{T}^{2}} \frac{\left|\boldsymbol{S}_{T}\right|}{\left|\boldsymbol{p}_{T}\right|} \cos \left(\phi-\phi_{S}\right) \int_{L_{a}^{2}\left(\Lambda_{a}^{2}\right)}^{L_{a}^{2}\left(\Lambda_{a}^{2}\right)+\boldsymbol{p}_{T}^{2}} \frac{d y}{y^{3}}=\frac{1}{8 \pi \boldsymbol{S}_{T}^{2}} \frac{\left|\boldsymbol{S}_{T}\right|}{\left|\boldsymbol{p}_{T}\right|}\left[\frac{1}{L_{a}^{4}\left(\Lambda_{a}^{2}\right)}-\frac{1}{\left[L_{a}^{2}\left(\Lambda_{a}^{2}\right)+\boldsymbol{p}_{T}^{2}\right]^{2}}\right] \cos \left(\phi-\phi_{S}\right) \\
& =\frac{1}{8 \pi \boldsymbol{S}_{T}^{2}}\left(\boldsymbol{p}_{T} \cdot \boldsymbol{S}_{T}\right) \frac{2 L_{a}^{2}\left(\Lambda_{a}^{2}\right)+\boldsymbol{p}_{T}^{2}}{L_{a}^{4}\left(\Lambda_{a}^{2}\right)\left[L_{a}^{2}\left(\Lambda_{a}^{2}\right)+\boldsymbol{p}_{T}^{2}\right]^{2}}, \\
& \mathcal{I}_{7}^{\prime d i p}=\int \frac{d \boldsymbol{l}_{T}^{\prime}}{(2 \pi)^{2}} \frac{\left[\left(\boldsymbol{l}_{T}^{\prime}+\boldsymbol{p}_{T}\right) \cdot \boldsymbol{S}_{T}\right]^{2}}{\boldsymbol{S}_{T}^{2}} \frac{1}{\left(\boldsymbol{l}_{T}^{\prime}+\boldsymbol{p}_{T}\right)^{2}\left[\boldsymbol{l}_{T}^{\prime 2}+L_{a}^{2}\left(\Lambda_{a}^{2}\right)\right]^{3}} \\
& =\int_{0}^{\infty} \frac{d\left|\boldsymbol{l}_{T}^{\prime}\right|\left|\boldsymbol{l}_{T}^{\prime}\right|}{(2 \pi)^{2} \boldsymbol{S}_{T}^{2}} \frac{1}{\left[\boldsymbol{l}_{T}^{\prime 2}+L_{a}^{2}\left(\Lambda_{a}^{2}\right)\right]^{3}} \int_{0}^{2 \pi} d \theta \frac{\left[\left|\boldsymbol{l}_{T}^{\prime}\right|\left|\boldsymbol{S}_{T}\right| \cos \left[\theta+\left(\phi-\phi_{S}\right)\right]+\left|\boldsymbol{p}_{T}\right|\left|\boldsymbol{S}_{T}\right| \cos \left(\phi-\phi_{S}\right)\right]^{2}}{\boldsymbol{l}_{T}^{\prime 2}+\boldsymbol{p}_{T}^{2}+2\left|\boldsymbol{l}_{T}^{\prime}\right|\left|\boldsymbol{p}_{T}\right| \cos \theta} \\
& =\frac{1}{(2 \pi)^{2} \boldsymbol{S}_{T}^{2}}\left\{\int_{L_{a}^{2}\left(\Lambda_{a}^{2}\right)}^{L_{a}^{2}\left(\Lambda_{a}^{2}\right)+\boldsymbol{p}_{T}^{2}} \frac{d y}{2 y^{3}} \pi \frac{\boldsymbol{S}_{T}^{2}}{\boldsymbol{p}_{T}^{2}}\left[\boldsymbol{p}_{T}^{2}+\left(\boldsymbol{p}_{T}^{2}+L_{a}^{2}\left(\Lambda_{a}^{2}\right)-y\right) \cos 2\left(\phi-\phi_{S}\right)\right]+\pi \boldsymbol{S}_{T}^{2} \int_{L_{a}^{2}\left(\Lambda_{a}^{2}\right)+\boldsymbol{p}_{T}^{2}}^{\infty} \frac{d y}{2 y^{3}}\right\} \\
& =\frac{1}{(2 \pi)^{2} \boldsymbol{S}_{T}^{2}}\left\{\left[\pi \frac{\boldsymbol{S}_{T}^{2} \boldsymbol{p}_{T}^{2}}{4 L_{a}^{4}\left(\Lambda_{a}^{2}\right)\left[L_{a}^{2}\left(\Lambda_{a}^{2}\right)+\boldsymbol{p}_{T}^{2}\right]^{2}}\left(\left(\cos 2\left(\phi-\phi_{S}\right)+1\right) \boldsymbol{p}_{T}^{2}+\left(\cos 2\left(\phi-\phi_{S}\right)+2\right) L_{a}^{2}\left(\Lambda_{a}^{2}\right)\right)\right]\right. \\
& \left.+\left[\pi \frac{\boldsymbol{S}_{T}^{2}}{4\left[L_{a}^{2}\left(\Lambda_{a}^{2}\right)+\boldsymbol{p}_{T}^{2}\right]^{2}}\right]\right\}
\end{aligned}
$$




$$
\begin{aligned}
& =\frac{1}{16 \pi L_{a}^{4}\left(\Lambda_{a}^{2}\right)\left[L_{a}^{2}\left(\Lambda_{a}^{2}\right)+\boldsymbol{p}_{T}^{2}\right]}\left[L_{a}^{2}\left(\Lambda_{a}^{2}\right)+\boldsymbol{p}_{T}^{2}\left(1+\cos 2\left(\phi-\phi_{S}\right)\right)\right] \\
& =\frac{1}{16 \pi L_{a}^{4}\left(\Lambda_{a}^{2}\right)\left[L_{a}^{2}\left(\Lambda_{a}^{2}\right)+\boldsymbol{p}_{T}^{2}\right]}\left[L_{a}^{2}\left(\Lambda_{a}^{2}\right)+2 \frac{\left(\boldsymbol{p}_{T} \cdot \boldsymbol{S}_{T}\right)^{2}}{\boldsymbol{S}_{T}^{2}}\right]
\end{aligned}
$$

$$
\begin{aligned}
& \mathcal{I}_{2}^{\exp }=\int \frac{d \boldsymbol{l}_{T}^{\prime}}{(2 \pi)^{2}} \frac{e^{-\left[\boldsymbol{l}_{T}^{\prime 2}+L_{a}^{2}\left(m^{2}\right)\right] /\left[(1-x) \Lambda_{a}^{2}\right]}}{\left(\boldsymbol{l}_{T}^{\prime}+\boldsymbol{p}_{T}\right)^{2}\left[\boldsymbol{l}_{T}^{\prime 2}+L_{a}^{2}\left(m^{2}\right)\right]}\left(\boldsymbol{l}_{T}+\boldsymbol{p}_{T}\right)^{2} \\
& =\int_{0}^{\infty} \frac{d\left|\boldsymbol{l}_{T}^{\prime}\right|\left|\boldsymbol{l}_{T}^{\prime}\right|}{(2 \pi)} \frac{e^{-\left[\boldsymbol{l}_{T}^{\prime 2}+L_{a}^{2}\left(m^{2}\right)\right] /\left[(1-x) \Lambda_{a}^{2}\right]}}{\left[\boldsymbol{l}_{T}^{\prime 2}+L_{a}^{2}\left(m^{2}\right)\right]} \\
& =\frac{1}{4 \pi} \int_{L_{a}^{2}\left(m^{2}\right)}^{\infty} d y \frac{e^{-y /\left[(1-x) \Lambda_{a}^{2}\right]}}{y}=\frac{1}{4 \pi} \Gamma\left(0, \frac{L_{a}^{2}\left(m^{2}\right)}{(1-x) \Lambda_{a}^{2}}\right) \text {, } \\
& \mathcal{I}_{3}^{\exp }=\int \frac{d \boldsymbol{l}_{T}^{\prime}}{(2 \pi)^{2}} \frac{e^{-\left[\boldsymbol{l}_{T}^{\prime 2}+L_{a}^{2}\left(m^{2}\right)\right] /\left[(1-x) \Lambda_{a}^{2}\right]}}{\left(\boldsymbol{l}_{T}^{\prime}+\boldsymbol{p}_{T}\right)^{2}\left[\boldsymbol{l}_{T}^{\prime 2}+L_{a}^{2}\left(m^{2}\right)\right]} \frac{\left[\left(\boldsymbol{l}_{T}^{\prime}+\boldsymbol{p}_{T}\right) \cdot \boldsymbol{p}_{T}\right]^{2}}{\boldsymbol{p}_{T}^{2}} \\
& =\int_{0}^{\infty} \frac{d\left|\boldsymbol{l}_{T}^{\prime}\right|\left|\boldsymbol{l}_{T}^{\prime}\right|}{(2 \pi)^{2} \boldsymbol{p}_{T}^{2}} \frac{e^{-\left[\boldsymbol{l}_{T}^{\prime 2}+L_{a}^{2}\left(m^{2}\right)\right] /\left[(1-x) \Lambda_{a}^{2}\right]}}{\left[\boldsymbol{l}_{T}^{\prime 2}+L_{a}^{2}\left(m^{2}\right)\right]} \int_{0}^{2 \pi} d \theta \frac{\left[\left|\boldsymbol{l}_{T}^{\prime}\right|\left|\boldsymbol{p}_{T}\right| \cos \theta+\boldsymbol{p}_{T}^{2}\right]^{2}}{\boldsymbol{l}_{T}^{\prime 2}+\boldsymbol{p}_{T}^{2}+2\left|\boldsymbol{l}_{T}^{\prime}\right|\left|\boldsymbol{p}_{T}\right| \cos \theta} \\
& =\frac{1}{4 \pi \boldsymbol{p}_{T}^{2}}\left\{\int_{0}^{\left|\boldsymbol{p}_{T}\right|} \frac{d\left|\boldsymbol{l}_{T}^{\prime}\right|\left|\boldsymbol{l}_{T}^{\prime}\right|}{\left[\boldsymbol{l}_{T}^{\prime 2}+L_{a}^{2}\left(m^{2}\right)\right]}\left(2 \boldsymbol{p}_{T}^{2}-\boldsymbol{l}_{T}^{\prime 2}\right) e^{-\left[\boldsymbol{l}_{T}^{\prime 2}+L_{a}^{2}\left(m^{2}\right)\right] /\left[(1-x) \Lambda_{a}^{2}\right]}\right. \\
& \left.+\boldsymbol{p}_{T}^{2} \int_{\left|\boldsymbol{p}_{T}\right|}^{\infty} \frac{d\left|\boldsymbol{l}_{T}^{\prime}\right|\left|\boldsymbol{l}_{T}^{\prime}\right|}{\left[\boldsymbol{l}_{T}^{\prime 2}+L_{a}^{2}\left(m^{2}\right)\right]} e^{-\left[\boldsymbol{l}_{T}^{\prime 2}+L_{a}^{2}\left(m^{2}\right)\right] /\left[(1-x) \Lambda_{a}^{2}\right]}\right\} \\
& =\frac{1}{8 \pi \boldsymbol{p}_{T}^{2}}\left\{\int_{L_{a}^{2}\left(m^{2}\right)}^{L_{a}^{2}\left(m^{2}\right)+\boldsymbol{p}_{T}^{2}} d y \frac{2 \boldsymbol{p}_{T}^{2}+L_{a}^{2}\left(m^{2}\right)-y}{y} e^{-y /\left[(1-x) \Lambda_{a}^{2}\right]}+\boldsymbol{p}_{T}^{2} \int_{L_{a}^{2}\left(m^{2}\right)+\boldsymbol{p}_{T}^{2}}^{\infty} d y \frac{e^{-y /\left[(1-x) \Lambda_{a}^{2}\right]}}{y}\right\} \\
& =\frac{1}{8 \pi \boldsymbol{p}_{T}^{2}}\left\{\left[L_{a}^{2}\left(m^{2}\right)+2 \boldsymbol{p}_{T}^{2}\right] \Gamma\left(0, \frac{L_{a}^{2}\left(m^{2}\right)}{(1-x) \Lambda_{a}^{2}}\right)-\left[L_{a}^{2}\left(m^{2}\right)+\boldsymbol{p}_{T}^{2}\right] \Gamma\left(0, \frac{L_{a}^{2}\left(m^{2}\right)+\boldsymbol{p}_{T}^{2}}{(1-x) \Lambda_{a}^{2}}\right)\right. \\
& \left.+(1-x) \Lambda_{a}^{2}\left(e^{-\left[L_{a}^{2}\left(m^{2}\right)+\boldsymbol{p}_{T}^{2}\right] /\left[(1-x) \Lambda_{a}^{2}\right]}-e^{-L_{a}^{2}\left(m^{2}\right) /\left[(1-x) \Lambda_{a}^{2}\right]}\right)\right\}, \\
& \mathcal{I}_{4}^{\exp }=\int \frac{d \boldsymbol{l}_{T}^{\prime}}{(2 \pi)^{2}} \frac{e^{-\left[\boldsymbol{l}_{T}^{\prime 2}+L_{a}^{2}\left(m^{2}\right)\right] /\left[(1-x) \Lambda_{a}^{2}\right]}}{\left(\boldsymbol{l}_{T}^{\prime}+\boldsymbol{p}_{T}\right)^{2}\left[\boldsymbol{l}_{T}^{\prime 2}+L_{a}^{2}\left(m^{2}\right)\right]} \frac{\left[\left(\boldsymbol{l}_{T}^{\prime}+\boldsymbol{p}_{T}\right) \cdot \boldsymbol{p}_{T}\right]^{2}}{\boldsymbol{p}_{T}^{2}}\left(\boldsymbol{l}_{T}^{\prime}+\boldsymbol{p}_{T}\right)^{2} \\
& =\int_{0}^{\infty} \frac{d\left|\boldsymbol{l}_{T}^{\prime}\right|\left|\boldsymbol{l}_{T}^{\prime}\right|}{(2 \pi)^{2} \boldsymbol{p}_{T}^{2}} \frac{e^{-\left[\boldsymbol{l}_{T}^{\prime 2}+L_{a}^{2}\left(m^{2}\right)\right] /\left[(1-x) \Lambda_{a}^{2}\right]}}{\left[\boldsymbol{l}_{T}^{\prime 2}+L_{a}^{2}\left(m^{2}\right)\right]} \int_{0}^{2 \pi} d \theta\left(\left|\boldsymbol{l}_{T}^{\prime}\right|\left|\boldsymbol{p}_{T}\right| \cos \theta+\boldsymbol{p}_{T}^{2}\right) \\
& =\int_{0}^{\infty} \frac{d\left|\boldsymbol{l}_{T}^{\prime}\right|\left|\boldsymbol{l}_{T}^{\prime}\right|}{2 \pi} \frac{e^{-\left[\boldsymbol{l}_{T}^{\prime 2}+L_{a}^{2}\left(m^{2}\right)\right] /\left[(1-x) \Lambda_{a}^{2}\right]}}{\left[\boldsymbol{l}_{T}^{\prime 2}+L_{a}^{2}\left(m^{2}\right)\right]} \\
& =\frac{1}{4 \pi} \int_{L_{a}^{2}\left(m^{2}\right)}^{\infty} d y \frac{e^{-y /\left[(1-x) \Lambda_{a}^{2}\right]}}{y}=\frac{1}{4 \pi} \Gamma\left(0, \frac{L_{a}^{2}\left(m^{2}\right)}{(1-x) \Lambda_{a}^{2}}\right) \equiv \mathcal{I}_{2}^{e x p}, \\
& \mathcal{I}_{5}^{\exp }=\int \frac{d \boldsymbol{l}_{T}^{\prime}}{(2 \pi)^{2}} \frac{e^{-\left[\boldsymbol{l}_{T}^{\prime 2}+L_{a}^{2}\left(m^{2}\right)\right] /\left[(1-x) \Lambda_{a}^{2}\right]}}{\left(\boldsymbol{l}_{T}^{\prime}+\boldsymbol{p}_{T}\right)^{2}\left[\boldsymbol{l}_{T}^{\prime 2}+L_{a}^{2}\left(m^{2}\right)\right]}\left(\boldsymbol{l}_{T}^{\prime}+\boldsymbol{p}_{T}\right)^{4} \\
& =\int_{0}^{\infty} \frac{d\left|\boldsymbol{l}_{T}^{\prime}\right|\left|\boldsymbol{l}_{T}^{\prime}\right|}{(2 \pi)^{2}} \frac{e^{-\left[\boldsymbol{l}_{T}^{\prime 2}+L_{a}^{2}\left(m^{2}\right)\right] /\left[(1-x) \Lambda_{a}^{2}\right]}}{\left[\boldsymbol{l}_{T}^{\prime 2}+L_{a}^{2}\left(m^{2}\right)\right]} \int_{0}^{2 \pi} d \theta\left(\boldsymbol{l}_{T}^{\prime 2}+\boldsymbol{p}_{T}^{2}+2\left|\boldsymbol{l}_{T}^{\prime}\right|\left|\boldsymbol{p}_{T}\right| \cos \theta\right) \\
& =\int_{0}^{\infty} \frac{d\left|\boldsymbol{l}_{T}^{\prime}\right|\left|\boldsymbol{l}_{T}^{\prime}\right|}{2 \pi} \frac{e^{-\left[\boldsymbol{l}_{T}^{\prime 2}+L_{a}^{2}\left(m^{2}\right)\right] /\left[(1-x) \Lambda_{a}^{2}\right]}}{\left[\boldsymbol{l}_{T}^{\prime 2}+L_{a}^{2}\left(m^{2}\right)\right]}\left(\boldsymbol{l}_{T}^{\prime 2}+\boldsymbol{p}_{T}^{2}\right) \\
& =\frac{1}{4 \pi} \int_{L_{a}^{2}\left(m^{2}\right)}^{\infty} d y \frac{y-L_{a}^{2}\left(m^{2}\right)+\boldsymbol{p}_{T}^{2}}{y^{3}} e^{-y /\left[(1-x) \Lambda_{a}^{2}\right]}
\end{aligned}
$$




$$
\begin{aligned}
& =\frac{1}{4 \pi}\left\{\left[\boldsymbol{p}_{T}^{2}-L_{a}^{2}\left(m^{2}\right)\right] \Gamma\left(0, \frac{L_{a}^{2}\left(m^{2}\right)}{(1-x) \Lambda_{a}^{2}}\right)+(1-x) \Lambda_{a}^{2} e^{-L_{a}^{2}\left(m^{2}\right) /\left[(1-x) \Lambda_{a}^{2}\right]}\right\}, \\
& \mathcal{I}_{6}^{e x p}=\int \frac{d \boldsymbol{l}_{T}^{\prime}}{(2 \pi)^{2}} \frac{e^{-\left[\boldsymbol{l}_{T}^{\prime 2}+L_{a}^{2}\left(m^{2}\right)\right] /\left[(1-x) \Lambda_{a}^{2}\right]}}{\left(\boldsymbol{l}_{T}^{\prime}+\boldsymbol{p}_{T}\right)^{2}\left[\boldsymbol{l}_{T}^{\prime 2}+L_{a}^{2}\left(m^{2}\right)\right]} \frac{\left(\boldsymbol{l}_{T}^{\prime}+\boldsymbol{p}_{T}\right) \cdot \boldsymbol{S}_{T}}{\boldsymbol{S}_{T}^{2}} \\
& =\int_{0}^{\infty} \frac{d\left|\boldsymbol{l}_{T}^{\prime}\right|\left|\boldsymbol{l}_{T}^{\prime}\right|}{(2 \pi)^{2} \boldsymbol{S}_{T}^{2}} \frac{e^{-\left[\boldsymbol{l}_{T}^{\prime 2}+L_{a}^{2}\left(m^{2}\right)\right] /\left[(1-x) \Lambda_{a}^{2}\right]}}{\left[\boldsymbol{l}_{T}^{\prime 2}+L_{a}^{2}\left(m^{2}\right)\right]} \int_{0}^{2 \pi} d \theta \frac{\left|\boldsymbol{l}_{T}^{\prime}\right|\left|\boldsymbol{S}_{T}\right| \cos \left(\theta+\phi-\phi_{S}\right)+\left|\boldsymbol{p}_{T}\right|\left|\boldsymbol{S}_{T}\right| \cos \left(\phi-\phi_{S}\right)}{\boldsymbol{l}_{T}^{\prime 2}+\boldsymbol{p}_{T}^{2}+2\left|\boldsymbol{l}_{T}^{\prime}\right|\left|\boldsymbol{p}_{T}\right| \cos \theta} \\
& =\frac{\left|\boldsymbol{p}_{T}\right|\left|\boldsymbol{S}_{T}\right| \cos \left(\phi-\phi_{S}\right)}{4 \pi \boldsymbol{S}_{T}^{2} \boldsymbol{p}_{T}^{2}} \int_{L_{a}^{2}\left(m^{2}\right)}^{L_{a}^{2}\left(m^{2}\right)+\boldsymbol{p}_{T}^{2}} d y \frac{e^{-y /\left[(1-x) \Lambda_{a}^{2}\right]}}{y} \\
& =\frac{\boldsymbol{p}_{T} \cdot \boldsymbol{S}_{T}}{4 \pi \boldsymbol{S}_{T}^{2} \boldsymbol{p}_{T}^{2}}\left\{\Gamma\left(0, \frac{L_{a}^{2}\left(m^{2}\right)}{(1-x) \Lambda_{a}^{2}}\right)-\Gamma\left(0, \frac{L_{a}^{2}\left(m^{2}\right)+\boldsymbol{p}_{T}^{2}}{(1-x) \Lambda_{a}^{2}}\right)\right\}, \\
& \mathcal{I}_{7}^{e x p}=\int \frac{d \boldsymbol{l}_{T}^{\prime}}{(2 \pi)^{2}} \frac{e^{-\left[\boldsymbol{l}_{T}^{\prime 2}+L_{a}^{2}\left(m^{2}\right)\right] /\left[(1-x) \Lambda_{a}^{2}\right]}}{\left(\boldsymbol{l}_{T}^{\prime}+\boldsymbol{p}_{T}\right)^{2}\left[\boldsymbol{l}_{T}^{\prime 2}+L_{a}^{2}\left(m^{2}\right)\right]} \frac{\left[\left(\boldsymbol{l}_{T}^{\prime}+\boldsymbol{p}_{T}\right) \cdot \boldsymbol{S}_{T}\right]^{2}}{\boldsymbol{S}_{T}^{2}} \\
& =\int_{0}^{\infty} \frac{d\left|\boldsymbol{l}_{T}^{\prime}\right|\left|\boldsymbol{l}_{T}^{\prime}\right|}{(2 \pi)^{2} \boldsymbol{S}_{T}^{2}} \frac{e^{-\left[\boldsymbol{l}_{T}^{\prime 2}+L_{a}^{2}\left(m^{2}\right)\right] /\left[(1-x) \Lambda_{a}^{2}\right]}}{\left[\boldsymbol{l}_{T}^{\prime 2}+L_{a}^{2}\left(m^{2}\right)\right]} \int_{0}^{2 \pi} d \theta \frac{\left|\boldsymbol{l}_{T}^{\prime}\right|\left|\boldsymbol{S}_{T}\right| \cos \left(\theta+\phi-\phi_{S}\right)+\left|\boldsymbol{p}_{T}\right|\left|\boldsymbol{S}_{T}\right| \cos \left(\phi-\phi_{S}\right)}{\boldsymbol{l}_{T}^{\prime 2}+\boldsymbol{p}_{T}^{2}+2\left|\boldsymbol{l}_{T}^{\prime}\right|\left|\boldsymbol{p}_{T}\right| \cos \theta} \\
& =\frac{1}{(2 \pi)^{2} \boldsymbol{S}_{T}^{2}}\left\{\int_{L_{a}^{2}\left(m^{2}\right)}^{L_{a}^{2}\left(m^{2}\right)+\boldsymbol{p}_{T}^{2}} d y \frac{e^{-y /\left[(1-x) \Lambda_{a}^{2}\right]}}{y} \pi \frac{\boldsymbol{S}_{T}^{2}}{\boldsymbol{p}_{T}^{2}}\left[\boldsymbol{p}_{T}^{2}+\left(\boldsymbol{p}_{T}^{2}+L_{a}^{2}\left(m^{2}\right)-y\right) \cos 2\left(\phi-\phi_{S}\right)\right]\right. \\
& \left.+\pi \boldsymbol{S}_{T}^{2} \int_{L_{a}^{2}\left(m^{2}\right)+p_{T}^{2}}^{\infty} d y \frac{e^{-y /\left[(1-x) \Lambda_{a}^{2}\right]}}{y}\right\} \\
& =\frac{1}{8 \pi \boldsymbol{p}_{T}^{2}}\left\{(1-x) \Lambda_{a}^{2}\left(e^{-\left[L_{a}^{2}\left(m^{2}\right)+p_{T}^{2}\right] /\left[(1-x) \Lambda_{a}^{2}\right]}-e^{-L_{a}^{2}\left(m^{2}\right) /\left[(1-x) \Lambda_{a}^{2}\right]}\right) \cos 2\left(\phi-\phi_{S}\right)\right. \\
& +\Gamma\left(0, \frac{L_{a}^{2}\left(m^{2}\right)}{(1-x) \Lambda_{a}^{2}}\right)\left[\left[\boldsymbol{p}_{T}^{2}+L_{a}^{2}\left(m^{2}\right)\right] \cos 2\left(\phi-\phi_{S}\right)+\boldsymbol{p}_{T}^{2}\right] \\
& \left.-\Gamma\left(0, \frac{L_{a}^{2}\left(m^{2}\right)+\boldsymbol{p}_{T}^{2}}{(1-x) \Lambda_{a}^{2}}\right)\left[\boldsymbol{p}_{T}^{2}+L_{a}^{2}\left(m^{2}\right)\right] \cos 2\left(\phi-\phi_{S}\right)\right\} \text {. }
\end{aligned}
$$

[1] J. C. Collins and D. E. Soper, Nucl. Phys. B194, 445 (1982).

[2] M. Burkardt, Phys. Rev. D62, 071503 (2000), hep-ph/0005108.

[3] M. Diehl, Eur. Phys. J. C25, 223 (2002), hep-ph/0205208.

[4] X. Ji, Phys. Rev. Lett. 91, 062001 (2003), hep-ph/0304037.

[5] A. V. Belitsky, X. Ji, and F. Yuan, Phys. Rev. D69, 074014 (2004), hep-ph/0307383.

[6] S. Boffi and B. Pasquini, Riv. Nuovo Cim. 30, 387 (2007), 0711.2625.

[7] X. Ji, J.-P. Ma, and F. Yuan, Phys. Rev. D71, 034005 (2005), hep-ph/0404183.

[8] X. Ji, J.-P. Ma, and F. Yuan, Phys. Lett. B597, 299 (2004), hep-ph/0405085.

[9] A. Bacchetta, M. Boglione, A. Henneman, and P. J. Mulders, Phys. Rev. Lett. 85, 712 (2000), hep-ph/9912490.

[10] P. V. Pobylitsa, (2003), hep-ph/0301236.

[11] S. J. Brodsky and F. Yuan, Phys. Rev. D74, 094018 (2006), hep-ph/0610236.

[12] A. Bacchetta, D. Boer, M. Diehl, and P. J. Mulders, (2008), 0803.0227.

[13] S. J. Brodsky, D. S. Hwang, and I. Schmidt, Phys. Lett. B530, 99 (2002), hep-ph/0201296.

[14] D. Boer and P. J. Mulders, Phys. Rev. D57, 5780 (1998), hep-ph/9711485.

[15] A. Bacchetta et al., JHEP 02, 093 (2007), hep-ph/0611265.

[16] J. C. Collins, Phys. Lett. B536, 43 (2002), hep-ph/0204004.

[17] U. D'Alesio and F. Murgia, (2007), arXiv:0712.4328 [hep-ph].

[18] U. D'Alesio and F. Murgia, Phys. Rev. D70, 074009 (2004), hep-ph/0408092.

[19] D. W. Sivers, Phys. Rev. D41, 83 (1990).

[20] M. Anselmino et al., (2005), hep-ph/0511017.

[21] D. Boer and P. J. Mulders, Phys. Rev. D57, 5780 (1998), hep-ph/9711485. 
[22] B. Zhang, Z. Lu, B.-Q. Ma, and I. Schmidt, Phys. Rev. D77, 054011 (2008), arXiv:0803.1692 [hep-ph].

[23] S. J. Brodsky, D. S. Hwang, and I. Schmidt, Nucl. Phys. B642, 344 (2002), hep-ph/0206259.

[24] M. Burkardt and D. S. Hwang, Phys. Rev. D69, 074032 (2004), hep-ph/0309072.

[25] K. Goeke, S. Meissner, A. Metz, and M. Schlegel, Phys. Lett. B637, 241 (2006), hep-ph/0601133.

[26] Z. Lu and I. Schmidt, Phys. Rev. D75, 073008 (2007), hep-ph/0611158.

[27] S. Meissner, A. Metz, and K. Goeke, Phys. Rev. D76, 034002 (2007), hep-ph/0703176.

[28] J.-W. Qiu, W. Vogelsang, and F. Yuan, Phys. Rev. D76, 074029 (2007), arXiv:0706.1196 [hep-ph].

[29] M. Burkardt and B. Hannafious, Phys. Lett. B658, 130 (2008), arXiv:0705.1573 [hep-ph].

[30] L. P. Gamberg, G. R. Goldstein, and K. A. Oganessyan, Phys. Rev. D67, 071504 (2003), hep-ph/0301018.

[31] L. P. Gamberg, G. R. Goldstein, and K. A. Oganessyan, Phys. Rev. D68, 051501(R) (2003), hep-ph/0307139.

[32] Z. Lu and B.-Q. Ma, Nucl. Phys. A741, 200 (2004), hep-ph/0406171.

[33] L. P. Gamberg and G. R. Goldstein, Phys. Lett. B650, 362 (2007), hep-ph/0506127.

[34] V. Barone, Z. Lu, and B.-Q. Ma, Eur. Phys. J. C49, 967 (2007), hep-ph/0612350.

[35] Z. Lu, B.-Q. Ma, and I. Schmidt, Phys. Rev. D75, 094012 (2007), arXiv:0704.2292 [hep-ph].

[36] L. P. Gamberg, G. R. Goldstein, and M. Schlegel, Phys. Rev. D77, 094016 (2008), 0708.0324.

[37] A. Bianconi and M. Radici, Phys. Rev. D71, 074014 (2005), hep-ph/0412368.

[38] A. Bianconi and M. Radici, Phys. Rev. D72, 074013 (2005), hep-ph/0504261.

[39] A. Bianconi and M. Radici, Phys. Rev. D73, 034018 (2006), hep-ph/0512091.

[40] A. Bianconi and M. Radici, Phys. Rev. D73, 114002 (2006), hep-ph/0602103.

[41] M. Radici, F. Conti, A. Bacchetta, and A. Bianconi, (2007), arXiv:0708.0232 [hep-ph].

[42] R. Jakob, P. J. Mulders, and J. Rodrigues, Nucl. Phys. A626, 937 (1997), hep-ph/9704335.

[43] B. Pasquini, S. Cazzaniga, and S. Boffi, (2008), 0806.2298.

[44] D. Boer, S. J. Brodsky, and D. S. Hwang, Phys. Rev. D67, 054003 (2003), hep-ph/0211110.

[45] G. R. Goldstein and L. Gamberg, (2002), hep-ph/0209085.

[46] A. Bacchetta, A. Schäfer, and J.-J. Yang, Phys. Lett. B578, 109 (2004), hep-ph/0309246.

[47] F. Yuan, Phys. Lett. B575, 45 (2003), hep-ph/0308157.

[48] I. O. Cherednikov, U. D'Alesio, N. I. Kochelev, and F. Murgia, Phys. Lett. B642, 39 (2006), hep-ph/0606238.

[49] A. Courtoy, F. Fratini, S. Scopetta, and V. Vento, (2008), 0801.4347.

[50] Z. Lu and B.-Q. Ma, Phys. Rev. D70, 094044 (2004), hep-ph/0411043.

[51] S. J. Brodsky, D. S. Hwang, B.-Q. Ma, and I. Schmidt, Nucl. Phys. B593, 311 (2001), hep-th/0003082.

[52] G. P. Lepage and S. J. Brodsky, Phys. Rev. D22, 2157 (1980).

[53] V. Barone and P. G. Ratcliffe, Transverse Spin Physics (World Scientific, River Edge, USA, 2003).

[54] H. Avakian, A. V. Efremov, P. Schweitzer, and F. Yuan, (2008), 0805.3355.

[55] D. Boer, P. J. Mulders, and F. Pijlman, Nucl. Phys. B667, 201 (2003), hep-ph/0303034.

[56] J. C. Collins and D. E. Soper, Nucl. Phys. B193, 381 (1981).

[57] J. A. Robinson and T. G. Rizzo, Phys. Rev. D33, 2608 (1986).

[58] M. E. Peskin and D. V. Schroeder, An introduction to quantum field theory (Addison-Wesley, Reading, MA, USA, 1995).

[59] R. E. Cutkosky, J. Math. Phys. 1, 429 (1960).

[60] A. Kotzinian, (2008), 0806.3804.

[61] M. Burkardt, Phys. Rev. D66, 114005 (2002), hep-ph/0209179.

[62] A. Bacchetta, U. D'Alesio, M. Diehl, and C. A. Miller, Phys. Rev. D70, 117504 (2004), hep-ph/0410050.

[63] ZEUS, S. Chekanov et al., Phys. Rev. D67, 012007 (2003), hep-ex/0208023.

[64] M. Glück, E. Reya, M. Stratmann, and W. Vogelsang, Phys. Rev. D63, 094005 (2001), hep-ph/0011215.

[65] Asymmetry Analysis, M. Hirai, S. Kumano, and N. Saito, Phys. Rev. D69, 054021 (2004), hep-ph/0312112.

[66] I. C. Cloet, W. Bentz, and A. W. Thomas, Phys. Lett. B659, 214 (2008), 0708.3246.

[67] M. Burkardt, Phys. Rev. D69, 091501 (2004), hep-ph/0402014.

[68] H. Avakian, S. J. Brodsky, A. Deur, and F. Yuan, Phys. Rev. Lett. 99, 082001 (2007), 0705.1553.

[69] H. Mkrtchyan et al., (2007), 0709.3020.

[70] E. Leader, A. V. Sidorov, and D. B. Stamenov, Int. J. Mod. Phys. A13, 5573 (1998), hep-ph/9708335.

[71] M. Hirai, S. Kumano, and M. Miyama, Comput. Phys. Commun. 111, 150 (1998), hep-ph/9712410.

[72] M. Anselmino et al., Phys. Rev. D75, 054032 (2007), hep-ph/0701006.

[73] HERMES, A. Airapetian et al., Phys. Rev. Lett. 94, 012002 (2005), hep-ex/0408013.

[74] COMPASS, E. S. Ageev et al., Nucl. Phys. B765, 31 (2007), hep-ex/0610068.

[75] M. Anselmino et al., (2008), 0807.0173.

[76] V. Barone, A. Drago, and P. G. Ratcliffe, Phys. Rept. 359, 1 (2002), hep-ph/0104283.

[77] B. Pasquini, M. Pincetti, and S. Boffi, Phys. Rev. D72, 094029 (2005), hep-ph/0510376.

[78] M. Wakamatsu, Phys. Lett. B653, 398 (2007), 0705.2917.

[79] M. Anselmino et al., (2008), 0805.2677.

[80] J. C. Collins et al., (2005), hep-ph/0510342.

[81] QCDSF, M. Gockeler et al., Phys. Rev. Lett. 98, 222001 (2007), hep-lat/0612032. 